DESY $95-238$

ISSN $0418-9833$

December 1995

\title{
Consistent Histories and Operational Quantum Theory
}

\author{
Oliver Rudolph* \\ II. Institut für Theoretische Physik, Universität Hamburg \\ Luruper Chaussee 149 \\ D-22761 Hamburg, Germany
}

\begin{abstract}
In this work a generalization of the consistent histories approach to quantum mechanics is presented. We first critically review the consistent histories approach to nonrelativistic quantum mechanics in a mathematically rigorous way and give some general comments about it. We investigate to what extent the consistent histories scheme is compatible with the results of the operational formulation of quantum mechanics. According to the operational approach nonrelativistic quantum mechanics is most generally formulated in terms of effects, states and operations. We formulate a generalized consistent histories theory using the concepts and the terminology which have proven useful in the operational formulation of quantum mechanics. The logical rule of the logical interpretation of quantum mechanics is generalized to the present context. The algebraic structure of the generalized theory is studied in detail.
\end{abstract}

\footnotetext{
* Internet: rudolph@x4u2.desy.de
} 


\section{Introduction}

This article is the outcome of an attempt to understand the relation of two seemingly completely different modern formulations of quantum mechanics. On the one hand we consider the insightful modern operational reformulation and generalization of quantum mechanics due to many authors. We refer the reader to the monographs by Davies (1976), Kraus (1983), Ludwig (1970, 1972-79, 1983), Busch, Lahti and Mittelstaedt (1991), Busch, Grabowski and Lahti (1995) and references therein. On the other hand, we study the consistent histories approach and particularly Omnès' logical interpretation of quantum mechanics.

The operational approach is based on an analysis of the quantum mechanical measuring process and is formulated in terms of accompanying purely operational concepts: according to the operational approach nonrelativistic quantum mechanics is most generally formulated in terms of effects, states and operations and the accompanying notions like "positive operator valued measures.' The operational approach incorporates many ideas from the older orthodox interpretations (cf. (Jammer, 1974; Primas, 1983; Scheibe, 1973)), in particular the measuring process is considered to be of central importance in the foundations of quantum mechanics.

In contrast, the history approach is the outcome of an effort to deal with quantum mechanics of single closed systems, i.e., systems which do neither interact with their environment nor are exposed to measurements. In this approach the measuring process is not considered to be a fundamental notion but rather a derived one.

In this work we adopt a realistic, individual interpretation of quantum mechanics. Further, we will adopt a somewhat conservative point of view in that we will consider the operational approach as a physically fundamental and meaningful approach in the sense that its results and its range of applications must be contained in (or derivable from) every generalized formulation of quantum mechanics based on a realistic interpretation, in particular in the consistent histories formulation of quantum mechanics. Thus we will investigate whether the history approach to quantum mechanics can be formulated and generalized in terms of the notions and the concepts of the operational formulation of quantum mechanics.

The history approach to nonrelativistic quantum mechanic has received much attention in the last decade. The consistent histories approach to nonrelativistic quantum mechanics has been inaugurated in a seminal paper by Griffiths (1984). Nonrelativistic quantum mechanics in its standard formulation is not a theory which describes dynamical processes in time, but it is a theory which gives probabilities to various possibilities. The history approach to quantum mechanics can be looked upon as an attempt to remedy this situation by introducing time sequences of possibilities as a rough substitute for dynamical processes. Nowadays there are two mainstreams of research in this field. The first one is mainly due to Roland Omnès, who uses histories as a framework for constructing a realistic and individual interpretation of quantum mechanics. The resulting logical interpretation is based on some simple logical rules which we will review below. It is thus fair to say that the logical interpretation is a purely epistemological interpretation. Histories are not necessarily considered to represent features belonging to physical reality and are not considered to be ontological models for the quantum system (most generally the universe) at hand, but are simply considered as a useful tool to talk about 
quantum systems (more precisely, in the logical interpretation histories are thought to represent the only meaningful assertions about a quantum mechanical system in the language of physics).

On the other hand, Gell-Mann and Hartle consider the history approach as a convenient setting to discuss the quantum mechanics of closed systems, i.e., most generally the universe (Gell-Mann and Hartle, 1990a-c, 1993, 1994, 1995; Hartle, 1991, 1994). In this way they hope to circumvent some of the conceptual and mathematical difficulties inherent in quantum cosmology. They propose to use histories to describe the events that have taken place and that will take place in the universe. They pay special attention to what they call quasiclassical domains. [They prefer now the term quasiclassical realm (Gell-Mann and Hartle, 1995).] Loosely speaking a quasiclassical domain is an exhaustive set of mutually exclusive coarse-grained alternative histories which can be ascribed probabilities and whose individual histories are described largely by alternative values of a limited set of quasiclassical variables (at a succession of times) which exhibit patterns of classical correlation in time subject to quantum mechanical fluctuations. In order to make pre- and retrodiction one needs information about the initial state of the universe and about past events. Since the human sensory perceptions are rather limited, we can deal in general only with sets of alternative coarse-grained histories. Furthermore, it is only possible to ascribe probabilities to suitably coarse-grained histories provided some consistency conditions are satisfied. The research in this direction is faced with severe difficulties, as discussed by Dowker and Kent (1996) and Kent (1995). In this work we will not be concerned with the Gell-Mann-Hartle approach.

Griffiths has recently proposed an interesting modification of these two approaches (Griffiths, 1995). Griffiths essentially claims (amongst others) that inconsistent histories can be considered to be 'objectively' true separately even though inconsistent histories cannot be combined, either in constructing descriptions or in making logical inferences about them, i.e., even though assertions involving inconsistent histories are meaningless.

Many of the central concepts of the modern operational formulation are foreign to the standard consistent histories approach and therefore the standard consistent histories approach is considerably and unnecessarily restricted in its range of applications. In the present work we formulate a generalized consistent histories theory using the terminology and the concepts which have proven useful in the operational formulation of quantum mechanics. The starting points of the present work are the fact that the observables in quantum mechanics have to be identified with positive operator valued measures and the reasonable claim that the consistent histories approach should take into account the full set of quantum mechanical observables.

This work is organized as follows. In Section 2 we rewrite the formalism of the standard consistent histories approach to quantum mechanics in full generality in a mathematically precise way. Moreover, this section contains a brief (and inevitably incomplete) introduction to the operational formulation of quantum mechanics based on the notion of effect and the concept of generalized observables. The first part of Section 2 contains a discussion of the interpretation of quantum mechanics underlying our generalized history approach, i.e., we discuss the 
meaning of concepts like 'observable,' 'property,' and the like. The reader should not expect a thorough philosophical analysis. On the contrary, we adopt philosophically somewhat naive but physically pragmatic points of view. In Section 3 some aspects of the logical interpretation of quantum mechanics are discussed. However, the treatment in Section 3 is by no means complete and only the aspect relevant for this work are discussed. An extensive discussion of the logical interpretation can be found in Omnès' original work (Omnès, 1988a-c, 1989, 1990, 1992, 1994, 1995). In Section 4 we describe a generalized history theory, which generalizes the standard consistent histories approach. In Section 4 the algebraic and order structures of our generalized history approach are investigated. The notion of decoherence functional is extended to the generalized framework and some of its elementary properties are discussed. It will turn out that so-called effect algebras or difference posets (D-posets) are the basic algebraic structure in our formulation. We explicitly construct the tensor product of two sets of effects on some Hilbert space in the category of effect algebras. The central result of Section 4.3 is the identification of the consistent sets of generalized histories on which the decoherence functional induces a probability measure and the formulation of the generalized logical rule of interpretation. Finally, in Section 4.4 we use our results to study the possible algebraic structures of more general quantum mechanical history theories not necessarily restricted to nonrelativistic theories. This can be viewed as a generalization of Isham's temporal quantum logic (Isham, 1994). In Section 5 we discuss our results and present our conclusions.

It must be emphasized that the representation and the interpretation of the consistent histories approach in this work might not be accepted by the authors cited. The present work solely reflects the inclination and the views of this author.

\section{Basic Facts about Consistent Histories and Opera- tional Quantum Mechanics}

In this section we first review formal aspects of the consistent histories approach to quantum theory initiated by Griffiths and further developed by Omnès, Gell-Mann and Hartle and Isham and others. This section contains essentially known material. It can be read as a commentary on the standard texts. There is no general agreement in the literature, however, concerning the interpretational issues discussed in this section. Our treatment is based on a realistic and individual interpretation of probabilities in quantum mechanics as outlined, e.g., in (Busch et al., 1995; Omnès, 1992, 1994; Popper, 1982). [The views adopted in the present work differ, however, in some aspects from those in (Busch et al., 1995; Omnès, 1992, 1994; Popper, 1982).] This interpretation has as one of its basic assumptions that there exists a definite physical reality, which exists independently of and changes independently of (human) observers.

Remark 1 Quantum mechanics as a probabilistic theory provides no solution of the problem of the 'actualization of facts.' This is the problem why some events take place in the real world while others do not. In quantum measurement theory this problem occurs as 'objectification problem' (Busch et al., 1991). 
In our interpretation we carefully distinguish between the mathematical formalism of a theory (the syntactical part of a theory) and the semantical part of a theory (the interpretation), which relates some abstract concepts of the mathematical formalism to the objects of physical reality which they represent. Only those parts of the mathematical formalism can be thought of as corresponding to elements of physical reality which are interpreted in the semantical part of the theory.

A physical system is a part of the physical reality which has to be regarded (in at least one respect) as a physical unit. The relevant aspects characterizing a physical system should not be affected by the interaction with other parts of physical reality (at least to a certain degree of accuracy). Every physical theory (in particular quantum mechanics) is concerned with the description (and understanding) of some physical systems. In this work we do not aim to give a precise definition of the notion 'physical system,' but rather adopt an abstract (but pragmatic) point of view and represent a physical system in the mathematical formalism by a collection of observables ('the observables of the system'). Observables are part of the semantical language of physical theories; they represent and systematize in the mathematical formalism possible events which may occur in the physical systems and which can be described by the theory at hand.

There is a longstanding debate in quantum physics which entities in the formalism can be identified with properties of a system and what structure the space of all properties is supposed to have. No agreement has been achieved yet, for some different points of view see, e.g., (Omnès, 1994; Ludwig, 1983; von Neumann, 1932; Giuntini and Greuling, 1989; Cattaneo and Laudisa, 1994). Most authors use the terminology introduced by von Neumann, who has identified the projection operators with the possible 'properties' of the quantum mechanical system (von Neumann, 1932). However, Ludwig (1970, 1983) has stressed that the space of all projection operators does not satisfy some conditions which may intuitively be associated with the notion of 'property.' In contrast, the author of the present work believes that the problem of identifying the properties of a quantum mechanical system is a pseudo-problem. We simply identify the possible properties of a system with the propositions specifying the domain of values for some observable. That is, we view a system as a carrier of properties and as a bearer of dispositions. However, in the sequel we will use the phrase 'proposition about a system' or following partly Birkhoff and von Neumann (1936) the term physical quality for this concept (actually this term has been used in a different sense by Birkhoff and von Neumann) and not the term 'property' and also not the term 'pseudoproperty' coined by Ludwig. What exactly we mean by physical quality and physical property will be specified below. Whether or not the physical qualities and physical properties can be measured ideally or repeatedly (Busch et al., 1995) and whether or not the set of all qualities and the set of all properties satisfies some more or less intuitive axioms as claimed, e.g., by Ludwig, is of no concern to us. The notions of observable, physical quality and even the features which characterize a physical system as such are inferred on theoretical grounds; that is, which features characterize a physical system and what a physical quality is depends upon the theory and upon the interpretation we use. (However, it is clear that the question whether a given theory with its accompanying notions of 
system and physical quality is a 'good' theory cannot be decided on purely theoretical grounds.)

In this work we encounter the following point of view which is essentially due to Griffiths (1995): We assert that for a quantum mechanical system there are several incompatible (or complementary) frameworks for its theoretical description in terms of physical qualities and that there are several incompatible (or complementary) frameworks for making logical inferences about physical qualities or about time sequences of physical qualities. We do not even claim that the possible frameworks for the theoretical description coincide with the possible frameworks for making logical inferences. (As we will see below in our generalized history approach this is nevertheless the case.) All different frameworks (or in Griffiths' terminology topics of conversation) are similarly objective. That is, the symmetrical treatment of several incompatible frameworks in the mathematical formalism of quantum mechanics is not broken in the interpretation and (as is asserted in the interpretation) also not in the physical reality in the following sense: it is the whole objective physical situation (for instance an experimental arrangement) which determines the framework that should be used for the description and reasoning.

Thus we assert that for every physical system there are elements of physical reality which cannot be combined either in constructing a theoretical description or in making logical inferences about them. Such complementary elements of reality are not independent. The exact form of the framework for the theoretical description and for making logical inferences is specified below in Rule 1 for the standard logical interpretation of quantum mechanics and in Rule 2 for the generalized logical interpretation developed in this work.

We consider a quantum mechanical system $\mathcal{S}$ without superselection rules represented by a separable complex Hilbert space $\mathbb{H}$ and a Hamiltonian operator $H$. Every physical state of the considered system is mathematically represented by a density operator on $\mathbb{H}$, i.e., a linear, positive, trace-class operator on $\mathbb{H}$ with trace 1 . We denote the set of all trace-class operators on $\mathbb{H}$ by $\mathcal{T}(\mathbb{H})$ and the set of all density operators on $\mathbb{H}$ by $\mathcal{T}(\mathbb{H})_{1}^{+}$. The time evolution is governed by the unitary operator $U\left(t^{\prime}, t\right)=\exp \left(-i\left(t^{\prime}-t\right) H / \hbar\right)$ which maps states at time $t$ into states at time $t^{\prime}$ and satisfies $U\left(t^{\prime \prime}, t^{\prime}\right) U\left(t^{\prime}, t\right)=U\left(t^{\prime \prime}, t\right)$ and $U(t, t)=1$.

In the familiar formulations of quantum mechanics the observables are identified with (and represented by) the self-adjoint operators on $\mathbb{H}$ and according to the spectral theorem observables can be identified with projection operator valued (PV) measures on the real line; that is, there is a one-to-one correspondence between self-adjoint operators on $\mathbb{H}$ and maps $\mathcal{O}: \mathcal{B}(\mathbb{R}) \rightarrow \mathcal{P}(\mathbb{H})$, such that $\mathcal{O}(\mathbb{R})=1$ and $\mathcal{O}\left(\cup_{i} K_{i}\right)=\sum_{i} \mathcal{O}\left(K_{i}\right)$ for every pairwise disjoint sequence $\left\{K_{i}\right\}_{i}$ in $\mathcal{B}(\mathbb{R})$ (the series converging in the ultraweak topology). Here $\mathcal{B}(\mathbb{R})$ denotes the Borel $\sigma$-algebra of $\mathbb{R}$ and $\mathcal{P}(\mathbb{H})$ denotes the set of projection operators on $\mathbb{H}$, i.e., self-adjoint operators $P$ with $P=P P$. PV measures are also called SPECTRAL MEASURES.

We adopt the following physical interpretation of the so defined observables: all meaningful propositions about the considered system specify that the value of some observable $\mathcal{O}$ lies in some set $B \in \mathcal{B}(\mathbb{R})$. We also say that such a specification of the value of some observable represents a physical quality of the system or a proposition about a system; in one word a proposition 
is a speak-able. All other propositions about the system in the formalism of the theory are considered as representing only syntactical statements. The same idea is often expressed in a modern language by saying that only observables represent the be-ables in the theory and that all other objects in the mathematical formalism have no beable status. We can state our assertion also as follows: beables represent the possible events that may occur in the physical system (Bell, 1987).

Note 1 Beables as defined above are surely the pre-eminent concepts in the theory that may be thought of as corresponding to some elements of reality. We make no attempt to define what the term reality exactly means and we do not claim that beables are the only elements in the formalism which may be considered real. The term beable is, however, introduced to stress that there are several degrees of reality in a physical theory. By way of an example, the possible values of the coordinates of an elementary particle describe possible events in physical reality and have thus beable status in the quantum mechanical description of the particle, whereas, say, the number of degrees of freedom of the same elementary particle is a somewhat abstract characteristic of the particle which has no beable status in the theory but which may nevertheless be considered real. In particular the state of the system has no beable status in the interpretation of quantum mechanics outlined here. The state of a system collects all necessary information of the past history of the system (e.g., preparation procedures) to compute probabilities for future events. In the words of Popper (1982) 'the real state of a physical system, at any moment, may be conceived as the sum total of its dispositions-or its potentialities, or possibilities, or propensities.' Thus we think of the state as an abstract concept in the syntactical part of the theory (i.e., in the mathematical formalism) characterizing the real physical system. To be not misunderstood, states are completely objective; even though we do not think of states as real properties of the physical system, we may nevertheless consider states as properties of the integral physical situation. Since quantum mechanics is a probabilistic theory, Gleason's theorem fixes the notion of state in the formalism of quantum mechanics (see below).

The probability of a physical quality represented by the projection operator $P$ is in the state represented by the density operator $\varrho$ given by $\operatorname{tr}(\varrho P)$, where tr denotes the trace in $\mathbb{H}$.

There is no agreement in the literature concerning the interpretation of this probability. According to a minimal operational interpretation it represents the probability that a physical quality is found in a measurement.

At this point it should be stressed that one has to carefully distinguish between physical qualities and the associated projection operators. Several physical qualities may correspond to the same projection operator. However, from the operational approach to quantum mechanics (Kraus, 1983; Ludwig, 1983) it follows, that different physical qualities corresponding to the same projection operator cannot be distinguished by performing yes-no experiments and thus can only collectively be considered to be true or false. For this reason in standard quantum mechanics the projection operators are said to represent properties of the system.

Remark 2 In this work we adopt Popper's propensity interpretation of probabilities (Popper, 1959/60, 1982). In the propensity interpretation the classes of experimentally indistinguish- 
able physical qualities (represented by some projection operator) represent the "beables" and the probabilities are thought to express the tendencies in the behaviour of the system. They are measures of the propensity or of the tendency of a possibility (i.e., a beable) to realize itself upon repetition. Probabilities are associated with a single system in contrast to the usual frequency interpretation of probabilities where probabilities are numbers characterizing ensembles of (similarly prepared) systems. Nevertheless the probabilistic statements in the theory can be tested by actual sequences of measurements. Popper ascribes the priority for the propensity interpretation to a large extend to Landé (1965).

Note 2 A comprehensive and critical discussion of different possibilities to consistently interpret the term 'probability' can be found in the monographs by K. Popper (1982, 1994). In his discussion of quantum mechanics Popper discusses besides the propensity interpretation of probability various issues regarding the interpretation of quantum mechanics and other related areas of physics. However, although the author of the present work believes that the propensity interpretation of probability is wellsuited for the consistent histories approach to quantum mechanics and for the logical interpretation, the author does not wish to imply that he accepts all of the assertions and claims in (Popper, 1982, 1994). On the contrary, this author believes that Popper's interpretation of quantum mechanics as a whole is physically incomplete and that something like the logical rule of interpretation has to be added to it in order to make it physically complete.

According to standard quantum mechanics physical qualities correspond to projection operators and observables to PV measures. However, as discussed at length by Davies (1976), Kraus (1983) and Busch et al. $(1991,1995)$ there is good reason to generalize the usual notion of an observable in quantum mechanics. These generalized observables have been developed and applied independently in various branches of physics, such as, e.g., stochastic quantum mechanics (Prugověcki, 1992), quantum optics (Davies, 1976) or even the (conceptual) foundations of quantum mechanics (Ludwig, 1983). For a detailed motivation and physical justification for the introduction of generalized observables, we refer in particular to the recent lucid monograph by Busch, Grabowski and Lahti (1995).

Positive and bounded operators $F$ on $\mathbb{H}$, satisfying

$$
0 \leq F \leq 1
$$

are commonly called EFFECTS and the set of all effects on the Hilbert space $\mathbb{H}$ will be denoted by $\mathfrak{E}(\mathbb{H})$. We further denote the set of all bounded, linear operators on $\mathbb{H}$ by $\mathcal{B}(\mathbb{H})$, the set of all positive (and hence Hermitean), linear, bounded operators on $\mathbb{H}$ by $\mathcal{B}(\mathbb{H})^{+}$, and the set of all projection operators on $\mathbb{H}$ by $\mathcal{P}(\mathbb{H})$.

If $\mathbb{H}$ is an infinite dimensional Hilbert space, then the set of all projection operators $\mathcal{P}(\mathbb{H})$ on $\mathbb{H}$ is weakly dense in $\mathfrak{E}(\mathbb{H})$ (Davies, 1976).

Generalized obSERVABLES are now identified with positive operator valued (POV) measures on some measurable space $(\Omega, \mathcal{F})$, i.e., maps $\mathrm{O}: \mathcal{F} \rightarrow \mathfrak{E}(\mathbb{H})$ with the properties: 
- $\mathrm{O}(A) \geq \mathrm{O}(\emptyset)$, for all $A \in \mathcal{F}$;

- Let $\left\{A_{i}\right\}$ be a countable set of disjoint sets in $\mathcal{F}$, then $\mathrm{O}\left(\cup_{i} A_{i}\right)=\sum_{i} \mathrm{O}\left(A_{i}\right)$, the series converging ultraweakly;

- $\mathrm{O}(\Omega)=1$.

Generalized observables are also called EFFECT VALued MEASURES. Ordinary observables (associated with self-adjoint operators on $\mathbb{H}$ ) are then identified with the projection valued measures on the real line $\mathbb{R}$.

Note 3 Notice, that in general there is no unambiguous representation of generalized observables as self-adjoint operators on $\mathbb{H}$. However, there is a one-one correspondence between maximal symmetric operators on $\mathbb{H}$ and POV measures on $\mathbb{H}$ (Busch et al., 1995).

The set $\Omega$ represents the set of all possible values of the observable $O$ and $\mathcal{F}$ represents the allowed coarse-grainings in $\Omega$. Generalized observables which are not ordinary observables are often also called UNSHARP OBSERVABLES.

Note 4 The term unsharp observable is used with different meanings in the literature, see e.g., (Busch et al., 1995). However, the term unsharp is somewhat misleading. Admittedly, some (but not all) generalized observables arise as smeared versions of ordinary observables. In general, however, generalized observables must not be considered as unsharp or limited counterparts of some underlying more sharply defined observables, but rather as independent entities in their own right.

According to Gleason's theorem, for every positive, normalized $\sigma$-additive map $p: \mathfrak{E}(\mathbb{H}) \rightarrow$ $[0,1]$, also called generalized probability measure, there exists a unique $\varrho \in \mathcal{T}(\mathbb{H})_{1}^{+}$, such that $p(F)=\operatorname{tr}(\varrho F)$. (A map $p: \mathfrak{E}(\mathbb{H}) \rightarrow[0,1]$ is called $\sigma$-ADDITIVE if for every countable collection $\left\{E_{i}\right\}$ of elements of $\mathfrak{E}(\mathbb{H})$ such that $\sum_{i} E_{i} \leq 1$, one has $p\left(\sum_{i} E_{i}\right)=\sum_{i} p\left(E_{i}\right)$ (convergence in the weak operator topology).) It also follows from Gleason's theorem that an effect valued measure represents the most general notion of an observable compatible with the probabilistic structure of Hilbert space quantum mechanics. Gleason's theorem has first been proved in (Gleason, 1957); a short proof can for instance be found in (Maeda, 1989).

Generalizing our above terminology, we regard all propositions specifying the value of some (generalized) observable as (generalized) physical qualities . In order to discriminate physical qualities corresponding to ordinary observables from physical qualities corresponding to generalized observables, we will sometimes call the former 'ordinary physical qualities' and the latter 'generalized physical qualities.' In the generalized approach to every physical quality there is associated one effect operator.

Every physical quality represents some dichotomy (or binary alternative or yes-no alternative) at every instant of time. There are physical qualities which are always true, e.g., the physical qualities asserting that the value of some observable lies somewhere (without further specification) in the space of its possible values. Such physical qualities will be pairwise identified and collectively denoted by 1 . Similarly, physical qualities which are always false will 
also be pairwise identified and collectively denoted by 0 . We will denote the set of all physical qualities (with this identification) of a quantum system $\mathcal{S}$ with corresponding Hilbert space $\mathbb{H}$ by $\mathfrak{P}(\mathbb{H})$. Notice, that a (nontrivial) member $\mathfrak{p}$ of $\mathfrak{P}(\mathbb{H})$ is a proposition specifying the value of some observable and is not an operator on $\mathbb{H}$ in some way or other related to the proposition $\mathfrak{p}$. The effect operator $p$ associated with a physical quality $\mathfrak{p}$ will be denoted by $p=\mathfrak{Z}(\mathfrak{p})$, thus defining a map $\mathfrak{Z}: \mathfrak{P}(\mathbb{H}) \rightarrow \mathfrak{E}(\mathbb{H})$.

It is worthwhile to mention that in the operational approach to quantum mechanics states are defined as equivalence classes of preparing instruments of a system, where two preparing instruments are said to be equivalent if they cannot be distinguished by measuring binary alternatives (Kraus, 1983; Ludwig, 1983). Moreover, effects can also be defined as equivalence classes of measuring instruments performing yes-no measurements, where two measuring instruments are called equivalent if for every state both measuring instruments are triggered with equal frequencies. Since, however, every measuring process can be thought of as being composed of elementary yes-no measurements of physical qualities (at least in principle) (Beltrametti and Cassinelli, 1981; Jauch, 1968) and since physical qualities represented by the same effect operator cannot be distinguished from each other by performing yes-no measurements, it follows that in quantum mechanics every pair of physical qualities which are represented by the same effect operator must be considered equivalent: that is, in quantum mechanics one is not interested in single physical qualities but in equivalence classes of physical qualities. We will call this equivalence classes of physical qualities PHYSICAL PROPERTIES OF THE PHYSICAL SYSTEM. It is reasonable to assume that physical properties of a quantum mechanical system are in one-to-one correspondence with effect operators (Kraus, 1983). Therefore in generalized quantum mechanics the effect operators represent the beables of the theory in the mathematical formalism. In the standard formulation of quantum mechanics often only the projection operators are referred to as representing 'properties' of the quantum system (following a terminology introduced by von Neumann (1932)). Thus effect operators are often referred to as representing so-called 'unsharp properties.' However, in general effect operators must not be considered as unsharp or smeared counterparts of some underlying more sharply defined properties but as independent entities in their own right. Since physical properties represented by effect operators can in general neither be measured ideally without disturbing the system nor measured repeatedly, one may say, loosely speaking, that projection operators represent stable properties and that all other effect operators represent unstable properties.

Again the probability of some physical property $F$ is in the state $\varrho$ given by $\operatorname{tr}(F \varrho)$.

Summing up, throughout this paper we adopt the following conventions: by a physically meaningful proposition about a system we mean a statement (associated with some effect operator) specifying the value of a generalized observable; we also talk about physical qualities in this connection; a proposition about a system is thus a statement in the semantical language of physics asserting the truth (or realization) of some physical qualities. By a physical property of a system we mean an equivalence class of physical qualities which cannot be distinguished by measuring binary alternatives. Physical properties are in one-one correspondence with effect 
operators.

We now turn to the discussion of histories in standard nonrelativistic quantum mechanics, which, loosely speaking, are defined to be sequences of projection operators on $\mathbb{H}$. In standard nonrelativistic quantum mechanics a history could alternatively be defined as time sequence of physical qualities since (as already remarked above and as will become clearer below) different physical qualities corresponding to the same projection operator can only simultaneously be true. Thus we stick to the usual definition

Definition $1 A$ Homogeneous History is a map $h: \mathbb{R} \rightarrow \mathcal{P}(\mathbb{H}), t \mapsto h_{t}$. We call $t_{i}(h):=$ $\min \left(t \in \mathbb{R} \mid h_{t} \neq 1\right)$ the INITIAL and $t_{f}(h):=\max \left(t \in \mathbb{R} \mid h_{t} \neq 1\right)$ the FINAL TIME of $h$ respectively. Furthermore, the SUPPORT OF $h$ is given by $\mathfrak{s}(h):=\left\{t \in \mathbb{R} \mid h_{t} \neq 1\right\}$. If $\mathfrak{s}(h)$ is finite, countable or uncountable, then we say that $h$ is a FINITE, COUNTABLE or UNCOUNTABLE HISTORY respectively. The space of all homogeneous histories will be denoted by $\mathcal{H}(\mathbb{H})$, the space of all finite homogeneous histories by $\mathcal{H}_{\text {fin }}(\mathbb{H})$ and the space of all finite homogeneous histories with support $S$ by $\mathcal{H}_{S}(\mathbb{H})$.

In this work we focus attention on finite histories. In the following we will identify every homogeneous history $h$ with the string of its nontrivial projection operators, i.e., we write $h \simeq\left\{h_{t_{k}}\right\}_{t_{k} \in \mathfrak{s}(h)}$.

For every finite subset $S$ of $\mathbb{R}$ we can consider the Hilbert tensor product $\otimes_{t \in S} \mathbb{H}$ and the algebra $\mathcal{B}_{S}^{\otimes}(\mathbb{H})$ of bounded linear operators on $\otimes_{t \in S} \mathbb{H}$. It has been pointed out by Isham (1994) that for any fixed $S$ there is an injective (but not surjective) correspondence $\sigma_{S}$ between finite histories with support $S$ and elements of $\mathcal{B}_{S}^{\otimes}(\mathbb{H})$ given by

$$
\sigma_{S}: \mathcal{H}_{S}(\mathbb{H}) \rightarrow \mathcal{B}_{S}^{\otimes}(\mathbb{H}), h \simeq\left\{h_{t_{k}}\right\}_{t_{k} \in S} \mapsto \otimes_{t_{k} \in S} h_{t_{k}}
$$

The finite homogeneous histories with support $S$ can therefore be identified with projection operators on $\otimes_{t \in S} \mathbb{H}$. The set of all projection operators on $\otimes_{t \in S} \mathbb{H}$ will in the sequel be denoted by $\mathcal{P}_{S}^{\otimes}(\mathbb{H})$. However, not all projection operators in $\mathcal{P}_{S}^{\otimes}(\mathbb{H})$ have the form $\sigma_{S}(h)$ with $h \in$ $\mathcal{H}_{S}(\mathbb{H})$.

If a homogeneous history vanishes for some $t_{0} \in \mathbb{R}$, i.e., $h_{t_{0}}=0$, then we say that $h$ is a ZERO HISTORY. All zero histories are collectively denoted by 0 , slightly abusing the notation.

Definition 2 Let $h, k \in \mathcal{H}(\mathbb{H})$. We say that $k$ is COARSER THAN $h$ if $h_{t} \leq k_{t}$ for all $t \in \mathbb{R}$ and write $h \leq k$. If furthermore $h \neq k$, then we write $h<k$. The set $\mathcal{H}(\mathbb{H})$ equipped with the relation $\leq$ is a partially ordered set.

Definition 3 Two homogeneous histories $h$ and $k$ are said to be DISJOINT if there is some $t \in \mathbb{R}$ such that $h_{t} k_{t}=0$. 
The identification of finite homogeneous histories with support $S$ with projection operators on $\otimes_{t \in S} \mathbb{H}$ allows for the introduction of a much broader class of histories. To this end we recall the well-known fact that the set $\mathcal{P}(\mathbb{H})$ of projection operators on a Hilbert space $\mathbb{H}$ carries the structure of an orthocomplemented complete lattice provided for $p_{1}, p_{2} \in \mathcal{P}(\mathbb{H})$ one defines [a] $p_{1} \leq p_{2}$ if $p_{1}$ projects on a subspace of the range of $p_{2},(\leq$ defines a partial order on $\mathcal{P}(\mathbb{H}))$, [b] the join $p_{1} \vee p_{2}$ of $p_{1}$ and $p_{2}$ to be the projection operator which projects on the smallest closed subspace of $\mathbb{H}$ which contains the subspaces $p_{1} \mathbb{H}$ and $p_{2} \mathbb{H}$, [c] the meet $p_{1} \wedge p_{2}$ of $p_{1}$ and $p_{2}$ to be the projection operator which projects on the intersection of $p_{1} \mathbb{H}$ and $p_{2} \mathbb{H}$ and [d] the orthocomplementation $\neg p_{1}$ of $p_{1}$ to be the projection operator which projects on the complement of $p_{1} \mathbb{H}$ in $\mathbb{H}$ (Birkhoff and von Neumann, 1936).

Definition 4 Let $S$ be a finite subset of $\mathbb{R}$, then we call the space $\mathcal{K}_{S}(\mathbb{H}):=\mathcal{P}_{S}^{\otimes}(\mathbb{H})$ of projection operators on $\otimes_{t \in S} \mathbb{H}$ the SPACE OF FINITE INHOMOGENEOUS HISTORIES WITH SupPORT $S$. The space of all finite inhomogeneous histories with arbitrary support will be denoted by $\mathcal{K}_{\text {fin }}(\mathbb{H})$ or by $\mathcal{P}_{\text {fin }}^{\otimes}(\mathbb{H})$.

The lattice operations on $\mathcal{P}_{S}^{\otimes}(\mathbb{H})$ induce corresponding operations on the finite homogeneous histories in $\mathcal{H}_{\text {fin }}(\mathbb{H})$, which are explicitly described in the following remarks.

Remark 3 Let $h, k \in \mathcal{H}_{\text {fin }}(\mathbb{H})$ be two finite homogeneous histories, then the JOIN $h \vee k$ of $h$ and $k$ is defined to be the unique finite history with support $\mathfrak{s}(h) \cup \mathfrak{s}(k)$ which is represented in $\mathcal{P}_{\mathfrak{s}(h) \cup \mathfrak{s}(k)}^{\otimes}(\mathbb{H})$ by $\left(\otimes_{t_{i} \in \mathfrak{s}(h)} h_{t_{i}}\right) \vee\left(\otimes_{s_{j} \in \mathfrak{s}(k)} k_{s_{j}}\right)$. The history $h \vee k$ may be not homogeneous. In this case $h \vee k$ is an inhomogeneous history. The JOIN $\bigvee_{j} h_{j}$ of any finite sequence $\left\{h_{j}\right\}$ of pairwise disjoint homogeneous histories is analogously defined to be the unique finite history with support $\bigcup_{j} \mathfrak{s}\left(h_{j}\right)$ which is represented in $\mathcal{P}_{\cup_{j} \mathfrak{s}\left(h_{j}\right)}^{\otimes}(\mathbb{H})$ by $\bigvee_{j}\left(\otimes_{t_{i} \in \mathfrak{s}\left(h_{j}\right)} h_{t_{i}}\right)$.

Remark 4 Let $h, k \in \mathcal{H}_{\text {fin }}(\mathbb{H})$ be two finite homogeneous histories, then the MEET $h \wedge k$ of $h$ and $k$ satisfies that $(h \wedge k)_{t}:=h_{t} \wedge k_{t}$ is the projection operator on the intersection of the ranges of $h_{t}$ and $k_{t}$ for all $t \in \mathbb{R}$. The meet operation maps pairs of finite homogeneous histories to a finite homogeneous history.

Remark 5 Let $h$ be a finite homogeneous history with support $\mathfrak{s}(h)$, then $\neg$ h is the unique history with support $\mathfrak{s}(h)$ which in $\mathcal{P}_{\text {fin }}^{\otimes}(\mathbb{H})$ is represented by $1-\bigotimes_{t \in \mathfrak{s}(h)} h_{t}$. We call $\neg$ the NEGATION of $h$. The negation $\neg$ of a finite homogeneous history $h$ may be inhomogeneous. Obviously the negation satisfies $h \vee \neg h=1$ and $h \wedge \neg h=0$. It is clear that $\neg h$ is uniquely determined by this two conditions.

Lemma 1 Let $S$ be a finite subset of $\mathbb{R}$, then the set $\mathcal{K}_{S}(\mathbb{H})$ is an orthocomplemented complete lattice.

Remark 6 The join, meet and orthocomplementation operations on $\mathcal{K}_{S}(\mathbb{H})$ (where $S$ is a finite subset of $\mathbb{R}$ ) and on $\mathcal{P}_{\text {fin }}^{\otimes}(\mathbb{H})$ are denoted by the same symbols (slightly abusing the notation). 
In (Isham, 1994) it is indicated how to imbed $\mathcal{K}_{S}(\mathbb{H})$ into an infinite tensor product of operator algebras and how to furnish the latter with a Hilbert lattice structure.

Definition 5 Two (possibly inhomogeneous) finite histories $h$ and $k$ are said to be DISJOINT if $h \leq \neg k$, where $\leq$ is the partial order on $\mathcal{K}_{\mathfrak{s}(h) \cup \mathfrak{s}(k)}(\mathbb{H})$. We write $h \perp k$.

Lemma 2 Let $h$ and $k$ denote two disjoint finite histories, then $h \wedge k=0$.

Definition 6 A history $h \in \mathcal{H}_{S}(\mathbb{H})$ is called a SIMPLE HISTORY if $h_{t}$ is a projection operator on a one dimensional subspace of $\mathbb{H}$ for every $t \in S$.

Lemma 3 For every finite $S \subset \mathbb{R}$ the space $\mathcal{P}_{S}^{\otimes}(\mathbb{H})$ can be generated from $\mathcal{H}_{S}(\mathbb{H})$ by the application of a countably infinite number of $\vee, \wedge$ and $\neg$ operations.

This follows from the fact that, say, in the case of the tensor product $\mathbb{H}_{1} \otimes \mathbb{H}_{2}$ the set $\left\{e_{n_{1}} \otimes e_{n_{2}}\right\}$ forms an orthonormal basis for $\mathbb{H}_{1} \otimes \mathbb{H}_{2}$ if and only if $\left\{e_{n_{1}}\right\}$ and $\left\{e_{n_{2}}\right\}$ form orthonormal bases for $\mathbb{H}_{1}$ and $\mathbb{H}_{2}$, respectively. A moments thought shows even more, namely

Lemma 4 For every finite $S \subset \mathbb{R}$ the set $\mathcal{P}_{S}^{\otimes}(\mathbb{H})$ can be generated from the set of all simple histories in $\mathcal{H}_{S}(\mathbb{H})$ by the application of a countably infinite number of $\vee$ operations.

Remark 7 For every finite $S \subset \mathbb{R}$ the meet, join and orthocomplementation operations on $\mathcal{P}_{S}^{\otimes}(\mathbb{H})$ induce a meet, join and an orthocomplementation operation on $\mathcal{P}_{\text {fin }}^{\otimes}(\mathbb{H})$ respectively which will be denoted by the same symbols.

Definition 7 Let $\mathcal{A}$ denote a finite collection $\left\{h_{k}\right\}$ of histories in $\mathcal{K}_{\text {fin }}(\mathbb{H})$. Then $\mathcal{A}$ is said to be DISJOINT if each pair of histories in $\mathcal{A}$ is disjoint. $\mathcal{A}$ is said to be COMPLETE if $\bigvee_{k} h_{k}=1$.

Furthermore, to every finite homogeneous history $h \in \mathcal{H}_{\text {fin }}(\mathbb{H})$ we associate its CLASS OPERATOR WITH RESPECT TO THE FIDUCIAL TIME $t_{0}$

$$
\begin{aligned}
C_{t_{0}}(h) & :=U\left(t_{0}, t_{n}\right) h_{t_{n}} U\left(t_{n}, t_{n-1}\right) h_{t_{n-1}} \ldots U\left(t_{2}, t_{1}\right) h_{t_{1}} U\left(t_{1}, t_{0}\right) \\
& =U\left(t_{0}, t_{i}(h)\right) h_{t_{n}}\left(t_{n}\right) h_{t_{n-1}}\left(t_{n-1}\right) \ldots h_{t_{1}}\left(t_{1}\right) U\left(t_{i}(h), t_{0}\right),
\end{aligned}
$$

where we have defined the Heisenberg picture operators

$$
h_{t_{k}}\left(t_{k}\right):=U\left(t_{k}, t_{i}(h)\right)^{\dagger} h_{t_{k}} U\left(t_{k}, t_{i}(h)\right)
$$

with respect to the initial time $t_{i}(h)$ of $h$. The class operators are extended to finite inhomogeneous histories by requiring that $C_{t_{0}}$ is additive in the following sense

$$
\begin{aligned}
C_{t_{0}}(h \vee k) & :=C_{t_{0}}(h)+C_{t_{0}}(k) \text { whenever } h \perp k \\
C_{t_{0}}(\neg h) & :=1-C_{t_{0}}(h) .
\end{aligned}
$$


This definitions are compatible with the lattice theoretical identities $\neg(h \vee k)=(\neg h) \wedge(\neg k)$ and $\neg(h \wedge k)=(\neg h) \vee(\neg k)$. Notice, that Equation 1 is a consequence of Equation 3. In the language of Birkhoff (1967) $C_{t_{0}}$ is an operator-valued VALUATION on $\mathcal{P}_{S}^{\otimes}(\mathbb{H})$ for every finite $S \subset \mathbb{R}$. It follows from a lemma in (Birkhoff, 1967, Chapter X.1.) that Equations 3 and 4 are equivalent to

$$
C_{t_{0}}(h \vee k):=C_{t_{0}}(h)+C_{t_{0}}(k), \text { whenever } h \perp k .
$$

The Equations 3 and $₫$ are motivated by the identities valid for all disjoint $h, k \in \mathcal{P}_{\text {fin }}^{\otimes}(\mathbb{H})$ :

$$
\begin{aligned}
h \vee k & =h+k, \\
\neg h & =1-h .
\end{aligned}
$$

The analogue of Equation 5 for inhomogeneous histories which are not finitely generated is

$$
C_{t_{0}}\left(\bigvee_{i=1}^{\infty} h_{i}\right):=\sum_{i=1}^{\infty} C_{t_{0}}\left(h_{i}\right), \text { if } h_{j} \perp\left(\bigvee_{i=1}^{j-1} h_{i}\right) \text { for every } j
$$

the series converging ultraweakly. It is clear that the left hand sides of Equation 3 and of Equation 6 are well-defined. This can be easily seen from Lemma 1 and from the definition of the tensor product $\otimes_{t \in S} \mathbb{H}$. We mention that the fiducial time $t_{0}$ can be chosen completely arbitrary.

By virtue of Lemma 4 it is therefore enough to know the class operators of every finite simple homogeneous history.

Definition 8 Let the state of a quantum mechanical system at time $t_{0}$ be given by the density operator $\varrho\left(t_{0}\right)$. For every pair $h$ and $k$ of finite homogeneous histories we define the DECOHERENCE WEIGHT OF $h$ AND $k$ by

$$
d_{\varrho}(h, k):=\operatorname{tr}\left(C_{t_{0}}(h) \varrho\left(t_{0}\right) C_{t_{0}}(k)^{\dagger}\right) .
$$

The functional $d_{\varrho}: \mathcal{H}_{\text {fin }}(\mathbb{H}) \times \mathcal{H}_{\text {fin }}(\mathbb{H}) \rightarrow \mathbb{C},(h, k) \mapsto d_{\varrho}(h, k)$ will be called the DECOHERENCE FUNCTIONAL ASSOCIATED WITH THE STATE $\varrho$. The decoherence functional is in an obvious way extended to finite inhomogeneous histories using Equations 3 and 6.

Lemma 5 Let $h, h^{\prime}$ and $k$ denote finite histories. The decoherence functional $d_{\varrho}$ satisfies

- $d_{\varrho}(h, h) \in \mathbb{R}$ and $d_{\varrho}(h, h) \geq 0$.

- $d_{\varrho}(h, k)=d_{\varrho}(k, h)^{*}$.

- $d_{\varrho}(1,1)=1$.

- $d_{\varrho}\left(h \vee h^{\prime}, k\right)=d_{\varrho}(h, k)+d_{\varrho}\left(h^{\prime}, k\right)$, whenever $h \perp h^{\prime}$.

- $d_{\varrho}(0, h)=0$, for all $h$. 
Now fix $h$ and vary $\varrho$ in $p_{\varrho}(h):=d_{\varrho}(h, h)$. Then $p_{\varrho}(h)$ is a positive, linear, bounded functional on $\mathcal{T}(\mathbb{H})_{1}^{+}$. Therefore it follows from Gleason's theorem that there exists a unique effect $F(h) \in \mathfrak{E}(\mathbb{H})$ such that

$$
d_{\varrho}(h, h)=\operatorname{tr}[\varrho F(h)] .
$$

Obviously

$$
F(h)=C_{t_{0}}(h)^{\dagger} C_{t_{0}}(h) .
$$

We call $F(h)$ the effect associated with the finite history $h$. The map $F: \mathcal{H}_{\text {fin }}(\mathbb{H}) \rightarrow \mathfrak{E}(\mathbb{H})$ is many-to-one and therefore information about $h$ is lost when considering $F(h)$ instead of $h$.

\section{Consistent sets of histories}

Definition 9 Let $h$ and $k$ be two disjoint histories in $\mathcal{P}_{\text {fin }}^{\otimes}(\mathbb{H})$. The histories $h$ and $k$ are said to be PRECONSISTENT With RESPECt to THE STATe $\varrho$ if Re $d_{\varrho}(h, k)=0$. Any collection $\mathcal{C}$ of histories in $\mathcal{P}_{\text {fin }}^{\otimes}(\mathbb{H})$ is said to be PRECONSISTENT WITH RESPECT TO THE STATE $\varrho$ if every pair of disjoint histories in $\mathcal{C}$ is preconsistent with respect to the state $\varrho$. Any collection $\mathcal{C}^{\prime}$ of histories in $\mathcal{P}_{\text {fin }}^{\otimes}(\mathbb{H})$ is said to be CONSISTENT WITH RESPECT TO THE STATE $\varrho$ if $\mathcal{C}^{\prime}$ is a Boolean algebra (with respect to the meet, join and orthocomplementation in $\mathcal{P}_{\text {fin }}^{\otimes}(\mathbb{H})$ ), see Remark 7 and with unit $1_{\mathcal{C}^{\prime}}$ ) and if $\mathcal{C}^{\prime}$ is preconsistent with respect to the state $\varrho$.

Note that our above terminology differs somewhat from the terminology used by other authors. Further, some authors call a pair $h, k$ of histories weakly decoherent if it satisfies $\operatorname{Re} d_{\varrho}(h, k)=0$ and mediumly decoherent if it satisfies $d_{\varrho}(h, k)=0$. There can be found other related notions of decoherence and consistency in the literature, see, e.g., (Gell-Mann and Hartle, 1995; Finkelstein, 1993; Zeh, n.d.). The condition Re $d_{\varrho}(h, k)=0$ is interpreted in physical terms by saying that the events $h$ and $k$ have vanishing interference in the state $\varrho$.

The notion of consistency is important because it is the key to a probability interpretation of the numbers $d_{\varrho}(h, h)$ for some (pre-)consistent sets of histories.

Let us recall that usually a probability space is defined to be a triple $(\Omega, \mathcal{A}, p)$, where $\Omega$ is an arbitrary set, $\mathcal{A}$ is a Boolean $\sigma$-algebra of subsets of $\Omega$ and $p$ is a probability measure on $\mathcal{A}$. This can be generalized as follows

Definition 10 Let $\mathcal{L}$ be a partially ordered set and $\mathcal{B} \subset \mathcal{L}$ be a Boolean lattice. A nonnegative valuation $m: \mathcal{B} \rightarrow \mathbb{R}^{+}$on $\mathcal{B}$ which is additive

$$
m\left[\bigvee_{k=1}^{N} \alpha_{k}\right]=\sum_{k=1}^{N} m\left[\alpha_{k}\right], \text { if } \alpha_{k} \wedge\left(\bigvee_{i=1}^{k-1} \alpha_{i}\right)=0, \text { for every } k<N
$$

is called a FINITE MEASURE ON $\mathcal{B}$. If $\mathcal{B}$ is a Borel lattice, then $N$ may be taken to be $\infty$. In this case $m$ is $\sigma$-additive. If $\mathcal{B}$ is not a Borel lattice, then $N$ is always finite. If furthermore $m\left[1_{\mathcal{B}}\right]=1$, then $m$ is called a PROBABILITy MEASURE ON $\mathcal{B}$ and the triple $(\mathcal{L}, \mathcal{B}, m)$ is called $a$ PROBABILITY LATTICE.

A Borel lattice is a Boolean $\sigma$-lattice (Birkhoff, 1967). 
Theorem 1 Let $\mathcal{C} \subset \mathcal{P}_{\text {fin }}^{\otimes}(\mathbb{H})$ be a Boolean lattice. If $\mathcal{C}$ is preconsistent with respect to the state $\varrho$, then the triple $\left(\mathcal{P}_{\text {fin }}^{\otimes}(\mathbb{H}), \mathcal{C}, p_{\varrho}\right)$ is a probability lattice, where $p_{\varrho}$ is defined by

$$
p_{\varrho}: \mathcal{C} \rightarrow \mathbb{R}^{+}, p_{\varrho}(h):=\frac{d_{\varrho}(h, h)}{d_{\varrho}\left(1_{\mathcal{C}}, 1_{\mathcal{C}}\right)} .
$$

The proof is straightforward.

In the literature it is often tacitly assumed that the preconsistent set of histories under consideration forms (or generates) a Boolean lattice so that a probability interpretation of the diagonal values of the decoherence functional makes sense.

The probability defined by Equation 8 can for finite homogeneous histories be interpreted as conditional probability, namely as the probability of the sequence of the propositions $h_{t_{f}}=$ $h_{t_{k}}, \ldots, h_{t_{k-j}}$ given that the sequence of propositions $h_{t_{k-j-1}}, \ldots, h_{t_{0}}$ is realized.

Lemma 6 Let $\left(\mathcal{P}_{\text {fin }}^{\otimes}(\mathbb{H}), \mathcal{C}, p_{\varrho}\right)$ be a probability lattice, where $p_{\varrho}$ is defined by Equation 8 , then for all $h, k \in \mathcal{C}$

- $0 \leq p_{\varrho}(h) \leq 1$

- $p_{\varrho}(h \vee k)+p_{\varrho}(h \wedge k)=p_{\varrho}(h)+p_{\varrho}(k)$.

- $p_{\varrho}(h) \leq p_{\varrho}(k)$ if $h \leq k$.

Corollary 1 Let $\mathcal{C} \subset \mathcal{P}_{\text {fin }}^{\otimes}(\mathbb{H})$ be a Boolean lattice. Then $\mathcal{C}$ is a preconsistent set of histories w.r.t. the state $\varrho$ if and only if every pair $h, k$ of histories in $\mathcal{C}$ satisfies

$$
d_{\varrho}(h \vee k, h \vee k)+d_{\varrho}(h \wedge k, h \wedge k)=d_{\varrho}(h, h)+d_{\varrho}(k, k) .
$$

Remark 8 We notice that $d_{\varrho}$ induces also probability functionals on sets of histories which are not Boolean lattices. Let $\mathcal{C}$ be a preconsistent set of pairwise disjoint histories, then $m_{\varrho}$ : $\mathcal{C} \rightarrow \mathbb{R}^{+}, m_{\varrho}(h):=d_{\varrho}(h, h) /\left(\sum_{k \in \mathcal{C}} d_{\varrho}(k, k)\right)$ is an additive functional on $\mathcal{C}$ and $m_{\varrho}(h)$ can be interpreted as probability of $h \in \mathcal{C}$. However, since $\mathcal{C}$ generates a Boolean sublattice of $\mathcal{K}_{\text {fin }}(\mathbb{H})$ on which $d_{\varrho}$ induces a probability measure extending $m_{\varrho}$, it is enough to consider Boolean algebras of histories.

\section{The logical interpretation of quantum mechanics}

The logical interpretation of (nonrelativistic) quantum mechanics is an epistemological interpretation of quantum mechanics. This interpretation is mainly due to Roland Omnès. In this section we first briefly outline some basic assertions of the logical interpretation of quantum mechanics. The logical interpretation as discussed in this section and Section 2 differs in some 
minor details from that in Omnès' original work (Omnès, 1988a-c, 1989, 1990, 1992, 1994, 1995).

The logical interpretation is a realistic interpretation of quantum mechanics and thus the discussion of Section 2 applies here, see in particular the first part of Section 2. In the logical formulation the most general propositions about a quantum mechanical system which have a physical meaning are identified with finite (or at least countably infinite) history propositions. Other statements about a system which cannot be casted into the framework of history propositions are not considered to be meaningful and hence excluded from consideration. A "probability" is associated with every history proposition in a consistent set. The set of all probabilities for all history propositions specifies the state of the system. In the Hilbert space formulation of quantum mechanics the state of a system is characterized by a density operator and the probabilities are given by Equation 8. The set of all meaningful propositions about some system in terms of histories together with their corresponding probabilities (given by Equation 8) is considered to be the most general conceivable knowledge about a particular quantum mechanical system. The probabilities of histories are considered to be objective entities in their own right, i.e., numbers associated with history propositions describing single systems, and not only as quantities approximately equal to frequencies in a series of measurements. We adopt again Popper's propensity interpretation for this probabilities, compare Remark 2 .

In our terminology introduced above the history propositions represent the (temporal) beables (in the sense of propensities) and the probabilities are therefore thought to express the (temporal) tendencies in the behaviour of the system. The probability measure on a consistent Boolean algebra of history propositions induced by the decoherence functional according to Theorem 11 defines in this consistent Boolean algebra two logical relations, namely an implication and an equivalence relation between histories. A history proposition $h$ is said to IMPLY a history proposition $k$ if the conditional probability $p_{\varrho}(k \mid h) \equiv \frac{p_{\varrho}(h \wedge k)}{p_{\varrho}(h)}$ is well-defined and equal to one. Two history propositions $h$ and $k$ are said to be EQUIVALENT if $h$ implies $k$ and vice versa.

The universal rule of interpretation of quantum mechanics can now be formulated as

Rule 1 Propositions about quantum mechanical systems should solely be expressed in terms of history propositions. Every description of an isolated quantum mechanical system should be expressed in terms of finite history propositions belonging to a common consistent Boolean algebra of histories. Every reasoning relating several propositions should be expressed in terms of the logical relations induced by the probability measure from Theorem 1 in that Boolean algebra.

This logical rule has to be understood as a semantical rule which systematizes the language of quantum mechanics. It once and for all makes sure, whether a reasoning or an implication is allowed or not. This is exactly what one expects from a rule building the basis of a complete interpretation. The logical rule can also be applied to the hitherto highly problematic retrodictive reasoning in quantum mechanics.

The causal relationship between different histories is coded into a logical relationship. This has nothing to do with introducing 'a new empirical kind of metalogic,' as is sometimes claimed (Zeh, n.d.), but simply introduces a convenient way of speaking: the logical rule is neither 
regarded as a scientific law of human thought nor intended to modify the logical structure of our mathematical reasoning, but it is rather confined to the systems of quantum mechanical propositions to which probabilities may be ascribed by the theory.

It should be noticed that in the above formulation there is neither a preferred history nor a preferred Boolean algebra in the theory, which may be associated in some way with the 'actual facts' observable in the real world. Omnès does not claim to have solved the problem of actualization of facts or the objectification problem in the quantum measurement process. On the contrary, Omnès argues in (Omnès, 1992, 1994) that it is neither feasible nor necessary that quantum mechanics provides an explanation for the process of actualization of facts. Therefore the criticism in this direction raised by Zeh (n.d.) is unfounded.

A few remarks are in order here. Firstly, this author does neither claim nor presuppose that quantum mechanics is the ultimate universal theory in terms of which every natural phenomenon can eventually be described. This may or may not be the case. No conclusive decision of this question can be made at the present.

Note 5 Some authors explicitly or implicitly postulate the universality of quantum mechanics casted into the framework of consistent histories. According to this postulate the formalism of nonrelativistic quantum mechanics (casted into the framework of consistent histories) can without significant changes be applied to the whole universe and every (approximately) isolated part of it. A globally defined notion of 'time translation' is needed simply to formulate such a theory. However, in general curved spacetimes there is no such globally defined preferred notion of 'time translation.' Thus this postulate needs further justification before it can be accepted as a generally valid fundamental principle of physics. Intimately related to this question is the question whether the notion of the initial state of the universe is meaningful at all. All these nontrivial problems can presumably be decided only on the basis of a not yet existing quantum theory of gravity.

Accordingly, we formulate the above Rule 1 modestly only for quantum mechanical systems, i.e., systems which can indeed be described by quantum mechanics.

Secondly, in his work Omnès identifies the possible 'properties' of a system at a fixed instant of time with projection operators. As already discussed in Section 2 we do not use this terminology in the present work.

Thirdly, Omnès has a good deal to say about decoherence by the external environment, about recovering classical physics and 'common sense' from his approach and about the notion of truth in quantum mechanics and related questions. It is beyond the scope of the present work to discuss these issues and thus the reader is referred to (Omnès, 1990, 1991, 1992, 1994, 1995; Dowker and Kent, 1996; Zeh, n.d.). Concerning the notion of truth, we recall that in the present work we adopt essentially Griffiths' point of view (Griffiths, 1995).

Fourthly, it is sometimes claimed that in realistic and individual interpretations of quantum mechanics it is possible to say that complementary physical qualities of a quantum system have at every instant of time definite values (Popper, 1982). This claim can be shown to have 
paradoxical consequences and is indeed forbidden by Rule 1 .

\section{Generalization of the Consistent Histories Approach}

\subsection{Motivation}

One drawback of the consistent histories formalism in its standard formulation is that the possible physical qualities of a physical system at any particular instant of time are restricted to ordinary physical qualities represented by projection operators.

The introduction of histories is usually motivated by saying that everything that can meaningfully be said about a quantum mechanical system can be expressed in terms of time sequences of one-time propositions about the system. As discussed above, in the usual formulation of quantum mechanics one-time propositions are identified with one-time physical qualities, which are represented by projection operators, so that histories are thought of as being correctly represented by time sequences of projection operators. However, as a matter of fact general physical qualities in quantum mechanics have to be represented by effects. Projection operators represent only special physical qualities in quantum mechanics.

Omnès asserts that the results of measurement theory can be deduced from the consistent history approach. Similar assertions can also be found in (Griffiths, 1984). In particular, he argues that in a series of measurements of some proposition (represented by a projection operator or a history proposition) the empirical frequencies of the results are approximately given by the probabilities associated with that proposition. However, Omnès restricts his discussion to a very limited class of measurement situations, see Chapter 8 in (Omnès, 1994). Firstly, he considers only measurements of ordinary observables with discrete eigenvalue spectrum and secondly only unitary measurement interactions which transform eigenstates of the measured observable into eigenstates of the same eigenvalue. In the language of (Busch et al., 1991) Omnès considers only normal unitary von Neumann (pre-)measurements. However, the modern quantum theory of measurement covers much more general measurement situations, specifically measurements of generalized observables, see (Busch et al., 1991).

Note 6 It follows from a theorem by Ozawa (1984) that as a matter of principle the consistent history approach in its standard formulation is unable to cover measurements of continuous observables. The restriction of the discussion of measurement theory to ordinary discrete observables in (Omnès, 1994) is therefore not only a matter of convenience. Continuous observables can only be dealt with after introducing a discrete reading scale. That is, continuous observables have to be replaced by discrete coarse-grained observables. However, such a procedure does in general manifestly destroy invariance properties of the continuous variables.

The interaction between the measurement apparatus and the measuring object in real measurements usually takes place during a finite time interval and cannot be associated with a fixed point of time. Further, many measurements have only a finite precision ('unsharp measurements') and cannot be considered to be measurements of physical qualities represented by 
projection operators or to be sequences of measurements of physical qualities represented by history propositions. As discussed by Ludwig (1983), Davies (1976), and by Busch, Lahti and Mittelstaedt (1991) the physical quality measured in general measurement situations has to be represented by some effect operator. However, the above formulated universal logical Rule 1 forbids to make predictions for or even to talk about results from such unsharp measurements and also forbids to draw any conclusions from them. This state of affairs is clearly unsatisfactory. Thus we feel that Omnès' logical rule has to be extended to cover also more realistic measurement situations.

The question arises whether the logical interpretation can be generalized such that generalized physical qualities can be dealt with and such that the more general measurement situations can be described by the consistent history approach to quantum mechanics.

Since projection valued measures represent observables which can be measured ideally and repeatedly (at least in principle) and since effect valued measures represent observables which in general cannot be measured ideally and repeatedly, one might be tempted to reject this argument and to argue apologetically that in the consistent history approach a quantum system is described by (and only by) the set of its 'properties' (which are usually identified with projection operators) which are asserted to be the fundamental ingredients in the description of quantum systems. According to this line of thought other (unsharp) observables are not fundamental and need thus not to be taken into account. However, we have already argued against this attitude in Section 2 and will not repeat our arguments here. It has been pointed out by Busch et al. (1989) that unsharp observables are 'far from being mere imperfections' and that this point of view 'amounts to a severe restriction of the measurement theoretic possibilities of quantum mechanics. For instance one could not interpret the Stern-Gerlach experiment as a measurement of a spin observable at all.' Here of course a real Stern-Gerlach experiment is meant and not one of its idealized textbook versions. The reader is referred to the lucid monograph by Busch, Grabowski and Lahti (1995).

One may also argue that Naimark's theorem (as stated, e.g., in (Busch et al., 1995)) implies that only PV measures need to be taken into account since POV measures can be replaced by PV measures on a larger Hilbert space $\widetilde{\mathbb{H}}$. [Naimark's theorem states that every maximal symmetric operator on a Hilbert space $\mathbb{H}$ is the restriction (to $\mathbb{H}$ ) of some self-adjoint operator on a larger Hilbert space $\widetilde{\mathbb{H}} \supset \mathbb{H}$.] However, the Hilbert space $\widetilde{\mathbb{H}}$ has in general no direct physical interpretation, and even if it has, then it typically represents the environment of the considered system or a measuring apparatus. In any case the PV measures on the Hilbert space $\widetilde{\mathbb{H}}$ can in general not be interpreted as describing solely the system under consideration and the projection operators in the range of the PV measures cannot in general be thought of as representing properties of the system [e.g., when $\widetilde{\mathbb{H}}$ describes a measuring situation on $\mathbb{H}$, then typically the PV measures arising from POV measures on $\mathbb{H}$ represent pointer observables of the measuring apparatus].

Therefore POV measures have to be considered as the observables in the theory and the question of the status of generalized observables and of the corresponding generalized physical qualities in the consistent history approach cannot be avoided. 
In summary of the above discussion we conclude that a generalization of the standard consistent histories approach is needed for the following reasons: Firstly, the notion of observable in the standard consistent histories scheme is restricted to the class of ordinary observables and secondly, it is not possible to describe realistic measurements in the language provided by the standard consistent

histories theory. It is the target of this section to generalize the consistent histories approach appropriately and thereby get rid of these drawbacks. In the first part of this section we construct the generalization of the consistent histories theory and of the logical interpretation for nonrelativistic quantum mechanics. At the end of this section we study the consequences of our results for the structure of more general temporal history theories in the spirit of (Isham, 1994). In Section 5 below we will furthermore briefly argue that our generalized history approach incorporates in a natural way histories of 'quasi-projectors' and that we can dispose of using approximate consistency conditions.

\subsection{The Space of Effect Histories}

As we have discussed above the equivalence classes of physical qualities of a system are the objects in the theory which are interpreted to represent possible events of physical reality. Thus in order to make pre- and retrodiction one has to study effect histories, i.e., sequences of effects on $\mathbb{H}$.

Definition 11 A homogeneous effect history is a map $u: \mathbb{R} \rightarrow \mathfrak{E}(\mathbb{H}), t \mapsto u_{t}$. The SUPPORT OF $u$ is given by $\mathfrak{s}(u):=\left\{t \in \mathbb{R} \mid u_{t} \neq 1\right\}$. If $\mathfrak{s}(u)$ is finite, countable or uncountable, then we say that $u$ is a FINITE, COUNTABLE or UNCOUNTABLE EFFECT HISTORY respectively. The space of all homogeneous effect histories will be denoted by $\mathbb{E}(\mathbb{H})$, the space of all finite homogeneous effect histories by $\mathbb{E}_{\text {fin }}(\mathbb{H})$ and the space of all finite homogeneous effect histories with support $S$ by $\mathbb{E}_{S}(\mathbb{H})$. All homogeneous effect histories for which there exists at least one $t \in \mathbb{R}$ such that $u_{t}=0$ are collectively denoted by 0 , slightly abusing the notation.

Remark 9 Let $G_{r}$ be a finite set with $r$ elements and supplied with two binary operations (free meet and free join) which will be denoted by $\wedge_{f r}$ and $\vee_{f r}$ respectively. Finite POLYNOMIALS in $G_{r}$ can be built from elements of $G_{r}$ by at most finitely many applications of $\wedge_{f r}$ and $\vee_{f r}$. On the space of all finite polynomials in $G_{r}$ a congruence relation is defined by imposing the algebraic identities valid in every lattice, see (Birkhoff, 1967). The FREE LATTICE $\mathcal{L}\left(G_{r}\right)$ GENERATED BY $G_{r}$ is the quotient space of the space of all finite polynomials in $G_{r}$ by this congruence relation. Now let $G_{\aleph}$ be a set with $\aleph$ elements, $\aleph$ any cardinal number. The free lattice $\mathcal{L}\left(G_{\aleph}\right)$ generated by $G_{\aleph}$ can be constructed as follows: for any finite subset $T \subset G_{\aleph}$ one has constructed $\mathcal{L}(T)$. Let $T$ and $S$ be two finite subsets of $G_{\aleph}$ satisfying $T \subset S$, then the canonical embedding $\eta_{T S}: T \rightarrow S$ can be extended to a monomorphism of free lattices $\bar{\eta}_{T S}: \mathcal{L}(T) \rightarrow \mathcal{L}(S)$ (Birkhoff, 1967, Theorem VI.16). Define

$$
\widetilde{\mathcal{L}}:=\bigcup_{\substack{T \subset G_{\aleph} \\ T \text { finite }}} \mathcal{L}(T) .
$$


Two elements $g_{1} \in \mathcal{L}\left(T_{1}\right)$ and $g_{2} \in \mathcal{L}\left(T_{2}\right)$ ( $T_{1}$ and $T_{2}$ finite) are EQUIVALENT if there is a $T_{12} \subset G_{\aleph}$ such that $T_{1} \subset T_{12}$ and $T_{2} \subset T_{12}$ and $\bar{\eta}_{T_{1} T_{12}}\left(g_{1}\right)=\bar{\eta}_{T_{2} T_{12}}\left(g_{2}\right)$. The FREE LATTICE $\mathcal{L}\left(G_{\aleph}\right)$ GENERATED BY $G_{\aleph}$ is now the quotient space of $\widetilde{\mathcal{L}}$ modulo this equivalence relation. It is easy to show that the lattice operations on $\mathcal{L}(T)$ induce the structure of a lattice on $\mathcal{L}\left(G_{\aleph}\right)$. The lattice operations on $\mathcal{L}\left(G_{\aleph}\right)$ will be denoted by $\wedge_{\mathcal{L}}$ and $\vee_{\mathcal{L}}$. More information about free lattices and free Boolean algebras can be found, e.g., in the monograph by Birkhoff (1967).

It is not clear at all how to define the meet and the join of two effect histories. Therefore the notion of inhomogeneous effect history cannot be defined in a natural way compatible with the partial ordering on $\mathfrak{E}(\mathbb{H})$. But we can define

Definition 12 The free lattice generated by $\mathbb{E}_{\text {fin }}(\mathbb{H})$ will be denoted by $\mathcal{L}\left(\mathbb{E}_{\text {fin }}(\mathbb{H})\right)$. The meet and the join operations in $\mathcal{L}\left(\mathbb{E}_{\text {fin }}(\mathbb{H})\right)$ will be denoted by $\wedge_{\mathcal{L}}$ and $\vee_{\mathcal{L}}$ respectively.

Remark 10 We can define a partial order on $\mathbb{E}_{\text {fin }}(\mathbb{H})$. For $u_{1}, u_{2} \in \mathbb{E}_{\text {fin }}(\mathbb{H})$ we set $u_{1} \leq u_{2}$ if $\left(u_{1}\right)_{t} \leq\left(u_{2}\right)_{t}$ for all $t \in \mathbb{R}$ and say that $u_{2}$ is COARSER than $u_{1}$. This partial ordering induces partially defined meet and join operations (denoted by $\wedge$ and $\vee$ ) on $\mathbb{E}_{\text {fin }}(\mathbb{H})$. Every finite polynomial in $\mathcal{L}\left(\mathbb{E}_{\text {fin }}(\mathbb{H})\right)$ can be transformed in EQUIVALENT polynomials by inserting the identifications $u_{1} \vee_{\mathcal{L}} u_{2}=u_{1} \vee u_{2}$ and $u_{1} \wedge_{\mathcal{L}} u_{2}=u_{1} \wedge u_{2}$ whenever the right hand sides are well-defined in $\mathbb{E}_{\text {fin }}(\mathbb{H})$. This defines an equivalence relation $\sim_{\mathbb{E}}$ on $\mathcal{L}\left(\mathbb{E}_{\text {fin }}(\mathbb{H})\right)$. It is clear that the physically interesting objects are the $\sim_{\mathbb{E}}$-equivalence classes in the quotient space $\mathcal{L}\left(\mathbb{E}_{\text {fin }}(\mathbb{H})\right) / \sim_{\mathbb{E}}$. By construction $\sim_{\mathbb{E}}$ is a congruence relation on $\mathcal{L}\left(\mathbb{E}_{\text {fin }}(\mathbb{H})\right)$. (For the notion of congruence relation see, e.g., (Birkhoff, 1967; Skornjakov, 1977).) The $\sim_{\mathbb{E}}$-congruence class of $u \in \mathbb{E}_{\text {fin }}(\mathbb{H})$ will be denoted by $\zeta(u)$. For $u \in \mathfrak{A} \subset \mathcal{L}\left(\mathbb{E}_{\text {fin }}(\mathbb{H})\right)$ we write $\zeta^{\mathfrak{A}}(u):=\left\{u^{\prime} \in\right.$ $\left.\mathfrak{A} \mid u^{\prime} \sim_{\mathbb{E}} u\right\}$ and $\zeta(\mathfrak{A}):=\{\zeta(a) \mid a \in \mathfrak{A}\}$.

Definition 13 The quotient space $\mathcal{L}\left(\mathbb{E}_{\text {fin }}(\mathbb{H})\right) / \sim_{\mathbb{E}}$ is called THE SPACE OF FINITE INHOMOGENEOUS EFFECT HISTORIES and will be denoted by $\widehat{\mathbb{E}}_{\text {fin }}(\mathbb{H})$. A finite inhomogeneous effect history induces a map $\hat{u}: \mathbb{R} \rightarrow \mathcal{L}(\mathfrak{E}(\mathbb{H})) / \sim_{\mathbb{E}}, t \mapsto \zeta(u)_{t}$, but not vice versa. The SUPPORT OF $\hat{u}$ is given by $\mathfrak{s}(\hat{u}):=\left\{t \in \mathbb{R} \mid \hat{u}_{t} \neq 1\right\}$. The space $\widehat{\mathbb{E}}(\mathbb{H})$ of GENERAL InHomogeneOus EFFECT HISTORIES and the space $\widehat{\mathbb{E}}_{S}(\mathbb{H})$ of INHOMOGENEOUS EFFECT HISTORIES WITH FINITE SUPPORT $S$ are defined analogously.

For homogeneous finite effect histories $u$ we define the class operator by

$$
\begin{aligned}
C_{t_{0}}(u) & :=U\left(t_{0}, t_{n}\right) \sqrt{u_{t_{n}}} U\left(t_{n}, t_{n-1}\right) \sqrt{u_{t_{n-1}}} \ldots U\left(t_{2}, t_{1}\right) \sqrt{u_{t_{1}}} U\left(t_{1}, t_{0}\right) \\
& =U\left(t_{0}, t_{i}(u)\right) \sqrt{u_{t_{n}}}\left(t_{n}\right) \sqrt{u_{t_{n-1}}}\left(t_{n-1}\right) \ldots \sqrt{u_{t_{1}}}\left(t_{1}\right) U\left(t_{i}(u), t_{0}\right),
\end{aligned}
$$

where we have defined the Heisenberg picture operators

$$
\sqrt{u_{t_{k}}}\left(t_{k}\right):=U\left(t_{k}, t_{i}(u)\right)^{\dagger} \sqrt{u_{t_{k}}} U\left(t_{k}, t_{i}(u)\right)
$$

with respect to the initial time $t_{i}(u)$ of $u$. 
For every pair $u$ and $v$ of finite homogeneous effect histories we define the DECOHERENCE WEIGHT of $u$ and $v$ by

$$
d_{\varrho}(u, v):=\operatorname{tr}\left(C_{t_{0}}(u) \varrho\left(t_{0}\right) C_{t_{0}}(v)^{\dagger}\right) .
$$

The functional $d_{\varrho}: \mathbb{E}_{f i n}(\mathbb{H}) \times \mathbb{E}_{f i n}(\mathbb{H}) \rightarrow \mathbb{C},(u, v) \mapsto d_{\varrho}(u, v)$ will be called the DECOHERENCE FUNCTIONAL ASSOCIATED WITH THE STATE $\varrho$. There immediately arises a serious difficulty with this decoherence functional. At first sight it seems difficult (if not impossible) to construct a natural mathematical structure on the space of effect histories such that the decoherence functional is additive in both arguments. Without this structure a consistency condition generalizing Equation 9 cannot even be formulated and an interpretation of $d_{\varrho}(u, u)$ as probability seems to be impossible. (We will see below that the decoherence functional induces a probability functional on some sets of effect histories on which the decoherence functional is not additive.) These questions are investigated in the next subsection.

Note 7 It is straightforward to generalize the notion of linearly positive history introduced by Goldstein and Page (1995) and to introduce linearly positive effect histories and the like. However, since the physical significance of the Goldstein-Page condition remains somewhat elusive, we will not consider it in this work.

\subsection{Consistent Effect Histories and the Generalized Logical Rule of Interpretation}

Isham (1994) has studied the logico-algebraic structure of the standard consistent histories approach and has discussed generalizations of this structure as models for more general history theories which may have applications to quantum space-time theories. It is the aim of this subsection to discuss this line of thought from the point of view of our generalized histories involving effects and to use our results to generalize the logical interpretation to the present framework. We start with some important definitions

Definition $14 A$ DIFFERENCE POSET or D-POSET is a partially ordered set $D$ with greatest element 1 and with a partial binary operation $\ominus: D_{2} \rightarrow D$, where $D_{2} \subset D \times D$, such that

- $b \ominus a$ is defined if and only if $a \leq b$ for all $a, b \in D$,

- $b \ominus a \leq b$ for all $a \leq b$,

- $b \ominus(b \ominus a)=a$, for all $a \leq b$,

- $a \leq b \leq c \Rightarrow c \ominus b \leq c \ominus a$ and $(c \ominus a) \ominus(c \ominus b)=b \ominus a$.

Difference posets have been introduced by Kôpka and Chovanec (1994) and have been further studied in (Kôpka, 1992; Dvurečenskij and Pulmannová 1994a-c; Dvurečenskij 1995; Pulmannová, 1995).

Definition $15 A$ set $D$ with two special elements $0,1 \in D$ supplied with a partially defined associative and commutative operation $\oplus: D_{2}^{\prime} \rightarrow D$, where $D_{2}^{\prime} \subset D \times D$, is called an EFFECT ALGEBRA if 
- For every $a \in D$ there exists a unique $a^{\prime} \in D$ such that $a \oplus a^{\prime}$ is defined and $a \oplus a^{\prime}=1$,

- If $1 \oplus b$ is defined, then $b=0$, for all $b \in D$.

An effect algebra $D$ is called an ORTHOALGEBRA if furthermore

- If $b \oplus b$ is defined, then $b=0$, for all $b \in D$.

Effect algebras have been introduced by Foulis and Bennett (1994). Whenever $a \oplus b$ is well-defined for $a, b \in D$, then we write $a \perp b$. Let $(D, \ominus)$ be a D-poset. Define

$$
a \oplus b:=1 \ominus((1 \ominus a) \ominus b),
$$

whenever the right hand side is well-defined. Then $\oplus$ is a well-defined partial binary operation on $D$ and $(D, \oplus)$ is an effect algebra. Conversely, let $(D, \oplus)$ be an effect algebra. Define

$$
b \ominus a:=\left(a \oplus b^{\prime}\right)^{\prime},
$$

whenever the right hand side is well-defined. Then $\ominus$ is a well-defined partially binary operation on $D$. Further, define $a \leq b$ for $a, b \in D$ if there exists $c \in D$ such that $c \perp a$ and $a \oplus c=b$. Then $(D, \ominus)$ is a D-poset. Therefore the notions of D-poset and effect algebra are equivalent and we will use both terms synonymously in the following.

Definition 16 A finite subset $\left\{a_{1}, a_{2}, \ldots, a_{n}\right\}$ of a $D$-poset $(D, \oplus)$ is said to be $\oplus$-ORTHOGONAL if $\bigoplus_{i=1}^{n} a_{i}:=a_{1} \oplus a_{2} \oplus \cdots \oplus a_{n}$ can be defined recursively. In this case $\bigoplus_{i=1}^{n} a_{i}$ is called the $\bigoplus$-SUM of $\left\{a_{1}, a_{2}, \ldots, a_{n}\right\}$. In particular, for every $n \in \mathbb{N}$, we define an $:=n a:=\bigoplus_{i=1}^{n} a$, for all $a \in D$ for which the right hand side is well-defined. $A \oplus$-ORTHOGONAL subset $\left\{a_{1}, a_{2}, \ldots, a_{n}\right\}$ of a D-poset $D$ is said to be COMPLETE if $\oplus_{i=1}^{n} a_{i}=1$. The family of all $\oplus$-sums of subsets of $\left\{a_{1}, a_{2}, \ldots, a_{n}\right\}$ will be denoted by $\oplus\left\{a_{1}, a_{2}, \ldots, a_{n}\right\}$.

Furthermore, let $D_{0} \subset D$, then the set of all well-defined finite $\oplus$-sums of elements of $D_{0}$ will be denoted by $\oplus D_{0}$.

Since $\oplus$ is commutative and associative, $\bigoplus_{i=1}^{n} a_{i}$ is indeed well-defined.

Remark 11 If $\left\{a_{1}, a_{2}, \ldots, a_{n}\right\}$ is a finite, complete, $\oplus$-orthogonal subset of a D-poset $(D, \oplus)$, then $\left(\oplus\left\{a_{1}, a_{2}, \ldots, a_{n}\right\}, \oplus\right)$ is itself a D-poset.

Remark 12 The set $\mathfrak{E}(\mathbb{H})$ of all effect operators on a Hilbert space $\mathbb{H}$ [with the scalar product denoted by $(\cdot, \cdot)]$ can be organized into a D-poset by defining a partial ordering and a partial binary operation $\ominus$ on $D$ by $A \leq B$ if $(A x, x) \leq(B x, x)$ for all $x \in \mathbb{H}$ and $C=B \ominus A$ if $(B x, x)-(A x, x)=(C x, x)$ for all $x \in \mathbb{H}$.

Definition 17 Let $(D, \oplus)$ be a D-poset. A Probability MEasure on $D$ is a map $p: D \rightarrow \mathbb{R}^{+}$ satisfying $p(1)=1$ and $p(a \oplus b)=p(a)+p(b)$, whenever $a \oplus b$ is well-defined. 
We next summarize the general axioms for a generalized quantum theory based on histories as stated by Isham (1994). According to Isham the algebraic structure underlying the generalized quantum mechanics of histories consists of the following ingredients:

1. The space of history propositions $\mathfrak{U}$.

- Isham and Linden (1994) suggest that the minimal useful mathematical structure on $\mathfrak{U}$ is that of an orthoalgebra. One may also consider the case that $\mathfrak{U}$ is an orthocomplemented lattice or has an even stronger structure (e.g., a complete orthocomplemented lattice). (Pulmannová (1995) proposed that the space of all histories in a generalized quantum theory may admit the structure of a D-poset. In the present work a physical justification of this proposal is given.)

2. The space of decoherence functionals.

A decoherence functional is a map $d: \mathfrak{U} \times \mathfrak{U} \rightarrow \mathbb{C}$ which satisfies for all $\alpha, \alpha^{\prime}, \beta \in \mathfrak{U}$

- $d(\alpha, \alpha) \in \mathbb{R}$ and $d(\alpha, \alpha) \geq 0$.

- $d(\alpha, \beta)=d(\beta, \alpha)^{*}$.

- $d(1,1)=1$.

- $d(0, \alpha)=0$, for all $\alpha$.

- $\quad$ - If $\mathfrak{U}$ is an orthoalgebra (or more generally an effect algebra), then $d\left(\alpha_{1} \oplus \alpha_{2}, \beta\right)=$ $d\left(\alpha_{1}, \beta\right)+d\left(\alpha_{2}, \beta\right)$ for all $\alpha_{1}, \alpha_{2}, \beta \in \mathfrak{U}$ with $\alpha_{1} \perp \alpha_{2}$.

- If $\mathfrak{U}$ is a lattice, then $d\left(\alpha_{1} \vee \alpha_{2}, \beta\right)=d\left(\alpha_{1}, \beta\right)+d\left(\alpha_{2}, \beta\right)-d\left(\alpha_{1} \wedge \alpha_{2}, \beta\right)$ for all $\alpha_{1}, \alpha_{2}, \beta \in \mathfrak{U}$.

3. The physical interpretation of the above axioms is the same as in the case of the standard consistent history formulation. One can define (pre)consistent sets of histories and interpret $d(\alpha, \alpha)$ as the probability of the history $\alpha$.

Besides the structures of the space of histories and the space of decoherence functionals which are believed to be present also in more general history theories than nonrelativistic quantum mechanics, Isham (1994) also describes structures arising in the history formulation of nonrelativistic quantum mechanics which may be an artefact of that theory and may be meaningless in more general quantum history theories.

4. The space $\mathcal{U}$ of history filters or homogeneous histories.

- $\mathcal{U}$ is a partial semigroup with composition law $\circ$. It satisfies $\alpha \circ 1=\alpha$ and $\alpha \circ 0=0$ for all $\alpha \in \mathcal{U}$. If $\alpha \circ \beta$ is defined, then $\alpha \circ \beta=\alpha \wedge \beta$.

- $\mathcal{U}$ is a partially ordered set with a unit 1 and a null history 0 . The partial order is denoted by $\leq$.

- On $\mathcal{U}$ an operation of meet, denoted by $\wedge$, is defined which satisfies $1 \wedge \alpha=\alpha$ and $0 \wedge \alpha=0$ for all $\alpha \in \mathcal{U}$. 
- There exists an embedding $\tau: \mathcal{U} \rightarrow \mathfrak{U}$, i.e., $\tau(U) \subset \mathfrak{U}$.

- $\mathfrak{U}$ can be generated from $\mathcal{U}$ by the application of a finite or countably infinite number of the algebraic operations defined on $\mathfrak{U}$.

Clearly, the axioms for $\mathcal{U}$ have to be modified when taking into account homogeneous effect histories. We will return to this point below. The definition of a semigroup will also be given below.

5. The space of temporal supports $\mathcal{S}$.

- $\mathcal{S}$ is a partial semigroup with unit.

- There exists a semigroup homomorphism $\kappa: \mathcal{U} \rightarrow \mathcal{S}$ such that $\kappa(1)=\kappa(0)=1 \in \mathcal{S}$.

$\mathcal{S}$ may contain only one element $\mathcal{S}=\{1\}$.

In the standard history approach to quantum mechanics we have the following identifications: $\mathcal{S}$ is the space of all finite subsets of $\mathbb{R}$ and $\mathcal{U}:=\mathcal{H}_{\text {fin }}(\mathbb{H})$. Isham's axioms, as stated above, are not the most general structure possible. There is great freedom to add further axioms to the list or to remove some (note that we have already omitted some of Isham's axioms). As discussed in Section 2, finite homogeneous histories can be identified with projection operators on $\otimes_{t \in S} \mathbb{H}$ in a natural way. One aim of this subsection is to examine to what extent Isham's results can be extended to our generalized effect histories and to explore its consequences for more general history theories. To this end the concept of the tensor product of effect algebras is of utmost importance. The tensor product of a pair of D-posets is defined by a universal mapping property, cf. (Dvurečenskij, 1995).

Definition 18 Let $D_{1}, D_{2}, \ldots, D_{n}$ and $L$ denote $D$-posets.

- A mapping $\phi: D_{1} \rightarrow L$ is said to be a MORPHISM if (i) $\phi(1)=1$, (ii) $a \perp b$ implies $\phi(a) \perp \phi(b)$ and $\phi(a \oplus b)=\phi(a) \oplus \phi(b)$, for all $a, b \in D_{1}$.

- A mapping $\phi: D_{1} \rightarrow L$ is said to be a MONOMORPHISM if (i) $\phi$ is a morphism and (ii) $\phi(a) \perp \phi(b)$ implies $a \perp b$, for all $a, b \in D_{1}$.

- A mapping $\phi: D_{1} \rightarrow L$ is said to be an ISOMORPHISM if $\phi$ is a surjective monomorphism.

- A mapping $\theta: D_{1} \times D_{2} \rightarrow L$ is said to be a SEMI-BIMORPHISM if

- $a, b \in D_{1}$ with $a \perp b$ imply $\theta(a, c) \perp \theta(b, c)$ and $\theta(a \oplus b, c)=\theta(a, c) \oplus \theta(b, c)$ for all $c \in D_{2}$;

- $c, d \in D_{2}$ with $c \perp d$ imply $\theta(a, c) \perp \theta(a, d)$ and $\theta(a, c \oplus d)=\theta(a, c) \oplus \theta(a, d)$ for all $a \in D_{1}$

- A mapping $\theta: D_{1} \times D_{2} \rightarrow L$ is said to be a BIMORPHISM if it is a semi-bimorphism and $\theta(1,1)=1$. 
- A mapping $\theta: D_{1} \times D_{2} \times \cdots \times D_{n} \rightarrow L$ is said to be a MULTimorPhism if it is a semi-bimorphism in every pair of its arguments and if $\theta(1, \ldots, 1)=1$.

Definition 19 Let $D_{1}, \ldots, D_{n}$ denote a family of $n$ D-posets. We say that a pair $(\mathfrak{D}, \Theta)$ consisting of a D-poset $\mathfrak{D}$ and a multimorphism $\Theta: D_{1} \times \cdots \times D_{n} \rightarrow \mathfrak{D}$ is a TENSOR PRODUCT of the family $D_{1}, \ldots, D_{n}$ if the following conditions are satisfied

- If $L$ is a D-poset and $\theta_{L}: D_{1} \times \cdots \times D_{n} \rightarrow L$ a multimorphism, then there exists a morphism $\beta: \mathfrak{D} \rightarrow L$ such that $\theta_{L}=\beta \circ \Theta$;

- Every element of $\mathfrak{D}$ is a finite orthogonal sum of elements of the form $\Theta\left(a_{1}, \ldots, a_{n}\right)$ with $a_{i} \in D_{i}$, for all $i$.

We also write $\mathfrak{D}=\bigotimes_{i=1}^{n} D_{i}=D_{1} \otimes \cdots \otimes D_{n}$ and $\Theta=\otimes$.

Dvurečenskij (1995) has proven that two D-posets $D_{1}$ and $D_{2}$ admit a tensor product if and only if there is a difference poset $L$ for which there is a bimorphism $\theta_{L}: D_{1} \times D_{2} \rightarrow L$. The definition of the tensor product of D-posets and Dvurečenskij's theorem can straightforwardly be extended to any finite number of D-posets, see (Pulmannová, 1995).

Let in the sequel $\mathbb{N}$ denote the set of positive integers including 0 . We adopt the following definitions

Definition $A$ set $\Omega$ with an operation $\top: \Omega \times \Omega \rightarrow \Omega$ is called a SEMIGRoup if the associative law is satisfied.

Definition A triple $(\Omega, \top, \odot)$ is called a SEMIRING if

- $(\Omega, \top)$ is an abelian semigroup with neutral element,

- $(\Omega, \odot)$ is a semigroup with unit 1 ,

- the distributive laws are satisfied.

Example: Let $\alpha \in \mathbb{R}^{+} \backslash\{0\}$ and define $\mathbb{N}_{\alpha}:=\left\{n^{\alpha} \mid n \in \mathbb{N}\right\}$. A semigroup operation $+_{\alpha}$ : $\mathbb{N}_{\alpha} \times \mathbb{N}_{\alpha} \rightarrow \mathbb{N}_{\alpha}$ is defined by $u+{ }_{\alpha} v:=\left(u^{1 / \alpha}+v^{1 / \alpha}\right)^{\alpha}$. Further, define $\odot_{\alpha}: \mathbb{N}_{\alpha} \times \mathbb{N}_{\alpha} \rightarrow \mathbb{N}_{\alpha}$ by $u \odot_{\alpha} v:=\left(u^{1 / \alpha} \cdot v^{1 / \alpha}\right)^{\alpha}=u \cdot v$. Then the triple $\left(\mathbb{N}_{\alpha},+_{\alpha}, \odot_{\alpha}\right)$ is a semiring. For all $\alpha \in \mathbb{R}^{+} \backslash\{0\}$ there is a semiring isomorphism $\iota_{\alpha}: \mathbb{N}_{\alpha} \hookrightarrow \mathbb{N}, \iota_{\alpha}(u):=u^{1 / \alpha}$. We call $\left(\mathbb{N}_{\alpha},+_{\alpha}, \odot_{\alpha}\right)$ the semiring of $\alpha$-SCALED NATURAL NUMBERS.

Definition Let $(M,+)$ be an abelian semigroup and $(\Omega,+, \odot)$ be a semiring and $\cdot: \Omega \times M \rightarrow M$ be a 'scalar multiplication.' Then the system $(M,+; \Omega, \odot ; \cdot)$ is called a SEMIMODULE OVER THE $\operatorname{SEMIRING}(\Omega,+, \odot)$ if for all $\omega, \omega_{1}, \omega_{2} \in \Omega$ and $m, m_{1}, m_{2} \in M$

- $\omega \cdot\left(m_{1}+m_{2}\right)=\omega \cdot m_{1}+\omega m_{2}$, 
- $\left(\omega_{1}+\omega_{2}\right) \cdot m=\omega_{1} \cdot m+\omega_{2} \cdot m$,

- $\left(\omega_{1} \odot \omega_{2}\right) \cdot m=\omega_{1} \cdot\left(\omega_{2} \cdot m\right)$,

- $1 \cdot m=m$.

Consider now two complex Hilbert spaces $\mathbb{H}_{1}$ and $\mathbb{H}_{2}$ and the corresponding sets of effects $\mathfrak{E}\left(\mathbb{H}_{1}\right)$ and $\mathfrak{E}\left(\mathbb{H}_{2}\right)$. An immediate consequence of Dvurečenskij's theorem is that the tensor product of $\mathfrak{E}\left(\mathbb{H}_{1}\right)$ and $\mathfrak{E}\left(\mathbb{H}_{2}\right)$ exists. To gain insight into the structure of the space of effect histories it is necessary to explicitly construct the tensor product of $\mathfrak{E}\left(\mathbb{H}_{1}\right)$ and $\mathfrak{E}\left(\mathbb{H}_{2}\right)$. To this end we regard $\mathbb{H}_{1}$ as right semimodule over $\mathbb{N}$ and $\mathbb{H}_{2}$ as left semimodule over $\mathbb{N}$ and denote by $\mathbb{H}_{1} \otimes_{\mathbb{N}} \mathbb{H}_{2}$ the tensor product of the $\mathbb{N}$-semimodules $\mathbb{H}_{1}$ and $\mathbb{H}_{2}$ in the category of semimodules over $\mathbb{N}$, that is, $\mathbb{H}_{1} \otimes_{\mathbb{N}} \mathbb{H}_{2}$ is the free abelian semigroup generated by elements of the form $\psi \otimes_{\mathbb{N}} \varphi$ with $\psi \in \mathbb{H}_{1}$ and $\varphi \in \mathbb{H}_{2}$ subject to the relations

1. $\left(\psi_{1}+\psi_{2}\right) \otimes_{\mathbb{N}} \varphi=\left(\psi_{1} \otimes_{\mathbb{N}} \varphi\right)+\left(\psi_{2} \otimes_{\mathbb{N}} \varphi\right)$, for all $\psi_{1}, \psi_{2} \in \mathbb{H}_{1}$ and $\varphi \in \mathbb{H}_{2} ;$

2. $\psi \otimes_{\mathbb{N}}\left(\varphi_{1}+\varphi_{2}\right)=\left(\psi \otimes_{\mathbb{N}} \varphi_{1}\right)+\left(\psi \otimes_{\mathbb{N}} \varphi_{2}\right)$, for all $\psi \in \mathbb{H}_{1}$ and $\varphi_{1}, \varphi_{2} \in \mathbb{H}_{2} ;$

3. $n\left(\psi \otimes_{\mathbb{N}} \varphi\right) \equiv(\psi n) \otimes_{\mathbb{N}} \varphi=\psi \otimes_{\mathbb{N}}(n \varphi)$, for all $\psi \in \mathbb{H}_{1}, \varphi \in \mathbb{H}_{2}$ and $n \in \mathbb{N}$.

To every $E_{1} \in \mathfrak{E}\left(\mathbb{H}_{1}\right)$ and $E_{2} \in \mathfrak{E}\left(\mathbb{H}_{2}\right)$ one can define a map $\mathbb{H}_{1} \otimes_{\mathbb{N}} \mathbb{H}_{2} \rightarrow \mathbb{H}_{1} \otimes_{\mathbb{N}} \mathbb{H}_{2}$ by

$$
E_{1} \otimes_{\mathbb{N}} E_{2}\left(\psi \otimes_{\mathbb{N}} \varphi\right):=E_{1} \psi \otimes_{\mathbb{N}} E_{2} \varphi
$$

$\mathfrak{E}\left(\mathbb{H}_{1}\right)$ and $\mathfrak{E}\left(\mathbb{H}_{2}\right)$ can also be regarded as right and left $\mathbb{N}$-semimodules respectively. We can thus consider the tensor product $\mathfrak{E}\left(\mathbb{H}_{1}\right) \otimes_{\mathbb{N}} \mathfrak{E}\left(\mathbb{H}_{2}\right)$ of $\mathfrak{E}\left(\mathbb{H}_{1}\right)$ and $\mathfrak{E}\left(\mathbb{H}_{2}\right)$ in the category of $\mathbb{N}$ semimodules. $\mathfrak{E}\left(\mathbb{H}_{1}\right) \otimes_{\mathbb{N}} \mathfrak{E}\left(\mathbb{H}_{2}\right)$ is the free abelian semigroup generated by elements of the form $E_{1} \otimes_{\mathbb{N}} E_{2}$, where $E_{1} \in \mathfrak{E}\left(\mathbb{H}_{1}\right)$ and $E_{2} \in \mathfrak{E}\left(\mathbb{H}_{2}\right)$ subject to the relations

1. $n\left(E_{1} \otimes_{\mathbb{N}} E_{2}\right) \equiv\left(E_{1} n\right) \otimes_{\mathbb{N}} E_{2}=E_{1} \otimes_{\mathbb{N}}\left(n E_{2}\right)$, for all $E_{1} \in \mathfrak{E}\left(\mathbb{H}_{1}\right), E_{2} \in \mathfrak{E}\left(\mathbb{H}_{2}\right)$ and $n \in \mathbb{N}$;

2. $\left(E_{1} \otimes_{\mathbb{N}} E_{2}\right)+\left(E_{1} \otimes_{\mathbb{N}} \tilde{E}_{2}\right)=E_{1} \otimes_{\mathbb{N}}\left(E_{2}+\tilde{E}_{2}\right)$, for all $E_{1} \in \mathfrak{E}\left(\mathbb{H}_{1}\right)$ and $E_{2}, \tilde{E}_{2} \in \mathfrak{E}\left(\mathbb{H}_{2}\right)$;

3. $\left(E_{1} \otimes_{\mathbb{N}} E_{2}\right)+\left(\tilde{E}_{1} \otimes_{\mathbb{N}} E_{2}\right)=\left(E_{1}+\tilde{E}_{1}\right) \otimes_{\mathbb{N}} E_{2}$, for all $E_{1}, \tilde{E}_{1} \in \mathfrak{E}\left(\mathbb{H}_{1}\right)$ and $E_{2} \in \mathfrak{E}\left(\mathbb{H}_{2}\right)$.

The scalar products on $\mathbb{H}_{1}$ and $\mathbb{H}_{2}$ allow to define a scalar product on $\mathbb{H}_{1} \otimes_{\mathbb{N}} \mathbb{H}_{2}$ given by

$$
\begin{array}{ll}
\langle\Phi|A| \Psi\rangle:=\left\langle\phi\left|E_{1}\right| \psi\right\rangle\left\langle\omega\left|E_{2}\right| \varphi\right\rangle, & \text { if } \Phi=\phi \otimes_{\mathbb{N}} \omega, \Psi=\psi \otimes_{\mathbb{N}} \varphi \in \mathbb{H}_{1} \otimes_{\mathbb{N}} \mathbb{H}_{2} \\
& \text { and } A=E_{1} \otimes_{\mathbb{N}} E_{2} \in \mathfrak{E}\left(\mathbb{H}_{1}\right) \otimes_{\mathbb{N}} \mathfrak{E}\left(\mathbb{H}_{2}\right) .
\end{array}
$$

This 'scalar product' is extended by linearity to arbitrary $A \in \mathfrak{E}\left(\mathbb{H}_{1}\right) \otimes_{\mathbb{N}} \mathfrak{E}\left(\mathbb{H}_{2}\right)$ and $\Phi, \Psi \in$ $\mathbb{H}_{1} \otimes_{\mathbb{N}} \mathbb{H}_{2}$. A partial order on $\mathfrak{E}\left(\mathbb{H}_{1}\right) \otimes_{\mathbb{N}} \mathfrak{E}\left(\mathbb{H}_{2}\right)$ can now be defined. For $A, B \in \mathfrak{E}\left(\mathbb{H}_{1}\right) \otimes_{\mathbb{N}} \mathfrak{E}\left(\mathbb{H}_{2}\right)$ we set $A \leq B$ if $\langle\Psi|A| \Psi\rangle \leq\langle\Psi|B| \Psi\rangle$ for all $\Psi \in \mathbb{H}_{1} \otimes_{\mathbb{N}} \mathbb{H}_{2}$.

A finite family $\left\{E_{i} \otimes_{\mathbb{N}} F_{i}\right\}_{i}$ of elements of $\mathfrak{E}\left(\mathbb{H}_{1}\right) \otimes_{\mathbb{N}} \mathfrak{E}\left(\mathbb{H}_{2}\right)$ is a DECOMPOSITION of $1 \otimes_{\mathbb{N}} 1$ if $\sum_{i} E_{i} \otimes_{\mathbb{N}} F_{i}=1 \otimes_{\mathbb{N}} 1$ in $\mathfrak{E}\left(\mathbb{H}_{1}\right) \otimes_{\mathbb{N}} \mathfrak{E}\left(\mathbb{H}_{2}\right)$. 
In order to establish the effect algebra structure on $\mathfrak{E}\left(\mathbb{H}_{1}\right) \otimes_{\mathbb{N}} \mathfrak{E}\left(\mathbb{H}_{2}\right)$ we define a partial binary operation $\oplus_{\mathbb{N}}$ on $\mathfrak{E}\left(\mathbb{H}_{1}\right) \otimes_{\mathbb{N}} \mathfrak{E}\left(\mathbb{H}_{2}\right)$ by

$$
A \oplus_{\mathbb{N}} B:=A+B \text {, if } A+B \leq 1,
$$

for all $A, B \in \mathfrak{E}\left(\mathbb{H}_{1}\right) \otimes_{\mathbb{N}} \mathfrak{E}\left(\mathbb{H}_{2}\right)$. However, $\left(\mathfrak{E}\left(\mathbb{H}_{1}\right) \otimes_{\mathbb{N}} \mathfrak{E}\left(\mathbb{H}_{2}\right), \oplus_{\mathbb{N}}\right)$ is not yet the tensor product of $\mathfrak{E}\left(\mathbb{H}_{1}\right)$ and $\mathfrak{E}\left(\mathbb{H}_{2}\right)$. Indeed, $\left(\mathfrak{E}\left(\mathbb{H}_{1}\right) \otimes_{\mathbb{N}} \mathfrak{E}\left(\mathbb{H}_{2}\right), \oplus_{\mathbb{N}}\right)$ is not even a D-poset.

We next define another partial binary operation $\oplus_{\mathfrak{D}}$ on $\mathfrak{E}\left(\mathbb{H}_{1}\right) \otimes_{\mathbb{N}} \mathfrak{E}\left(\mathbb{H}_{2}\right)$. We say that a $\oplus_{\mathfrak{D}^{-}}$ sum of the form $\left(E_{1} \otimes_{\mathbb{N}} F_{1}\right) \oplus_{\mathfrak{D}}\left(E_{2} \otimes_{\mathbb{N}} F_{2}\right) \oplus_{\mathfrak{D}} \cdots \oplus_{\mathfrak{D}}\left(E_{n} \otimes_{\mathbb{N}} F_{n}\right)$ of finitely many elements $E_{i} \otimes_{\mathbb{N}} F_{i} \in \mathfrak{E}\left(\mathbb{H}_{1}\right) \otimes_{\mathbb{N}} \mathfrak{E}\left(\mathbb{H}_{2}\right)$ exists if there exists a decomposition $\bigoplus_{\mathbb{N}, i} e_{i} \otimes_{\mathbb{N}} f_{i}$ of $1 \otimes_{\mathbb{N}} 1$ in $\mathfrak{E}\left(\mathbb{H}_{1}\right) \otimes_{\mathbb{N}} \mathfrak{E}\left(\mathbb{H}_{2}\right)$ such that every term of the form $E_{i} \otimes_{\mathbb{N}} F_{i}$ occurs also in the decomposition $\bigoplus_{\mathbb{N}, i \in I} e_{i} \otimes_{\mathbb{N}} f_{i}$ of $1 \otimes_{\mathbb{N}} 1$, i.e., if there for every $i \in\{1, \ldots, n\}$ there exists a $j \in I$ such that $E_{i} \otimes_{\mathbb{N}} F_{i}=e_{j} \otimes_{\mathbb{N}} f_{j}$.

In this case we also adopt the following way of speaking: we say that the $\oplus_{\mathfrak{D}}$-sum $\left(E_{1} \otimes_{\mathbb{N}} F_{1}\right) \oplus_{\mathfrak{D}}$ $\left(E_{2} \otimes_{\mathbb{N}} F_{2}\right) \oplus_{\mathfrak{D}} \cdots \oplus_{\mathfrak{D}}\left(E_{n} \otimes_{\mathbb{N}} F_{n}\right)$ is a $\oplus_{\mathfrak{D}}$-PART OF $1 \otimes_{\mathbb{N}} 1$.

In this case we set

$\left(E_{1} \otimes_{\mathbb{N}} F_{1}\right) \oplus_{\mathfrak{D}}\left(E_{2} \otimes_{\mathbb{N}} F_{2}\right) \oplus_{\mathfrak{D}} \cdots \oplus_{\mathfrak{D}}\left(E_{n} \otimes_{\mathbb{N}} F_{n}\right):=\left(E_{1} \otimes_{\mathbb{N}} F_{1}\right) \oplus_{\mathbb{N}}\left(E_{2} \otimes_{\mathbb{N}} F_{2}\right) \oplus_{\mathbb{N}} \cdots \oplus_{\mathbb{N}}\left(E_{n} \otimes_{\mathbb{N}} F_{n}\right)$.

We further say that a $\oplus_{\mathfrak{D}}$-sum $\left(E_{1} \otimes_{\mathbb{N}} F_{1}\right) \oplus_{\mathfrak{D}}\left(E_{2} \otimes_{\mathbb{N}} F_{2}\right) \oplus_{\mathfrak{D}} \cdots \oplus_{\mathfrak{D}}\left(E_{n} \otimes_{\mathbb{N}} F_{n}\right)$ is a MINIMAL REPRESENTATION of its associated $\oplus_{\mathfrak{D}}$-part of $1 \otimes_{\mathbb{N}} 1$ if the number of terms in the sum cannot be reduced by applying the defining relations 1., 2. and 3. of $\mathfrak{E}\left(\mathbb{H}_{1}\right) \otimes_{\mathbb{N}} \mathfrak{E}\left(\mathbb{H}_{2}\right)$ (see above).

The $\oplus_{\mathfrak{D}}$-sum $G_{\alpha} \oplus_{\mathfrak{D}} G_{\beta}$ of two arbitrary elements $G_{\alpha}$ and $G_{\beta} \in \mathfrak{E}\left(\mathbb{H}_{1}\right) \otimes_{\mathbb{N}} \mathfrak{E}\left(\mathbb{H}_{2}\right)$ is defined if $G_{\alpha}$ possesses a well-defined decomposition

$$
G_{\alpha}=\left(E_{\alpha_{1}} \otimes_{\mathbb{N}} F_{\alpha_{1}}\right) \oplus_{\mathfrak{D}} \cdots \oplus_{\mathfrak{D}}\left(E_{\alpha_{n}} \otimes_{\mathbb{N}} F_{\alpha_{n}}\right),
$$

and if $G_{\beta}$ possesses a well-defined decomposition

$$
G_{\beta}=\left(E_{\beta_{1}} \otimes_{\mathbb{N}} F_{\beta_{1}}\right) \oplus_{\mathfrak{D}} \cdots \oplus_{\mathfrak{D}}\left(E_{\beta_{m}} \otimes_{\mathbb{N}} F_{\beta_{m}}\right),
$$

(i.e., $G_{\alpha}$ and $G_{\beta}$ are both well-defined $\oplus_{\mathfrak{D}}$-parts of $1 \otimes_{\mathbb{N}} 1$ ) such that

$\left(G_{\alpha} \oplus_{\mathfrak{D}} G_{\beta}\right):=\left(E_{\alpha_{1}} \otimes_{\mathbb{N}} F_{\alpha_{1}}\right) \oplus_{\mathfrak{D}} \cdots \oplus_{\mathfrak{D}}\left(E_{\alpha_{n}} \otimes_{\mathbb{N}} F_{\alpha_{n}}\right) \oplus_{\mathfrak{D}}\left(E_{\beta_{1}} \otimes_{\mathbb{N}} F_{\beta_{1}}\right) \oplus_{\mathfrak{D}} \cdots \oplus_{\mathfrak{D}}\left(E_{\beta_{m}} \otimes_{\mathbb{N}} F_{\beta_{m}}\right)$

is well-defined. It is clear that $\left(G_{\alpha} \oplus_{\mathfrak{D}} G_{\beta}\right)$ such defined is independent of the particular decompositions considered. We say that two $\oplus_{\mathfrak{D}}$-parts of $1 \otimes_{\mathbb{N}} 1$, say, $G_{\delta}$ and $G_{\gamma}$ are EQUIVALENT if for all $\oplus_{\mathfrak{D}}$-parts $G$ of $1 \otimes_{\mathbb{N}} 1$ the following holds: $G \oplus_{\mathfrak{D}} G_{\delta}$ is well-defined and equal to $1 \otimes_{\mathbb{N}} 1$ if and only if $G \oplus_{\mathcal{D}} G_{\gamma}$ is well-defined and equal to $1 \otimes_{\mathbb{N}} 1$. We also write $G_{\delta} \sim_{\mathcal{D}} G_{\gamma}$. We write for the $\sim_{\mathfrak{D}}$-equivalence class of $G$ either $[G]$ or (abusing the notation) simply $G$. It is clear that the equivalence class of a homogeneous element $E \otimes_{\mathbb{N}} F$ contains only the element $E \otimes_{\mathbb{N}} F$, i.e., $\left[E \otimes_{\mathbb{N}} F\right]=\left\{E \otimes_{\mathbb{N}} F\right\}$. The partial addition $\oplus_{\mathfrak{D}}$ defined above for $\oplus_{\mathfrak{D}}$-parts of $1 \otimes_{\mathbb{N}} 1$ induces a partial addition on the equivalence classes of $\oplus_{\mathfrak{D}}$-parts of $1 \otimes_{\mathbb{N}} 1$ (denoted by the same symbol) by

$$
\left[G_{\delta}\right] \oplus_{\mathfrak{D}}\left[G_{\gamma}\right]:=\left[G_{\delta} \oplus_{\mathfrak{D}} G_{\gamma}\right],
$$


for all $G_{\delta}$ and $G_{\gamma}$ for which the right hand side is well-defined. It is clear that every equivalence class $[G]$ contains at most two elements. Further, if $[G]$ contains more than one element, then the complementary class $[G]^{\prime}:=\left[G^{\prime}\right]$ of $[G]$ (defined by $[G] \oplus_{\mathfrak{D}}[G]^{\prime}=[1]$ ) contains only one element but not vice versa. For well-defined $\oplus_{\mathfrak{D}}$-parts $G \in \mathfrak{E}\left(\mathbb{H}_{1}\right) \otimes_{\mathbb{N}} \mathfrak{E}\left(\mathbb{H}_{2}\right)$ of $1 \otimes_{\mathbb{N}} 1$ with a minimal representation of the form $G=\left(E_{1} \otimes_{\mathbb{N}} F_{1}\right) \oplus_{\mathfrak{D}}\left(E_{2} \otimes_{\mathbb{N}} F_{2}\right) \oplus_{\mathfrak{D}} \cdots \oplus_{\mathfrak{D}}\left(E_{n} \otimes_{\mathbb{N}} F_{n}\right)$ there are two candidates both of which may serve as complements in case they are well-defined, namely

$$
\begin{aligned}
\text { - } G^{*}:= & \left(E_{1} \otimes_{\mathbb{N}}\left(1-F_{1}\right)\right) \oplus_{\mathfrak{D}}\left(E_{2} \otimes_{\mathbb{N}}\left(1-F_{2}\right)\right) \oplus_{\mathfrak{D}} \cdots \oplus_{\mathfrak{D}}\left(E_{n} \otimes_{\mathbb{N}}\left(1-F_{n}\right)\right) \oplus_{\mathfrak{D}} \\
& \oplus_{\mathfrak{D}}\left(\left(1-\left(E_{1} \oplus E_{2} \oplus \cdots \oplus E_{n}\right)\right) \otimes_{\mathbb{N}} 1\right) \\
\text { - } G^{* *}:= & \left(\left(1-E_{1}\right) \otimes_{\mathbb{N}} F_{1}\right) \oplus_{\mathfrak{D}}\left(\left(1-E_{2}\right) \otimes_{\mathbb{N}} F_{2}\right) \oplus_{\mathfrak{D}} \cdots \oplus_{\mathfrak{D}}\left(\left(1-E_{n}\right) \otimes_{\mathbb{N}} F_{n}\right) \oplus_{\mathfrak{D}} \\
& \oplus_{\mathfrak{D}}\left(1 \otimes_{\mathbb{N}}\left(1-\left(F_{1} \oplus F_{2} \oplus \cdots \oplus F_{n}\right)\right)\right) .
\end{aligned}
$$

However, if $G$ is well-defined, it is clear that either $G^{*}$ or $G^{* *}$ is well-defined. (If they are both well-defined, then they are equivalent of course since also $G \oplus_{\mathfrak{D}} G^{*}$ and $G \oplus_{\mathfrak{D}} G^{* *}$ are both well-defined and equal to $1 \otimes_{\mathbb{N}}$ 1.) Define $\mathfrak{E}\left(\mathbb{H}_{1}\right) \widehat{\otimes}_{\mathfrak{D}} \mathfrak{E}\left(\mathbb{H}_{2}\right)$ as the effect algebra with partial binary operation $\oplus_{\mathfrak{D}}$ as the set consisting of all $\sim_{\mathfrak{D}}$-equivalence classes of well-defined finite $\oplus_{\mathfrak{D}}$-sums $G$ of elements of the form $E_{1} \widehat{\otimes}_{\mathfrak{D}} E_{2}:=E_{1} \otimes_{\mathbb{N}} E_{2} \in \mathfrak{E}\left(\mathbb{H}_{1}\right) \otimes_{\mathbb{N}} \mathfrak{E}\left(\mathbb{H}_{2}\right)$, i.e., consisting of all $\sim_{\mathfrak{D}}$-equivalence classes of $\oplus_{\mathfrak{D}}$-parts of $1 \otimes_{\mathbb{N}} 1$, subject to the relations

1. $n\left(E_{1} \widehat{\otimes}_{\mathfrak{D}} E_{2}\right)=\left(n E_{1}\right) \widehat{\otimes}_{\mathfrak{D}} E_{2}=E_{1} \widehat{\otimes}_{\mathfrak{D}}\left(n E_{2}\right)$, for all $E_{1} \in \mathfrak{E}\left(\mathbb{H}_{1}\right), E_{2} \in \mathfrak{E}\left(\mathbb{H}_{2}\right)$ and $n \in \mathbb{N}$, whenever the expressions are well-defined;

2. $\left(E_{1} \widehat{\otimes}_{\mathfrak{D}} E_{2}\right) \oplus_{\mathfrak{D}}\left(E_{1} \widehat{\otimes}_{\mathfrak{D}} \tilde{E}_{2}\right)=E_{1} \widehat{\otimes}_{\mathfrak{D}}\left(E_{2} \oplus \tilde{E}_{2}\right)$, for all $E_{1} \in \mathfrak{E}\left(\mathbb{H}_{1}\right)$ and $E_{2}, \tilde{E}_{2} \in \mathfrak{E}\left(\mathbb{H}_{2}\right)$, whenever the expressions are well-defined;

3. $\left(E_{1} \widehat{\otimes}_{\mathfrak{D}} E_{2}\right) \oplus_{\mathfrak{D}}\left(\tilde{E}_{1} \widehat{\otimes}_{\mathfrak{D}} E_{2}\right)=\left(E_{1} \oplus \tilde{E}_{1}\right) \widehat{\otimes}_{\mathfrak{D}} E_{2}$, for all $E_{1}, \tilde{E}_{1} \in \mathfrak{E}\left(\mathbb{H}_{1}\right)$ and $E_{2} \in \mathfrak{E}\left(\mathbb{H}_{2}\right)$, whenever the expressions are well-defined.

Theorem 2 The pair $\left(\mathfrak{E}\left(\mathbb{H}_{1}\right) \widehat{\otimes}_{\mathfrak{D}} \mathfrak{E}\left(\mathbb{H}_{2}\right), \oplus_{\mathfrak{D}}\right)$ is the tensor product of $\mathfrak{E}\left(\mathbb{H}_{1}\right)$ and $\mathfrak{E}\left(\mathbb{H}_{2}\right)$.

Proof: That every element $G$ of $\mathfrak{E}\left(\mathbb{H}_{1}\right) \widehat{\otimes}_{\mathfrak{D}} \mathfrak{E}\left(\mathbb{H}_{2}\right)$ is a finite $\oplus_{\mathfrak{D}}$-orthogonal sum of elements of the form $E_{1} \otimes_{\mathbb{N}} E_{2}$ with $E_{1} \in \mathfrak{E}\left(\mathbb{H}_{1}\right)$ and $E_{2} \in \mathfrak{E}\left(\mathbb{H}_{2}\right)$ and that to every $G \in \mathfrak{E}\left(\mathbb{H}_{1}\right) \widehat{\otimes}_{\mathfrak{D}} \mathfrak{E}\left(\mathbb{H}_{2}\right)$ there is a unique $G^{*} \in \mathfrak{E}\left(\mathbb{H}_{1}\right) \widehat{\otimes}_{\mathfrak{D}} \mathfrak{E}\left(\mathbb{H}_{2}\right)$ such that $G \oplus_{\mathfrak{D}} G^{*}=1 \otimes_{\mathbb{N}} 1 \in \mathfrak{E}\left(\mathbb{H}_{1}\right) \widehat{\otimes}_{\mathfrak{D}} \mathfrak{E}\left(\mathbb{H}_{2}\right)$ is clear by construction. Let $\left(\left(\mathfrak{D}, \widehat{\oplus}_{\mathfrak{D}}\right) ; \Theta\right)$ denote the tensor product of $\mathfrak{E}\left(\mathbb{H}_{1}\right)$ and $\mathfrak{E}\left(\mathbb{H}_{2}\right)$. Then there exists a morphism $\theta: \mathfrak{D} \rightarrow \mathfrak{E}\left(\mathbb{H}_{1}\right) \widehat{\otimes}_{\mathfrak{D}} \mathfrak{E}\left(\mathbb{H}_{2}\right)$ such that $\widehat{\otimes}_{\mathfrak{D}}=\theta \circ \Theta$. Every element in $\mathfrak{E}\left(\mathbb{H}_{1}\right) \widehat{\otimes}_{\mathfrak{D}} \mathfrak{E}\left(\mathbb{H}_{2}\right)$ is an equivalence class of $\oplus_{\mathfrak{D}}$-parts of decompositions of $1 \otimes_{\mathbb{N}} 1 \in \mathfrak{E}\left(\mathbb{H}_{1}\right) \hat{\otimes}_{\mathfrak{D}} \mathfrak{E}\left(\mathbb{H}_{2}\right)$. The map $\Theta \circ \widehat{\otimes}_{\mathfrak{D}}^{-1}: \widehat{\otimes}_{\mathfrak{D}}\left(\mathfrak{E}\left(\mathbb{H}_{1}\right) \times \mathfrak{E}\left(\mathbb{H}_{2}\right)\right) \rightarrow \mathfrak{D}$ maps the collection of all homogeneous terms (i.e., those of the form $e_{i} \otimes_{\mathbb{N}} f_{j}$ ) in every particular decomposition of $1 \otimes_{\mathbb{N}} 1$ to a complete, $\widehat{\oplus}_{\mathcal{D}}$-orthogonal subset of $\mathfrak{D}$. Thus $\Theta \circ \widehat{\otimes}_{\mathfrak{D}}^{-1}$ can be extended to a map which maps every equivalence class of $\oplus_{\mathfrak{D}}$-parts of $1 \otimes_{\mathbb{N}} 1$ to a $\widehat{\oplus}_{\mathfrak{D}}$-part of $1_{\mathfrak{D}}=\Theta \circ \widehat{\otimes}_{\mathfrak{D}}^{-1}\left(1 \otimes_{\mathbb{N}} 1\right)$. We denote this extension also by $\Theta \circ \widehat{\otimes}_{\mathfrak{D}}^{-1}$. Since then $\theta \circ \Theta \circ \widehat{\otimes}_{\mathfrak{D}}^{-1}=i d_{\mathfrak{E}\left(\mathbb{H}_{1}\right) \widehat{\otimes}_{\mathfrak{D}} \mathfrak{E}\left(\mathbb{H}_{2}\right)}$, it follows that $\theta$ is surjective. We show that $\theta$ is also injective. To this end consider $\mathfrak{d}_{1}, \mathfrak{d}_{2} \in \mathfrak{D}$, such that $\theta\left(\mathfrak{d}_{1}\right)=\theta\left(\mathfrak{d}_{2}\right) . \theta\left(\mathfrak{d}_{1}\right)$ and $\theta\left(\mathfrak{d}_{2}\right)$ are both 
equivalence classes of $\oplus_{\mathfrak{D}}$-parts of $\widehat{\otimes}_{\mathfrak{D}}(1,1)$. Thus $\Theta \circ \widehat{\otimes}_{\mathfrak{D}}^{-1}\left(\theta\left(\mathfrak{d}_{1}\right)\right)=\Theta \circ \widehat{\otimes}_{\mathfrak{D}}^{-1}\left(\theta\left(\mathfrak{d}_{2}\right)\right)$. Since $\mathfrak{D}$ is a D-poset, $\mathfrak{d}_{1}$ and $\mathfrak{d}_{2}$ are $\widehat{\oplus}_{\mathfrak{D}^{-}}$-sums of elements of the form $\mathfrak{d}_{1}^{i}=\Theta\left(e_{11}^{i}, e_{12}^{i}\right)$ and $\mathfrak{d}_{2}^{j}=\Theta\left(e_{21}^{j}, e_{22}^{j}\right)$, i.e., $\mathfrak{d}_{1}=\widehat{\oplus}_{\mathfrak{D}} \mathfrak{d}_{1}^{i}=\widehat{\oplus}_{\mathfrak{D}} \Theta\left(e_{11}^{i}, e_{12}^{i}\right)$ and $\mathfrak{d}_{2}=\widehat{\oplus}_{\mathfrak{D}} \mathfrak{d}_{2}^{j}=\widehat{\oplus}_{\mathfrak{D}} \Theta\left(e_{21}^{j}, e_{22}^{j}\right)$. With $\theta \circ \Theta=\widehat{\otimes}_{\mathfrak{D}}$ it follows $\Theta \circ \widehat{\otimes}_{\mathfrak{D}}^{-1}\left(\theta\left(\mathfrak{d}_{1}^{i}\right)\right)=\mathfrak{d}_{1}^{i}$ and thus $\Theta \circ \widehat{\otimes}_{\mathfrak{D}}^{-1}\left(\theta\left(\mathfrak{d}_{1}\right)\right)=\mathfrak{d}_{1}$ and similarly $\Theta \circ \widehat{\otimes}_{\mathfrak{D}}^{-1}\left(\theta\left(\mathfrak{d}_{2}\right)\right)=\mathfrak{d}_{2}$. Thus $\mathfrak{d}_{1}=\mathfrak{d}_{2}$. It follows from the above argument that $\theta^{-1}$ is also a morphism. Thus $\theta$ is an isomorphism between D-posets and therefore $\left(\mathfrak{D}, \widehat{\oplus}_{\mathfrak{D}}\right)=\left(\mathfrak{E}\left(\mathbb{H}_{1}\right) \widehat{\otimes}_{\mathfrak{D}} \mathfrak{E}\left(\mathbb{H}_{2}\right), \oplus_{\mathfrak{D}}\right)$.

This result can immediately be generalized to the tensor product of finitely many such effect algebras. With an obvious generalization of our above notation we have

Corollary 2 The pair $\left(\mathfrak{E}\left(\mathbb{H}_{1}\right) \widehat{\otimes}_{\mathfrak{D}} \mathfrak{E}\left(\mathbb{H}_{2}\right) \widehat{\otimes}_{\mathfrak{D}} \cdots \widehat{\otimes}_{\mathfrak{D}} \mathfrak{E}\left(\mathbb{H}_{n}\right), \oplus_{\mathfrak{D}}\right)$ is the tensor product of the family $\mathfrak{E}\left(\mathbb{H}_{1}\right), \ldots, \mathfrak{E}\left(\mathbb{H}_{n}\right)$.

In the sequel we denote by $\Theta$ the map defined in the proof of Theorem 2 given by

$$
\Theta: \mathfrak{E}\left(\mathbb{H}_{1}\right) \times \cdots \times \mathfrak{E}\left(\mathbb{H}_{n}\right) \rightarrow \mathfrak{E}\left(\mathbb{H}_{1}\right) \widehat{\otimes}_{\mathfrak{D}} \cdots \widehat{\otimes}_{\mathfrak{D}} \mathfrak{E}\left(\mathbb{H}_{n}\right), \Theta\left(u_{t_{1}}, \ldots, u_{t_{n}}\right):=u_{t_{1}} \widehat{\otimes}_{\mathfrak{D}} \cdots \widehat{\otimes}_{\mathfrak{D}} u_{t_{n}}
$$

Remember that the equivalence class $\Theta\left(u_{t_{1}}, \ldots, u_{t_{n}}\right)$ contains only the element $u_{t_{1}} \widehat{\otimes}_{\mathfrak{D}} \cdots \widehat{\otimes}_{\mathfrak{D}} u_{t_{n}}$. However, general equivalence classes $[G] \in \mathfrak{E}\left(\mathbb{H}_{1}\right) \widehat{\otimes}_{\mathfrak{D}} \cdots \widehat{\otimes}_{\mathfrak{D}} \mathfrak{E}\left(\mathbb{H}_{n}\right)$ contain at most $n$ ! elements.

Now we consider homogeneous effect histories with fixed finite support $S=\left\{t_{1}, \ldots, t_{n}\right\}$. The set of all such histories can be identified with the Cartesian product $\mathfrak{E}(\mathbb{H})_{t_{1}} \times \cdots \times \mathfrak{E}(\mathbb{H})_{t_{n}}$. The class operator defined by Equation 10 factorizes according to

$u=\left(u_{t_{1}}, \ldots, u_{t_{n}}\right) \stackrel{\Theta}{\longrightarrow} \Theta(u)=u_{t_{1}} \widehat{\otimes}_{\mathfrak{D}} \cdots \widehat{\otimes}_{\mathfrak{D}} u_{t_{n}} \stackrel{\sqrt{ }}{\longrightarrow} \sqrt{\Theta(u)}=u_{t_{1}}^{1 / 2} \widehat{\otimes}_{\mathfrak{D}} \cdots \widehat{\otimes}_{\mathfrak{D}} u_{t_{n}}^{1 / 2} \stackrel{C_{t_{0}}^{\prime}}{\longrightarrow} C_{t_{0}}^{\prime}(\sqrt{\Theta(u)})=C_{t_{0}}(u)$,

where $C_{t_{0}}^{\prime}$ is defined in an obvious way.

We define a decoherence functional $\widehat{d}_{\varrho, S}$ for pairs $(a, b)$ of homogeneous elements in $\left(\mathfrak{E}(\mathbb{H})_{t_{1}} \otimes_{\mathbb{N}} \cdots \otimes_{\mathbb{N}} \mathfrak{E}(\mathbb{H})_{t_{n}}\right) \times\left(\mathfrak{E}(\mathbb{H})_{t_{1}} \otimes_{\mathbb{N}} \cdots \otimes_{\mathbb{N}} \mathfrak{E}(\mathbb{H})_{t_{n}}\right)$ by

$$
\widehat{d}_{\varrho, S}(a, b):=\operatorname{tr}\left(C_{t_{0}}^{\prime}(\sqrt{a}) \varrho C_{t_{0}}^{\prime}(\sqrt{b})^{\dagger}\right) .
$$

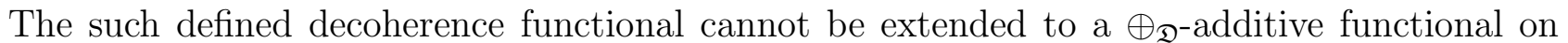
$\left(\mathfrak{E}(\mathbb{H})_{t_{1}} \widehat{\otimes}_{\mathfrak{D}} \cdots \widehat{\otimes}_{\mathfrak{D}} \mathfrak{E}(\mathbb{H})_{t_{n}}\right) \times\left(\mathfrak{E}(\mathbb{H})_{t_{1}} \widehat{\otimes}_{\mathfrak{D}} \cdots \widehat{\otimes}_{\mathfrak{D}} \mathfrak{E}(\mathbb{H})_{t_{n}}\right)$, that is, the D-poset structure given by $\oplus_{\mathfrak{D}}$ is not the physically interesting one.

The decoherence functional $\widehat{d}_{\varrho, S}$ can easily be extended to arbitrary finite homogeneous effect histories

$$
d_{\varrho, \mathfrak{N}}: \mathbb{E}_{f i n}(\mathbb{H}) \times \mathbb{E}_{f i n}(\mathbb{H}) \rightarrow \mathbb{C}, d_{\varrho, \mathfrak{N}}(u, v):=\widehat{d}_{\varrho, \mathfrak{s}(u) \cup \mathfrak{s}(v)}(\Theta(u), \Theta(v)),
$$

for all $u, v \in \mathbb{E}_{f i n}(\mathbb{H})$.

However, before we proceed to define and investigate the algebraic structure in terms of which the general consistency condition in our generalized history theory can be formulated, we briefly mention that there exist special sets of effect histories on which the decoherence functional induces a probability measure even though in general none of the familiar consistency 
conditions is satisfied for the elements of the special sets.

To this end fix an arbitrary finite homogeneous effect history $u_{0}$, fix a $t^{*}>t_{f}\left(u_{0}\right)$ and for every $E \in \mathfrak{E}(\mathbb{H})$ denote by $u_{E}$ the finite homogeneous effect history defined by $\mathfrak{s}\left(u_{E}\right):=\mathfrak{s}\left(u_{0}\right) \cup\left\{t^{*}\right\}$ and by

$$
\left(u_{E}\right)_{t}:=\left\{\begin{array}{rl}
\left(u_{0}\right)_{t} & : \quad t \neq t^{*} \\
E & : \quad t=t^{*}
\end{array},\right.
$$

that is, $u_{E}$ is the extension of $u_{0}$ by the effect $E$. The above decoherence functional $\widehat{d}_{\varrho, \mathfrak{s}\left(u_{0}\right) \cup\left\{t^{*}\right\}}$ induces a probability measure on the set $\widetilde{\mathfrak{E}}:=\left\{u_{E} \mid E \in \mathfrak{E}(\mathbb{H})\right\} \simeq \mathfrak{E}(\mathbb{H})$ by

$$
p_{\varrho}: \widetilde{\mathfrak{E}} \rightarrow \mathbb{R}, p_{\varrho}\left(u_{E}\right):=\widehat{d}_{\varrho, \mathfrak{s}\left(u_{0}\right) \cup\left\{t^{*}\right\}}\left(\Theta\left(u_{E}\right), \Theta\left(u_{E}\right)\right) .
$$

The D-poset structure on $\mathfrak{E}(\mathbb{H})$ induces a D-poset structure on $\widetilde{\mathfrak{E}}$. It is easy to see that $p_{\varrho}$ is additive on $\widetilde{\mathfrak{E}}$ in the sense $p_{\varrho}\left(u_{E_{1} \oplus E_{2}}\right)=p_{\varrho}\left(u_{E_{1}}\right)+p_{\varrho}\left(u_{E_{2}}\right)$, whenever $E_{1} \oplus E_{2}$ is well-defined. We will say that the D-poset of histories $\mathfrak{E}$ which can be constructed in this way (and which in particular is isomorphic to $\mathfrak{E}(\mathbb{H})$ ) is a FULL D-POSET OF EFFECT HISTORIES. If $E$ and $F$ are orthogonal projection operators, then $\widehat{d}_{\varrho, \mathfrak{s}\left(u_{0}\right) \cup\left\{t^{*}\right\}}\left(\Theta\left(u_{E}\right), \Theta\left(u_{F}\right)\right)=0$. However, if $E$ and $F$ are not orthogonal projection operators, then in general Re $\widehat{d}_{\varrho, \mathfrak{s}\left(u_{0}\right) \cup\left\{t^{*}\right\}}\left(\Theta\left(u_{E}\right), \Theta\left(u_{F}\right)\right) \neq 0$. The probability $p_{\varrho}\left(u_{E}\right)$ can be interpreted as the conditional probability that the event $E$ will be realized at time $t^{*}$ given that the history $u_{0}$ is realized. Stated differently, the probability of the history $u_{E}$ in the state $\varrho$ equals the probability of the effect $E$ in the (reduced) state $C_{t_{0}}\left(u_{0}\right) \varrho C_{t_{0}}\left(u_{0}\right)^{\dagger}$, i.e., $p_{\varrho}\left(u_{E}\right)=p_{C_{t_{0}}\left(u_{0}\right) \varrho C_{t_{0}}\left(u_{0}\right)^{\dagger}}(E)$.

We mention already here that it is possible to construct further exceptional D-posets of effect histories such that the decoherence functional is additive in both arguments. We will return to this point below.

We now return to our determination and investigation of the natural algebraic structure which makes the decoherence functional additive in both arguments.

It is possible to define a tensor product $\mathfrak{E}(\mathbb{H})_{t_{1}} \widetilde{\otimes}_{\mathfrak{D}} \cdots \widetilde{\otimes}_{\mathfrak{D}} \mathfrak{E}(\mathbb{H})_{t_{n}}$ such that the corresponding decoherence functional $d_{\varrho, S}$ is additive in both arguments as we will show next. To this end we again consider $\mathfrak{E}\left(\mathbb{H}_{1}\right) \otimes_{\mathbb{N}} \mathfrak{E}\left(\mathbb{H}_{2}\right)$ and define a partial binary operation $\widetilde{\oplus}_{\mathbb{N}}$ by

$$
A \widetilde{\oplus}_{\mathbb{N}} B:=\left(A^{1 / 2}+B^{1 / 2}\right)^{2}, \text { if } A^{1 / 2}+B^{1 / 2} \leq 1,
$$

for all homogeneous elements $A=A_{1} \otimes_{\mathbb{N}} A_{2}, B=B_{1} \otimes_{\mathbb{N}} B_{2} \in \mathfrak{E}\left(\mathbb{H}_{1}\right) \otimes_{\mathbb{N}} \mathfrak{E}\left(\mathbb{H}_{2}\right)$. Finite $\widetilde{\oplus}_{\mathbb{N}}$-sums of homogeneous elements are defined recursively and the set of all well-defined finite $\widetilde{\oplus}_{\mathbb{N}}$-sums of homogeneous elements will be denoted by $\mathfrak{E}\left(\mathbb{H}_{1}\right) \overline{\boldsymbol{\otimes}}_{\mathbb{N}} \mathfrak{E}\left(\mathbb{H}_{2}\right)$. Notice that $A \widetilde{\oplus}_{\mathbb{N}} B$ is a well-defined bounded, linear operator on $\mathbb{H}_{1} \otimes_{\mathbb{N}} \mathbb{H}_{2}$, but in general $A \widetilde{\oplus}_{\mathbb{N}} B \notin \mathfrak{E}\left(\mathbb{H}_{1}\right) \otimes_{\mathbb{N}} \mathfrak{E}\left(\mathbb{H}_{2}\right)$.

We have defined above a partial operation $\ominus$ on the space $\mathfrak{E}(\mathbb{H})$ of effect operators on the Hilbert space $\mathbb{H}$ such that $(\mathfrak{E}(\mathbb{H}), \ominus)$ is a D-poset. However, the D-poset structure on $\mathfrak{E}(\mathbb{H})$ is not unique. Define $A \widetilde{\oplus} B:=\left(A^{1 / 2}+B^{1 / 2}\right)^{2}$, for all $A, B \in \mathfrak{E}(\mathbb{H})$ satisfying $A^{1 / 2}+B^{1 / 2} \leq 1$. To prove that $(\mathfrak{E}(\mathbb{H}), \widetilde{\oplus})$ is a D-poset is straightforward. Moreover, $(\mathfrak{E}(\mathbb{H}), \widetilde{\oplus})$ is an $\mathbb{N}_{2}$-semimodule. In this work we denote the D-poset $(\mathfrak{E}(\mathbb{H}), \oplus)$ briefly by $\mathfrak{E}(\mathbb{H})$. When we refer to $\mathfrak{E}(\mathbb{H})$ supplied with the D-poset structure given by $\widetilde{\oplus}$, then we explicitly write $(\mathfrak{E}(\mathbb{H}), \widetilde{\oplus})$. 
We can now proceed as above and define a partial binary operation $\widetilde{\oplus}_{\mathfrak{D}}$ and construct the tensor product of $\left(\mathfrak{E}\left(\mathbb{H}_{1}\right), \widetilde{\oplus}\right)$ and $\left(\mathfrak{E}\left(\mathbb{H}_{2}\right), \widetilde{\oplus}\right)$ in the category of $\mathbb{N}_{2}$-semimodules. Everywhere in our above description of the definition of $\oplus_{\mathfrak{D}}$ preceding Theorem 2 we simply have to replace

- $\oplus$ by $\widetilde{\oplus}$;

- $\oplus_{\mathbb{N}}$ by $\widetilde{\oplus}_{\mathbb{N}}$;

- $\oplus_{\mathfrak{D}}$ by $\widetilde{\oplus}_{\mathfrak{D}}$;

- $\sim_{\mathfrak{D}}$ by $\approx_{\mathfrak{D}}$.

We say that a $\widetilde{\oplus}_{\mathfrak{D}}$-sum of the form $\left(E_{1} \otimes_{\mathbb{N}} F_{1}\right) \widetilde{\oplus}_{\mathfrak{D}}\left(E_{2} \otimes_{\mathbb{N}} F_{2}\right) \widetilde{\oplus}_{\mathfrak{D}} \cdots \widetilde{\oplus}_{\mathfrak{D}}\left(E_{n} \otimes_{\mathbb{N}} F_{n}\right)$ of finitely many elements $E_{i} \otimes_{\mathbb{N}} F_{i} \in \mathfrak{E}\left(\mathbb{H}_{1}\right) \otimes_{\mathbb{N}} \mathfrak{E}\left(\mathbb{H}_{2}\right)$ exists if there exists a decomposition $\widetilde{\bigoplus}_{\mathbb{N}, i} e_{i} \otimes_{\mathbb{N}} f_{i}$ of $1 \otimes_{\mathbb{N}} 1$ in $\mathfrak{E}\left(\mathbb{H}_{1}\right) \bar{\otimes}_{\mathbb{N}} \mathfrak{E}\left(\mathbb{H}_{2}\right)$ such that every term of the form $E_{i} \otimes_{\mathbb{N}} F_{i}$ occurs also in the decomposition $\widetilde{\bigoplus}_{\mathbb{N}, i \in I} e_{i} \otimes_{\mathbb{N}} f_{i}$ of $1 \otimes_{\mathbb{N}} 1$, i.e., if there for every $i \in\{1, \ldots, n\}$ there exists a $j \in I$ such that $E_{i} \otimes_{\mathbb{N}} F_{i}=e_{j} \otimes_{\mathbb{N}} f_{j}$.

In this case we also adopt the following way of speaking: we say that the $\widetilde{\oplus}_{\mathfrak{D}}$-sum $\left(E_{1} \otimes_{\mathbb{N}} F_{1}\right) \widetilde{\oplus}_{\mathfrak{D}}\left(E_{2} \otimes_{\mathbb{N}} F_{2}\right) \widetilde{\oplus}_{\mathfrak{D}}$. $\cdots \widetilde{\oplus}_{\mathfrak{D}}\left(E_{n} \otimes_{\mathbb{N}} F_{n}\right)$ is a $\widetilde{\oplus}_{\mathfrak{D}}$-PART OF $1 \otimes_{\mathbb{N}} 1$.

In this case we set

$$
\left(E_{1} \otimes_{\mathbb{N}} F_{1}\right) \widetilde{\oplus}_{\mathfrak{D}}\left(E_{2} \otimes_{\mathbb{N}} F_{2}\right) \widetilde{\oplus}_{\mathfrak{D}} \cdots \widetilde{\oplus}_{\mathfrak{D}}\left(E_{n} \otimes_{\mathbb{N}} F_{n}\right):=\left(E_{1} \otimes_{\mathbb{N}} F_{1}\right) \widetilde{\oplus}_{\mathbb{N}}\left(E_{2} \otimes_{\mathbb{N}} F_{2}\right) \widetilde{\oplus}_{\mathbb{N}} \cdots \widetilde{\oplus}_{\mathbb{N}}\left(E_{n} \otimes_{\mathbb{N}} F_{n}\right) .
$$

We further say that a $\widetilde{\oplus}_{\mathfrak{D}}$-sum $\left(E_{1} \otimes_{\mathbb{N}} F_{1}\right) \widetilde{\oplus}_{\mathfrak{D}}\left(E_{2} \otimes_{\mathbb{N}} F_{2}\right) \widetilde{\oplus}_{\mathfrak{D}} \cdots \widetilde{\oplus}_{\mathfrak{D}}\left(E_{n} \otimes_{\mathbb{N}} F_{n}\right)$ is a MinimaL REPRESENTATiON of its associated $\widetilde{\oplus}_{\mathfrak{D}}$-part of $1 \otimes_{\mathbb{N}} 1$ if the number of terms in the sum cannot be reduced by applying the following relations

1. $n\left(E_{1} \otimes_{\mathbb{N}} E_{2}\right) \equiv\left(n E_{1}\right) \otimes_{\mathbb{N}} E_{2}=E_{1} \otimes_{\mathbb{N}}\left(n E_{2}\right)$, for all $E_{1} \in\left(\mathfrak{E}\left(\mathbb{H}_{1}\right), \widetilde{\oplus}\right), E_{2} \in\left(\mathfrak{E}\left(\mathbb{H}_{2}\right), \widetilde{\oplus}\right)$ and $n \in \mathbb{N}_{2}$. whenever the expressions are well-defined;

2. $\left(E_{1} \otimes_{\mathbb{N}} E_{2}\right) \widetilde{\oplus}_{\mathfrak{D}}\left(E_{1} \otimes_{\mathbb{N}} \tilde{E}_{2}\right)=E_{1} \otimes_{\mathbb{N}}\left(E_{2} \widetilde{\oplus} \tilde{E}_{2}\right)$, for all $E_{1} \in\left(\mathfrak{E}\left(\mathbb{H}_{1}\right), \widetilde{\oplus}\right)$ and $E_{2}, \tilde{E}_{2} \in\left(\mathfrak{E}\left(\mathbb{H}_{2}\right), \widetilde{\oplus}\right)$, whenever the expressions are well-defined;

3. $\left(E_{1} \otimes_{\mathbb{N}} E_{2}\right) \widetilde{\oplus}_{\mathfrak{D}}\left(\tilde{E}_{1} \otimes_{\mathbb{N}} E_{2}\right)=\left(E_{1} \widetilde{\oplus} \tilde{E}_{1}\right) \otimes_{\mathbb{N}} E_{2}$, for all $E_{1}, \tilde{E}_{1} \in\left(\mathfrak{E}\left(\mathbb{H}_{1}\right), \widetilde{\oplus}\right)$ and $E_{2} \in\left(\mathfrak{E}\left(\mathbb{H}_{2}\right), \widetilde{\oplus}\right)$, whenever the expressions are well-defined.

The $\widetilde{\oplus}_{\mathfrak{D}}$-sum $\left(G_{\alpha} \widetilde{\oplus}_{\mathfrak{D}} G_{\beta}\right)$ of two arbitrary elements $G_{\alpha}$ and $G_{\beta} \in \mathfrak{E}\left(\mathbb{H}_{1}\right) \bar{\otimes}_{\mathbb{N}} \mathfrak{E}\left(\mathbb{H}_{2}\right)$ is defined if $G_{\alpha}$ possesses a well-defined decomposition

$$
G_{\alpha}=\left(E_{\alpha_{1}} \otimes_{\mathbb{N}} F_{\alpha_{1}}\right) \widetilde{\oplus}_{\mathfrak{D}} \cdots \widetilde{\oplus}_{\mathfrak{D}}\left(E_{\alpha_{n}} \otimes_{\mathbb{N}} F_{\alpha_{n}}\right),
$$

and if $G_{\beta}$ possesses a well-defined decomposition

$$
G_{\beta}=\left(E_{\beta_{1}} \otimes_{\mathbb{N}} F_{\beta_{1}}\right) \widetilde{\oplus}_{\mathfrak{D}} \cdots \widetilde{\oplus}_{\mathfrak{D}}\left(E_{\beta_{m}} \otimes_{\mathbb{N}} F_{\beta_{m}}\right),
$$

(i.e., $G_{\alpha}$ and $G_{\beta}$ are both well-defined $\widetilde{\oplus}_{\mathfrak{D}}$-parts of $1 \otimes_{\mathbb{N}} 1$ ) such that

$$
\left(G_{\alpha} \widetilde{\oplus}_{\mathfrak{D}} G_{\beta}\right):=\left(E_{\alpha_{1}} \otimes_{\mathbb{N}} F_{\alpha_{1}}\right) \widetilde{\oplus}_{\mathfrak{D}} \cdots \widetilde{\oplus}_{\mathfrak{D}}\left(E_{\alpha_{n}} \otimes_{\mathbb{N}} F_{\alpha_{n}}\right) \widetilde{\oplus}_{\mathfrak{D}}\left(E_{\beta_{1}} \otimes_{\mathbb{N}} F_{\beta_{1}}\right) \widetilde{\oplus}_{\mathfrak{D}} \cdots \widetilde{\oplus}_{\mathfrak{D}}\left(E_{\beta_{m}} \otimes_{\mathbb{N}} F_{\beta_{m}}\right)
$$

is well-defined. It is clear that $\left(G_{\alpha} \widetilde{\oplus}_{\mathfrak{D}} G_{\beta}\right)$ such defined is independent of the particular decompositions considered. We say that two $\widetilde{\oplus}_{\mathfrak{D}}$-parts of $1 \otimes_{\mathbb{N}} 1$, say, $G_{\delta}$ and $G_{\gamma}$ are EQUIVALENT if for all $\widetilde{\oplus}_{\mathfrak{D}}$-parts $G$ of $1 \otimes_{\mathbb{N}} 1$ the following holds: $G \widetilde{\oplus}_{\mathfrak{D}} G_{\delta}$ is well-defined and equal to $1 \otimes_{\mathbb{N}} 1$ if and only if $G \widetilde{\oplus}_{\mathfrak{D}} G_{\gamma}$ is well-defined and equal to $1 \otimes_{\mathbb{N}} 1$. We also write $G_{\delta} \approx_{\mathfrak{D}} G_{\gamma}$. We write for the $\approx_{\mathfrak{D}}$-equivalence class of $G$ either $[G]$ or (abusing the notation) simply $G$. It is clear that the equivalence class of a homogeneous element $E \otimes_{\mathbb{N}} F$ contains only the element $E \otimes_{\mathbb{N}} F$, i.e., $\left[E \otimes_{\mathbb{N}} F\right]=\left\{E \otimes_{\mathbb{N}} F\right\}$. The partial addition $\widetilde{\oplus}_{\mathfrak{D}}$ defined above for $\widetilde{\oplus}_{\mathfrak{D}}$-parts of $1 \otimes_{\mathbb{N}} 1$ induces a partial addition on the equivalence classes of $\widetilde{\oplus}_{\mathfrak{D}}$-parts of $1 \otimes_{\mathbb{N}} 1$ (denoted by the same symbol) by

$$
\left[G_{\delta}\right] \widetilde{\oplus}_{\mathfrak{D}}\left[G_{\gamma}\right]:=\left[G_{\delta} \widetilde{\oplus}_{\mathfrak{D}} G_{\gamma}\right]
$$


for all $G_{\delta}$ and $G_{\gamma}$ for which the right hand side is well-defined. It is clear that every equivalence class $[G]$ contains at most two elements. Further, if $[G]$ contains more than one element, then the complementary class $[G]^{\prime}:=\left[G^{\prime}\right]$ of $[G]$ (defined by $[G] \widetilde{\oplus}_{\mathfrak{D}}[G]^{\prime}=[1]$ ) contains only one element but not vice versa. For well-defined $\widetilde{\oplus}_{\mathcal{D}}$-parts $G \in \mathfrak{E}\left(\mathbb{H}_{1}\right) \otimes_{\mathbb{N}} \mathfrak{E}\left(\mathbb{H}_{2}\right)$ of $1 \otimes_{\mathbb{N}} 1$ with a minimal representation of the form $G:=\left(E_{1} \otimes_{\mathbb{N}} F_{1}\right) \widetilde{\oplus}_{\mathfrak{D}}\left(E_{2} \otimes_{\mathbb{N}} F_{2}\right) \widetilde{\oplus} \mathfrak{D} \cdots$ $\widetilde{\oplus}_{\mathfrak{D}}\left(E_{n} \otimes_{\mathbb{N}} F_{n}\right)$ there are two candidates both of which may serve as complements in case they are well-defined, namely

$$
\begin{aligned}
-G^{*}:= & \left(E_{1} \otimes_{\mathbb{N}}\left(1-F_{1}^{1 / 2}\right)^{2}\right) \widetilde{\oplus}_{\mathfrak{D}}\left(E_{2} \otimes_{\mathbb{N}}\left(1-F_{2}^{1 / 2}\right)^{2}\right) \widetilde{\oplus}_{\mathfrak{D}} \cdots \widetilde{\oplus}_{\mathfrak{D}}\left(E_{n} \otimes_{\mathbb{N}}\left(1-F_{n}^{1 / 2}\right)^{2}\right) \widetilde{\oplus}_{\mathfrak{D}} \\
& \widetilde{\oplus}_{\mathfrak{D}}\left(\left(1-\left(E_{1}^{1 / 2} \widetilde{\oplus}_{2}^{1 / 2} \widetilde{\oplus} \cdots \widetilde{\oplus} E_{n}^{1 / 2}\right)^{2}\right) \otimes_{\mathbb{N}} 1\right) \\
\bullet G^{* *}:= & \left(\left(1-E_{1}^{1 / 2}\right)^{2} \otimes_{\mathbb{N}} F_{1}\right) \widetilde{\oplus}_{\mathfrak{D}}\left(\left(1-E_{2}^{1 / 2}\right)^{2} \otimes_{\mathbb{N}} F_{2}\right) \widetilde{\oplus}_{\mathfrak{D}} \cdots \widetilde{\oplus}_{\mathfrak{D}}\left(\left(1-E_{n}^{1 / 2}\right)^{2} \otimes_{\mathbb{N}} F_{n}\right) \widetilde{\oplus}_{\mathfrak{D}} \\
& \widetilde{\oplus}_{\mathfrak{D}}\left(1 \otimes_{\mathbb{N}}\left(1-\left(F_{1}^{1 / 2} \widetilde{\oplus} F_{2}^{1 / 2} \widetilde{\oplus} \cdots \widetilde{\oplus} F_{n}^{1 / 2}\right)^{2}\right)\right) .
\end{aligned}
$$

However, if $G$ is well-defined, it is clear that either $G^{*}$ or $G^{* *}$ is also well-defined. (If they are both well-defined, then they are equivalent of course since then also $G \widetilde{\oplus}_{\mathfrak{D}} G^{*}$ and $G \widetilde{\oplus}_{\mathfrak{D}} G^{* *}$ are both well-defined and equal to $1 \otimes_{\mathbb{N}}$ 1.) Define $\mathfrak{E}\left(\mathbb{H}_{1}\right) \widetilde{\otimes}_{\mathfrak{D}} \mathfrak{E}\left(\mathbb{H}_{2}\right)$ as the effect algebra with partial binary operation $\widetilde{\oplus}_{\mathfrak{D}}$ as the set consisting of all $\approx_{\mathcal{D}}$-equivalence classes of well-defined finite $\widetilde{\oplus}_{\mathcal{D}}$-sums $G$ of elements of the form $E_{1} \widetilde{\otimes}_{\mathfrak{D}} E_{2}:=E_{1} \otimes_{\mathbb{N}} E_{2} \in$ $\mathfrak{E}\left(\mathbb{H}_{1}\right) \otimes_{\mathbb{N}} \mathfrak{E}\left(\mathbb{H}_{2}\right)$, i.e., consisting of all $\approx_{\mathfrak{D}}$-equivalence classes of $\widetilde{\oplus}_{\mathfrak{D}}$-parts of $1 \otimes_{\mathbb{N}} 1$, subject to the relations

1. $n\left(E_{1} \widetilde{\otimes}_{\mathfrak{D}} E_{2}\right) \equiv\left(n E_{1}\right) \widetilde{\otimes}_{\mathfrak{D}} E_{2}=E_{1} \widetilde{\otimes}_{\mathfrak{D}}\left(n E_{2}\right)$, for all $E_{1} \in\left(\mathfrak{E}\left(\mathbb{H}_{1}\right), \widetilde{\oplus}\right), E_{2} \in\left(\mathfrak{E}\left(\mathbb{H}_{2}\right), \widetilde{\oplus}\right)$ and $n \in \mathbb{N}_{2}$. whenever the expressions are well-defined;

2. $\left(E_{1} \widetilde{\otimes}_{\mathfrak{D}} E_{2}\right) \widetilde{\oplus}_{\mathfrak{D}}\left(E_{1} \widetilde{\otimes}_{\mathfrak{D}} \tilde{E}_{2}\right)=E_{1} \widetilde{\otimes}_{\mathfrak{D}}\left(E_{2} \widetilde{\oplus} \tilde{E}_{2}\right)$, for all $E_{1} \in\left(\mathfrak{E}\left(\mathbb{H}_{1}\right), \widetilde{\oplus}\right)$ and $E_{2}, \tilde{E}_{2} \in\left(\mathfrak{E}\left(\mathbb{H}_{2}\right), \widetilde{\oplus}\right)$, whenever the expressions are well-defined;

3. $\left(E_{1} \widetilde{\otimes}_{\mathfrak{D}} E_{2}\right) \widetilde{\oplus}_{\mathfrak{D}}\left(\tilde{E}_{1} \widetilde{\otimes}_{\mathfrak{D}} E_{2}\right)=\left(E_{1} \widetilde{\oplus} \tilde{E}_{1}\right) \widetilde{\otimes}_{\mathfrak{D}} E_{2}$, for all $E_{1}, \tilde{E}_{1} \in\left(\mathfrak{E}\left(\mathbb{H}_{1}\right), \widetilde{\oplus}\right)$ and $E_{2} \in\left(\mathfrak{E}\left(\mathbb{H}_{2}\right), \widetilde{\oplus}\right)$, whenever the expressions are well-defined.

Lemma 7 The pair $\left(\mathfrak{E}\left(\mathbb{H}_{1}\right) \widetilde{\otimes}_{\mathfrak{D}} \mathfrak{E}\left(\mathbb{H}_{2}\right), \widetilde{\oplus}_{\mathfrak{D}}\right)$ is the tensor product of the D-posets $\left(\mathfrak{E}\left(\mathbb{H}_{1}\right), \widetilde{\oplus}\right)$ and $\left(\mathfrak{E}\left(\mathbb{H}_{2}\right), \widetilde{\oplus}\right)$ in the category of $\mathbb{N}_{2}$-semimodules.

Lemma 0 can easily be extended to any finite family $\mathfrak{E}\left(\mathbb{H}_{1}\right), \ldots, \mathfrak{E}\left(\mathbb{H}_{n}\right)$. The decoherence functional $\widehat{d}_{\varrho, S}$ defined above in Equation 14 for pairs $(a, b)$ of homogeneous elements in $\left(\mathfrak{E}(\mathbb{H})_{t_{1}} \otimes_{\mathbb{N}} \cdots \otimes_{\mathbb{N}} \mathfrak{E}(\mathbb{H})_{t_{n}}\right) \times\left(\mathfrak{E}(\mathbb{H})_{t_{1}} \otimes_{\mathbb{N}} \cdots \otimes_{\mathbb{N}} \mathfrak{E}(\mathbb{H})_{t_{n}}\right)$ can now be extended to an additive decoherence functional $d_{\varrho, S}$ on $\left(\mathfrak{E}(\mathbb{H})_{t_{1}} \widetilde{\otimes}_{\mathfrak{D}} \cdots \widetilde{\otimes}_{\mathfrak{D}} \mathfrak{E}(\mathbb{H})_{t_{n}}\right) \times\left(\mathfrak{E}(\mathbb{H})_{t_{1}} \widetilde{\otimes}_{\mathfrak{D}} \cdots \widetilde{\otimes}_{\mathfrak{D}} \mathfrak{E}(\mathbb{H})_{t_{n}}\right)$.

Theorem 3 The decoherence functional $d_{\varrho, S}:\left(\mathfrak{E}(\mathbb{H})_{t_{1}} \widetilde{\otimes}_{\mathfrak{D}} \cdots \widetilde{\otimes}_{\mathfrak{D}} \mathfrak{E}(\mathbb{H})_{t_{n}}\right) \times$

$$
\begin{aligned}
& \times\left(\mathfrak{E}(\mathbb{H})_{t_{1}} \widetilde{\otimes}_{\mathfrak{D}} \cdots \widetilde{\otimes}_{\mathfrak{D}} \mathfrak{E}(\mathbb{H})_{t_{n}}\right) \rightarrow \mathbb{C}, d_{\varrho, S}(a, b):=\operatorname{tr}\left(C_{t_{0}}^{\prime}(\sqrt{a}) \varrho C_{t_{0}}^{\prime}(\sqrt{b})^{\dagger}\right) \text {, satisfies for all } a, b \in \\
& \mathfrak{E}(\mathbb{H})_{t_{1}} \widetilde{\otimes}_{\mathfrak{D}} \cdots \widetilde{\otimes}_{\mathfrak{D}} \mathfrak{E}(\mathbb{H})_{t_{n}} \\
& \text { - } d_{\varrho, S}(a, a) \in \mathbb{R} \text { and } d_{\varrho, S}(a, a) \geq 0 \\
& \text { - } d_{\varrho, S}(a, b)=d_{\varrho, S}(b, a)^{*} \\
& \text { - } d_{\varrho, S}(1,1)=1
\end{aligned}
$$


- $d_{\varrho, S}(0, a)=0$, for all $a$.

- $d_{\varrho, S}\left(a_{1} \widetilde{\oplus}_{\mathfrak{D}} a_{2}, b\right)=d_{\varrho, S}\left(a_{1}, b\right)+d_{\varrho, S}\left(a_{2}, b\right)$ for all $a_{1}, a_{2}, b \in \mathfrak{E}(\mathbb{H})_{t_{1}} \widetilde{\otimes}_{\mathfrak{D}} \cdots \widetilde{\otimes}_{\mathfrak{D}} \mathfrak{E}(\mathbb{H})_{t_{n}}$, for which the left hand side is well-defined.

Definition 20 Let $(\mathfrak{T}, \leq)$ be a partially ordered set. A $\mathfrak{T}$-DIRECTED SYSTEM of D-posets is a family $D_{\mathfrak{T}}:=\left\{D_{t}, t \in \mathfrak{T}\right\}$ of D-posets supplied with a family of morphisms $f_{t s}: D_{t} \rightarrow D_{s}, t, s \in$ $\mathfrak{T}$, defined iff $t \leq s$, such that

- $f_{t t}=i d_{D_{t}}$, for all $t \in \mathfrak{T}$;

- If $t \leq s \leq r$ in $\mathfrak{T}$, then $f_{s r} f_{t s}=f_{t r}$.

Let $D_{\mathfrak{T}}$ be a $\mathfrak{T}$-directed system of D-posets. Then a D-poset $\mathfrak{L}$ supplied with a family of morphisms $\left\{f_{t}: D_{t} \rightarrow \mathfrak{L}\right\}_{t \in \mathfrak{T}}$ is called the DIRECT LIMIT of $D_{\mathfrak{T}}$ if

- If $t \leq s$ in $\mathfrak{T}$, then $f_{s} f_{t s}=f_{t}$;

- If $D$ is a D-poset supplied with a set of morphisms $\left\{g_{t}: D_{t} \rightarrow D, t \in \mathfrak{T}\right\}$, then there exists a unique morphism $g: \mathfrak{L} \rightarrow D$, such that $g f_{t}=g_{t}$, for all $t \in \mathfrak{T}$.

The direct limit of a directed system of D-posets always exists (Pulmannová, 1995).

Let in the sequel $\mathfrak{T}$ denote the set of all finite subsets of $\mathbb{R}$ partially ordered by set inclusion. For every $t \in \mathbb{R}$ set $\mathfrak{E}(\mathbb{H})_{t}:=\mathfrak{E}(\mathbb{H})$ and for every $T=\left\{t_{1}, \ldots, t_{n}\right\} \in \mathfrak{T}$ set $\mathfrak{E}(\mathbb{H})_{T}:=\mathfrak{E}(\mathbb{H})_{t_{1}} \widetilde{\otimes}_{\mathfrak{D}}$. . $\widetilde{\otimes}_{\mathfrak{D}} \mathfrak{E}(\mathbb{H})_{t_{n}}$. Then it has been shown by Pulmannová (1995) that for every $T \subset S \in \mathfrak{T}$ there exists a morphism $f_{T S}: \mathfrak{E}(\mathbb{H})_{T} \rightarrow \mathfrak{E}(\mathbb{H})_{S}$ such that $\left\{\mathfrak{E}(\mathbb{H})_{T}, T \in \mathfrak{T}\right\}$ supplied with $\left\{f_{T S}, T \subset S \in \mathfrak{T}\right\}$ is a $\mathfrak{T}$-directed system. Let, e.g., $T=\left\{t_{1}, t_{3}\right\} \subset S=\left\{t_{1}, t_{2}, t_{3}\right\}$, then $f_{T S}\left(A \widetilde{\otimes}_{\mathfrak{D}} B\right)=A \widetilde{\otimes}_{\mathfrak{D}} 1 \tilde{\otimes}_{\mathfrak{D}} B$.

Therefore the direct limit of $\left\{\left(\mathfrak{E}(\mathbb{H})_{T}, \widetilde{\oplus}_{\mathfrak{D}}\right), T \in \mathfrak{T}\right\}$ exists and will be denoted by $\left(\mathfrak{E}(\mathbb{H})_{\mathfrak{T}}, \widetilde{\oplus}_{\mathfrak{D}}\right)$. $\mathfrak{E}(\mathbb{H})_{\mathfrak{T}}$ can be constructed as follows: consider the disjoint union $\cup_{T \in \mathfrak{T}} \mathfrak{E}(\mathbb{H})_{T}$ and call two elements $h_{1}, h_{2}$ of $\cup_{T \in \mathfrak{T}} \mathfrak{E}(\mathbb{H})_{T}$ EQUIVALENT if there exist $T_{1}, T_{2}, T_{12} \in \mathfrak{T}$ such that $h_{1} \in T_{1} \subset T_{12}$, $h_{2} \in T_{2} \subset T_{12}$ and such that $f_{T_{1} T_{12}}\left(h_{1}\right)=f_{T_{2} T_{12}}\left(h_{2}\right)$. Then $\mathfrak{E}(\mathbb{H})_{\mathfrak{T}}$ is the quotient space of $\cup_{T \in \mathfrak{T}} \mathfrak{E}(\mathbb{H})_{T}$ by the such defined equivalence relation. It is easy to extend the D-poset structures on $\mathfrak{E}(\mathbb{H})_{T}$, for $T \in \mathfrak{T}$ to a D-poset structure on $\mathfrak{E}(\mathbb{H})_{\mathfrak{T}}$. Thus the above defined decoherence functional can be generalized to a decoherence functional on $\mathfrak{E}(\mathbb{H})_{\mathfrak{T}} \times \mathfrak{E}(\mathbb{H})_{\mathfrak{T}}$ by

$$
d_{\varrho, \mathfrak{T}}: \mathfrak{E}(\mathbb{H})_{\mathfrak{T}} \times \mathfrak{E}(\mathbb{H})_{\mathfrak{T}} \rightarrow \mathbb{C}, d_{\varrho, \mathfrak{T}}(A, B):=d_{\varrho, \mathfrak{s}(A) \cup \mathfrak{s}(B)}\left(f_{\mathfrak{s}(A), \mathfrak{s}(A) \cup \mathfrak{s}(B)} A, f_{\mathfrak{s}(B), \mathfrak{s}(A) \cup \mathfrak{s}(B)} B\right),
$$

where $\mathfrak{s}(A)$ denotes the temporal support of $A$ and $\mathfrak{s}(B)$ denotes the temporal support of $B$ and $f_{\mathfrak{s}(A), \mathfrak{s}(A) \cup \mathfrak{s}(B)}$ and $f_{\mathfrak{s}(B), \mathfrak{s}(A) \cup \mathfrak{s}(B)}$ denote the canonical morphisms in the directed system $\left\{\mathfrak{E}(\mathbb{H})_{T}, T \in \mathfrak{T}\right\}$ from $\mathfrak{E}(\mathbb{H})_{\mathfrak{s}(A)}$ and from $\mathfrak{E}(\mathbb{H})_{\mathfrak{s}(B)}$ to $\mathfrak{E}(\mathbb{H})_{\mathfrak{s}(A) \cup \mathfrak{s}(B)}$ respectively.

Lemma 8 The so defined decoherence functional $d_{\varrho, \mathfrak{T}}$ satisfies all properties listed in Theorem 3; in particular, $d_{\varrho, \mathfrak{T}}\left(a_{1} \widetilde{\oplus}_{\mathfrak{D}} a_{2}, b\right)=d_{\varrho, \mathfrak{T}}\left(a_{1}, b\right)+d_{\varrho, \mathfrak{T}}\left(a_{2}, b\right)$ for all $a_{1}, a_{2}, b \in \mathfrak{E}(\mathbb{H})_{\mathfrak{T}}$ for which the left hand side is well-defined. 
Now we are ready to formulate the consistency conditions in our generalized history formalism.

Definition 21 Let $\mathcal{G}$ be a set of elements of $\left(\mathfrak{E}(\mathbb{H})_{\mathfrak{T}}, \widetilde{\oplus}_{\mathfrak{D}}\right)$. Then an arbitrary subset $\Delta \mathcal{G}$ of $\widetilde{\oplus}_{\mathfrak{D}} \mathcal{G}$ is said to be PRECONSISTENT WITH RESPECT TO THE STATE $\varrho$ if

$\operatorname{Re} d_{\varrho, \mathfrak{T}}(a, b)=0$, for all $a, b \in \Delta \mathcal{G}$ for which $a \widetilde{\oplus}_{\mathfrak{D}} b$ is well-defined in $\widetilde{\oplus}_{\mathfrak{D}} \mathcal{G}$.

Further, $\widetilde{\oplus}_{\mathfrak{D}} \mathcal{G}$ is said to be CONSISTENT WITH RESPECT TO THE STATE $\varrho$ if

$\operatorname{Re} d_{\varrho, \mathfrak{T}}(a, b)=0$, for all $a, b \in \widetilde{\oplus}_{\mathfrak{D}} \mathcal{G}$ for which $a \widetilde{\oplus}_{\mathfrak{D}} b$ is well-defined.

An arbitrary subset $\mathcal{G}_{0}$ of $\mathfrak{E}(\mathbb{H})_{\mathfrak{T}}$ is said to be CONSISTENT W.R.T. $\varrho$ if there exists a subset $\mathcal{G}$ of $\mathfrak{E}(\mathbb{H})_{\mathfrak{T}}$ such that $\mathcal{G}_{0}=\widetilde{\oplus}_{\mathfrak{D}} \mathcal{G}$ is consistent w.r.t $\varrho$.

Remark 13 Above we have found two sorts of D-posets on which the decoherence functional can be unambiguously defined such that the decoherence functional is additive in both arguments. The decoherence functional induces a probability measure on consistent sub-D-posets of $\left(\mathfrak{E}(\mathbb{H})_{\mathfrak{T}}, \widetilde{\oplus}_{\mathfrak{D}}\right)$ and on full D-posets of effect histories. Since effect histories represent the general physical properties of a quantum system, for a physical description of a quantum system one needs a probability measure which is defined directly on some set of effect histories. This task has only be achieved in the latter case for the full D-posets of effect histories. The elements of $\mathfrak{E}(\mathbb{H})_{\mathfrak{T}}$ are not effect histories. Hence in the former case we have to single out those sets of effect histories for which the description in terms of elements of consistent sub-D-posets of $\left(\mathfrak{E}(\mathbb{H})_{\mathfrak{T}}, \widetilde{\oplus}_{\mathfrak{D}}\right)$ can be lifted to an unambiguous description in terms of effect histories. Furthermore, it is possible to define a 'reasoning' on a D-poset $D$ on which an additive decoherence functional $d$ is given. Let $a, b, c \in(D, \oplus)$ be pairwise orthogonal elements, then we say that $a \oplus c \Longrightarrow_{d} a \oplus b$ if $d(a \oplus c, a \oplus c)=d(a, a) \neq 0$ and $d(a \oplus b, a \oplus b)=d(a \oplus b \oplus c, a \oplus b \oplus c) \neq 0$. If $d$ induces a probability measure on $D$, then the second condition is redundant. Hence we also have to single out those sets of effect histories for which the reasoning in terms of elements of consistent sub-D-posets of $\left(\mathfrak{E}(\mathbb{H})_{\mathfrak{T}}, \widetilde{\oplus}_{\mathfrak{D}}\right)$ can be lifted to an unambiguous reasoning in terms of effect histories.

Remark 14 If $\widetilde{\oplus}_{\mathfrak{D}} \mathcal{G}$ is consistent w.r.t. $\varrho$, then $d_{\varrho, \mathfrak{T}}(a, a)$ can be interpreted as probability of a in $\widetilde{\oplus}_{\mathfrak{D}} \mathcal{G}$.

Remark 15 There is a canonical map $\mathfrak{N}: \mathbb{E}_{\text {fin }}(\mathbb{H}) \rightarrow \mathfrak{E}(\mathbb{H})_{\mathfrak{T}}$ defined by $\mathfrak{N}(u):=\widetilde{\otimes}_{\mathfrak{D}, t \in \mathfrak{s}(u)} u_{t}$. The decoherence functional $d_{\varrho, \mathfrak{T}}$ induces a map $d_{\varrho, \mathfrak{N}}: \mathbb{E}_{\text {fin }}(\mathbb{H}) \times \mathbb{E}_{f i n}(\mathbb{H}) \rightarrow \mathbb{C}, d_{\varrho, \mathfrak{N}}\left(p_{1}, p_{2}\right):=$ $d_{\varrho, \mathfrak{T}}\left(\mathfrak{N}\left(p_{1}\right), \mathfrak{N}\left(p_{2}\right)\right)$.

Definition 22 We say that a map $\mathfrak{F}: \Delta \subset \widehat{\mathbb{E}}(\mathbb{H}) \rightarrow \mathfrak{E}(\mathbb{H})_{\mathfrak{T}}$ EXTENDS $\mathfrak{N}$ if $\mathfrak{F}(p)=\mathfrak{N}(p)$, for all $p \in \Delta \cap \mathbb{E}_{\text {fin }}(\mathbb{H})$. 
Definition 23 A Boolean lattice $\left(\mathcal{B}, \vee_{\mathcal{B}}, \wedge_{\mathcal{B}}, \neg_{\mathcal{B}}\right)$ is said to be an ALlOWED BooleAn LATTICE OF (INHOMOGENEOUS) EFFECT HISTORIES if the following conditions are satisfied

- $\mathcal{B}$ is a Boolean sublattice of $\widehat{\mathbb{E}}_{f i n}(\mathbb{H})$; the lattice-operations $\vee_{\mathcal{B}}$ and $\wedge_{\mathcal{B}}$ are the restrictions of $\vee_{\mathcal{L}}$ and $\wedge_{\mathcal{L}}$ to $\mathcal{B}$ respectively. The lattice-operations $\vee_{\mathcal{B}}$ and $\wedge_{\mathcal{B}}$ are such that a complementation $\neg \mathcal{B}$ can be unambiguously defined on $\mathcal{B}$;

- $\mathcal{B}$ is atomic and the set of atoms consists of homogeneous elements;

- The canonical map $\mathfrak{N}: \mathcal{B} \cap \mathbb{E}_{\text {fin }}(\mathbb{H}) \rightarrow \mathfrak{E}(\mathbb{H})_{\mathfrak{T}}$ defined in Remark 15 can be uniquely extended to a positive valuation $\mathrm{B}$ on $\mathcal{B}$ with values in $\mathfrak{E}(\mathbb{H})_{\mathfrak{T}}$, i.e., to a map $\mathrm{B}: \mathcal{B} \rightarrow \mathfrak{E}(\mathbb{H})_{\mathfrak{T}}$ satisfying $\mathrm{B}\left(b_{1} \vee_{\mathcal{B}} b_{2}\right) \widetilde{\ominus}_{\mathfrak{D}} \mathrm{B}\left(b_{1}\right)=\mathrm{B}\left(b_{2}\right) \tilde{\ominus}_{\mathfrak{D}} \mathrm{B}\left(b_{1} \wedge_{\mathcal{B}} b_{2}\right)$, for all $b_{1}, b_{2} \in \mathcal{B}$. This condition means in particular that the left hand side and the right hand side are well-defined for all $b_{1}, b_{2} \in \mathcal{B}$.

An allowed Boolean sublattice of $\widehat{\mathbb{E}}_{\text {fin }}(\mathbb{H})$ will be briefly denoted by $(\mathcal{B}, \mathrm{B})$.

Remark 16 The greatest element $1_{\mathcal{B}}$ and the least element $0_{\mathcal{B}}$ in $\mathcal{B}$ do not necessarily coincide with the greatest element 1 and the least element 0 in $\widehat{\mathbb{E}}_{f i n}(\mathbb{H})$ respectively. If the set of atoms of $\mathcal{B}$ contains more than two different elements, then $0_{\mathcal{B}}=0$. Every inhomogeneous history in $\mathcal{B}$ is the join of disjoint homogeneous atoms of $\mathcal{B}$.

Remark 17 The decoherence functional $d_{\varrho, \mathfrak{T}}$ induces a decoherence functional on $\mathcal{B} \times \mathcal{B}$ by $d_{\varrho, \mathcal{B}}: \mathcal{B} \times \mathcal{B} \rightarrow \mathbb{C}, d_{\varrho, \mathcal{B}}\left(p_{1}, p_{2}\right):=d_{\varrho, \mathfrak{T}}\left(\mathrm{B}\left(p_{1}\right), \mathrm{B}\left(p_{2}\right)\right)$, which is additive in both arguments with respect to the Boolean lattice structure on $\mathcal{B}$.

Lemma 9 The value $\mathrm{B}(u)$ does not depend on the choice of the allowed Boolean lattice of effect histories $\mathcal{B} \ni u$.

Proof: If $u$ is a homogeneous history, then the assertion is trivial. If $u$ is an inhomogeneous history belonging to two allowed Boolean lattices, say $(\mathcal{B}, \mathrm{B})$ and $\left(\mathcal{B}^{\prime}, \mathrm{B}^{\prime}\right)$, then it is easy to see that there exist homogeneous histories $h_{1}, \ldots, h_{n} \in \mathcal{B} \cap \mathcal{B}^{\prime}$ (not necessarily atoms of $\mathcal{B}$ and $\mathcal{B}^{\prime}$ ) such that $u=\bigvee_{i=1}^{n} h_{i}$. Thus $\mathrm{B}(u)=\mathrm{B}^{\prime}(u)$.

Definition 24 Let $u_{1}$ and $u_{2}$ denote two finite effect histories. Then we say that $u_{1}$ IMPLIES $u_{2}$ IN THE STATE $\varrho$ if $u_{1}$ and $u_{2}$ lie in a common allowed Boolean lattice $(\mathcal{B}, \mathrm{B})$ of effect histories and if $d_{\varrho, \mathcal{B}}\left(u_{1} \wedge_{\mathcal{B}} u_{2}, u_{1} \wedge_{\mathcal{B}} u_{2}\right)=d_{\varrho, \mathcal{B}}\left(u_{1}, u_{1}\right) \neq 0$ and if $d_{\varrho, \mathcal{B}}\left(u_{1} \vee_{\mathcal{B}} u_{2}, u_{1} \vee_{\mathcal{B}} u_{2}\right)=d_{\varrho, \mathcal{B}}\left(u_{2}, u_{2}\right) \neq 0$ In this case we write $u_{1} \Longrightarrow u_{2}$.

Remark 18 It is easy to verify that if $p_{1} \Longrightarrow p_{2}$ is valid in one allowed Boolean lattice, then $p_{1} \Longrightarrow p_{2}$ is also valid in every other allowed Boolean lattice of $\widehat{\mathbb{E}}_{f i n}(\mathbb{H})$ containing $p_{1}$ and $p_{2}$. 
Example: If $u_{1}$ and $u_{2}$ are nonzero finite homogeneous effect histories such that $u_{1} \leq u_{2}$ where $\leq$ denotes the partial order defined in Remark 10, then $u_{1} \Longrightarrow \varrho u_{2}$ for all $\varrho$ for which $d_{\varrho, \mathfrak{T}}\left(u_{1}, u_{1}\right) \neq 0$ and $d_{\varrho, \mathfrak{T}}\left(u_{2}, u_{2}\right) \neq 0$. To see this, consider the trivial allowed Boolean lattice $\mathcal{B}_{0}$ containing only the two elements $u_{1} \equiv 0_{\mathcal{B}_{0}}$ and $u_{2} \equiv 1_{\mathcal{B}_{0}}$.

Remark 19 Let $h_{1}$ and $h_{2}$ denote two nonzero ordinary homogeneous histories. If $h_{1} \Longrightarrow_{\varrho} h_{2}$ is valid for some $\varrho$ in the sense of Section 3 , then $h_{1} \Longrightarrow_{\varrho} h_{2}$ is also valid in the sense of Definition 24 and vice versa. To see this, consider the allowed Boolean lattice $\mathcal{B}$ of effect histories consisting of the effect histories $h_{1} ; h_{2} ; h_{1} \vee_{\mathcal{B}} h_{2} ; h_{1} \wedge_{\mathcal{B}} h_{2} . \quad\left(h_{1} \vee_{\mathcal{B}} h_{2}\right.$ is a possibly inhomogeneous effect history. It is important not to confuse the notion of ordinary inhomogeneous history as defined in Section 2 and the notion of inhomogeneous effect history as defined in this section. In this section the term 'inhomogeneous history' is always meant in the sense of Definition 13 (unless explicitly otherwise stated).) The least element in $\mathcal{B}$ is $h_{1} \wedge_{\mathcal{B}} h_{2}$ and the greatest element in $\mathcal{B}$ is $h_{1} \vee_{\mathcal{B}} h_{2}$. The atoms in $\mathcal{B}$ are $h_{1}$ and $h_{2}$. The map $\mathrm{B}$ extends $\mathfrak{N}$ and hence maps homogeneous histories to their corresponding projection operators on $\otimes_{i} \mathbb{H}_{i}$ which in turn can be identified with the corresponding homogeneous elements in $\mathfrak{E}(\mathbb{H})_{\mathfrak{T}}$, e.g., $h_{1}$ is mapped to $\mathrm{B}\left(h_{1}\right)=\otimes_{t \in \mathfrak{s}\left(h_{1}\right)} h_{1, t}$. The condition $\mathrm{B}\left(b_{1} \vee_{\mathcal{B}} b_{2}\right) \tilde{\ominus}_{\mathfrak{D}} \mathrm{B}\left(b_{1}\right)=\mathrm{B}\left(b_{2}\right) \tilde{\ominus}_{\mathfrak{D}} \mathrm{B}\left(b_{1} \wedge_{\mathcal{B}} b_{2}\right)$, for all $b_{1}, b_{2} \in \mathcal{B}$ fixes $\mathrm{B}$ on the inhomogeneous element $h_{1} \vee_{\mathcal{B}} h_{2}$ of $\mathcal{B}$.

On the other hand consider the set $\mathcal{C} \subset \mathcal{P}_{\text {fin }}^{\otimes}(\mathbb{H})$ consisting of $\left\{h_{1}, h_{2}, h_{1} \vee h_{2}, h_{1} \wedge h_{2}\right\}$ where $h_{1} \vee h_{2}$ and $h_{1} \wedge h_{2}$ are ordinary histories (possibly inhomogeneous in the sense of Section 2). The assertion of this remark is now an easy consequence of Definition 24 and Corollary 1.

Definition 25 An allowed Boolean lattice $(\mathcal{B}, \mathrm{B})$ is called CONSISTEnT W.R.T. $\varrho$ if $\mathrm{B}(\mathcal{B})$ is preconsistent w.r.t. $\varrho$.

Theorem 4 Let $(\mathcal{B}$, B) be a consistent allowed Boolean lattice of effect histories. Then the decoherence functional $d_{\varrho, \mathfrak{T}}$ induces a probability functional on $\mathcal{B}$ by $b \mapsto \frac{d_{\varrho, \mathfrak{T}}(\mathrm{B}(b), \mathrm{B}(b))}{d_{\varrho, \mathfrak{T}}\left(\mathrm{B}\left(1_{\mathcal{B}}\right), \mathrm{B}\left(1_{\mathcal{B}}\right)\right)}$.

Before formulating the generalized logical rule of interpretation we return briefly to the discussion of exceptional sets of effect histories. We have already seen above that the D-poset structure on $\mathfrak{E}(\mathbb{H})$ is not unique. It is possible to define a countably infinite family of D-poset structures on $\mathfrak{E}(\mathbb{H})$. Let $\alpha$ be a rational number with $\alpha>0$ and define

$$
A \oplus_{\alpha} B:=\left(A^{1 / \alpha}+B^{1 / \alpha}\right)^{\alpha}, \text { for all } A, B \in \mathfrak{E}(\mathbb{H}) \text { satisfying } A^{1 / \alpha}+B^{1 / \alpha} \leq 1 .
$$

That these expressions are well-defined is a consequence of the work of Langer (1962). In particular it follows from Proposition 2 in (Langer, 1962) that $E^{\alpha}$ is well-defined and that $E^{\alpha}$ is itself an effect operator for all $E \in \mathfrak{E}(\mathbb{H})$ and all $\alpha \in \mathbb{Q}, \alpha>0$. The pair $\left(\mathfrak{E}(\mathbb{H}), \oplus_{\alpha}\right)$ is a D-poset for every $\alpha>0$. Clearly, $\oplus_{1}=\oplus$ and $\oplus_{2}=\widetilde{\oplus}$. Moreover, $\left(\mathfrak{E}(\mathbb{H}), \oplus_{\alpha}\right)$ is an $\mathbb{N}_{\alpha^{-}}$ semimodule. It is now possible to define the tensor product $E_{1} \widehat{\otimes}_{\alpha, \mathfrak{D}} \cdots \widehat{\otimes}_{\alpha, \mathfrak{D}} E_{m}$ in the category of $\mathbb{N}_{\alpha}$-semimodules to be the D-poset consisting of all $\sim_{\alpha, \mathfrak{D}}$-equivalence classes of $\oplus_{\alpha, \mathfrak{D}}$-parts of $1 \otimes_{\mathbb{N}} \cdots \otimes_{\mathbb{N}} 1$ subject to the familiar three relations. The equivalence relation $\sim_{\alpha, \mathfrak{D}}$ and the 
partial addition $\oplus_{\alpha, \mathfrak{D}}$ are defined completely analogously to $\sim_{\mathfrak{D}}$ and $\oplus_{\mathfrak{D}}$.

Pick an arbitrary finite homogeneous effect history $w_{0}$, choose $k \in \mathbb{N}, k>0$ and choose for all $r \in\{1, \ldots, m\}$ a $k$-tuple of times $\left(t_{r, 1}^{*}, t_{r, 2}^{*}, \ldots, t_{r, k}^{*}\right)$ with $t_{r, k}^{*}>\ldots>t_{r, 2}^{*}>t_{r, 1}^{*}$ such that for all $r$ there is no $t \in \mathfrak{s}\left(w_{0}\right)$ such that $t_{r, l}^{*} \geq t \geq t_{r, l-1}^{*}$ for some $1<l \leq k$. Now we pick $m$ effect operators $E_{1}, \ldots, E_{m}$ and define $\sqrt{E_{r}(t)}:=U\left(t, t_{i}\left(w_{E_{1}, \ldots, E_{m}}\right)\right) \sqrt{E_{r}} U\left(t, t_{i}\left(w_{E_{1}, \ldots, E_{m}}\right)\right)^{\dagger}$. Here $t_{i}\left(w_{E_{1}, \ldots, E_{m}}\right)$ denotes the initial time of the effect history $w_{E_{1}, E_{2}, \ldots, E_{m}}$ defined by $\mathfrak{s}_{m, k}^{*}\left(w_{0}\right):=\mathfrak{s}\left(w_{E_{1}, \ldots, E_{m}}\right):=\mathfrak{s}\left(w_{0}\right) \cup\left\{t_{1,1}^{*}, \ldots, t_{1, k}^{*}, \ldots, t_{m, 1}^{*}, \ldots, t_{m, k}^{*}\right\}$ and

$$
\left(w_{E_{1}, \ldots, E_{m}}\right)_{t}:=\left\{\begin{array}{lll}
\left(w_{0}\right)_{t} & : & t \neq t_{r, 1}^{*}, \ldots, t_{r, k}^{*} \\
E_{r}(t) & : & t \in\left\{t_{r, 1}^{*}, \ldots, t_{r, k}^{*}\right\}
\end{array} .\right.
$$

That is, $w_{E_{1}, \ldots, E_{m}}$ is the extension of $w_{0}$ by the effects $E_{r}(t)$ at the intermediate times $t_{r, j}^{*}$, where $r \in\{1, \ldots, m\}$ and $j \in\{1, \ldots, k\}$.

Define $\widehat{\mathfrak{E}}_{k, m}:=\left\{w_{E_{1}, \ldots, E_{m}} \mid E_{1}, \ldots, E_{m} \in \mathfrak{E}(\mathbb{H})\right\}$. The above decoherence functional $\widehat{d}_{\varrho, \mathfrak{s}_{m, k}^{*}}\left(w_{0}\right)$ restricted to the set $\Theta\left(\widehat{\mathfrak{E}}_{k, m}\right):=\left\{\Theta\left(w_{E_{1}, \ldots, E_{m}}\right) \mid E_{1}, \ldots, E_{m} \in \mathfrak{E}(\mathbb{H})\right\}$ is additive in both arguments, e.g.,

$$
\begin{aligned}
& \widehat{d}_{\varrho, \mathfrak{s}_{m, k}^{*}\left(w_{0}\right)}\left(\Theta\left(w_{E_{1} \oplus_{2 / k} D_{1}, \ldots, E_{m}}\right), \Theta\left(w_{F_{1}, \ldots, F_{m}}\right)\right)= \widehat{d}_{\varrho, \mathfrak{s}_{m, k}^{*}\left(w_{0}\right)}\left(\Theta\left(w_{E_{1}, \ldots, E_{m}}\right), \Theta\left(w_{F_{1}, \ldots, F_{m}}\right)\right) \\
&+\widehat{d}_{\varrho, \mathfrak{s}_{m, k}^{*}}\left(w_{0}\right) \\
&\left(\Theta\left(w_{D_{1}, \ldots, E_{m}}\right), \Theta\left(w_{F_{1}, \ldots, F_{m}}\right)\right),
\end{aligned}
$$

for arbitrary $E_{1}, \ldots, E_{m}, D_{1}, F_{1}, \ldots, F_{m} \in \mathfrak{E}(\mathbb{H})$ for which $E_{1} \oplus_{2 / k} D_{1}$ is well-defined. $\Theta\left(w_{E_{1}, \ldots, E_{m}}\right)$ depends upon $E_{1}, . ., E_{m}$ only through the tensor product $E_{1} \widehat{\otimes}_{2 / k, \mathfrak{D}} \cdots \widehat{\otimes}_{2 / k, \mathfrak{D}} E_{m}$ and hence there is an embedding $\mathfrak{b}_{k}$ mapping $\Theta\left(\widehat{\mathfrak{E}}_{m, k}\right)$ injectively to $\mathfrak{E}\left(\mathbb{H}_{1}\right) \widehat{\otimes}_{2 / k, \mathfrak{D}} \cdots \widehat{\otimes}_{2 / k, \mathfrak{D}} \mathfrak{E}\left(\mathbb{H}_{m}\right)$. Thus $\Theta\left(\widehat{\mathfrak{E}}_{m, k}\right)$ can be identified with the set of all homogeneous elements in $\mathfrak{E}\left(\mathbb{H}_{1}\right) \widehat{\otimes}_{2 / k, \mathfrak{D}} \cdots \widehat{\otimes}_{2 / k, \mathfrak{D}} \mathfrak{E}\left(\mathbb{H}_{m}\right)$, i.e., elements of the form $E_{1} \widehat{\otimes}_{2 / k, \mathfrak{D}} \cdots \widehat{\otimes}_{2 / k, \mathfrak{D}} E_{m}$. In this sense $\mathfrak{E}\left(\mathbb{H}_{1}\right) \widehat{\otimes}_{2 / k, \mathfrak{D}} \cdots \widehat{\otimes}_{2 / k, \mathfrak{D}} \mathfrak{E}\left(\mathbb{H}_{m}\right)$

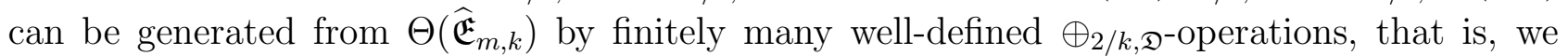
write $\bigoplus_{2 / k, \mathfrak{D}} \mathfrak{b}_{k}\left(\Theta\left(\widehat{\mathfrak{E}}_{m, k}\right)\right) / \sim_{2 / k, \mathfrak{D}}=\mathfrak{E}\left(\mathbb{H}_{1}\right) \widehat{\otimes}_{2 / k, \mathfrak{D}} \cdots \widehat{\otimes}_{2 / k, \mathfrak{D}} \mathfrak{E}\left(\mathbb{H}_{m}\right)$. The decoherence functional $\widehat{d}_{\varrho, \mathfrak{s}_{m, k}^{*}\left(w_{0}\right)}$ induces a decoherence functional $d_{\varrho, \mathfrak{s}_{m, k}^{*}}^{\mathfrak{b}_{k}\left(w_{0}\right)}$ on $\mathfrak{b}_{k}\left(\Theta\left(\widehat{\mathfrak{E}}_{m, k}\right)\right)$ in a trivial way by

$$
d_{\varrho, \mathfrak{s}_{m, k}^{*}\left(w_{0}\right)}^{\mathfrak{b}_{k}}(a, b):=\widehat{d}_{\varrho, \mathfrak{s}_{m, k}^{*}\left(w_{0}\right)}\left(\mathfrak{b}_{k}^{-1}(a), \mathfrak{b}_{k}^{-1}(b)\right) .
$$

The right hand side is well-defined since $\mathfrak{b}_{k}$ maps $\Theta\left(\widehat{\mathfrak{E}}_{m, k}\right)$ bijectively to $\mathfrak{b}_{k}\left(\Theta\left(\widehat{\mathfrak{E}}_{m, k}\right)\right)$. It is now easy to see that for arbitrary $m \in \mathbb{N}$ the decoherence functional $d_{\varrho, \mathfrak{s}_{m, k}^{*}}^{\mathfrak{b}_{k}}\left(w_{0}\right)$ can be extended to a $\oplus_{2 / k, \mathfrak{D}}$-additive functional on the D-poset $\bigoplus_{2 / k, \mathfrak{D}} \mathfrak{b}_{k}\left(\Theta\left(\widehat{\mathfrak{E}}_{m, k}\right)\right) / \sim_{2 / k, \mathfrak{D}}$.

Let $\mathcal{B}_{k}$ be a subset of the free lattice $\mathcal{L}\left(\widehat{\mathfrak{E}}_{m, k}\right)$ generated by $\widehat{\mathfrak{E}}_{m, k}$ (for $m \in \mathbb{N}, m>0$ ). We will say that $\widehat{\mathcal{B}}_{k}:=\zeta\left(\mathcal{B}_{k}\right)$ is an ALLOWED BoOLEAN LATTICE OF (INHOMOGENEOUS) EFFECT HISTORIES OF ORDER $k$ if conditions analogous to those in Definition 23 are satisfied, namely

- $\widehat{\mathcal{B}}_{k}$ is a Boolean sublattice of $\mathcal{L}\left(\widehat{\mathfrak{E}}_{m, k}\right) / \sim_{\mathbb{E}}$

- $\widehat{\mathcal{B}}_{k}$ is atomic and the set of atoms consists of homogeneous elements; 
- the map $\mathfrak{b}_{k} \circ \Theta$ can be uniquely extended to a positive valuation $\mathfrak{I}_{k}$ on $\widehat{\mathcal{B}}_{k}$ with values in $\bigoplus_{2 / k, \mathfrak{D}} \mathfrak{b}_{k}\left(\Theta\left(\widehat{\mathfrak{E}}_{m, k}\right)\right) / \sim_{2 / k, \mathfrak{D}}$, i.e., to a map $\mathfrak{I}_{k}: \widehat{\mathcal{B}}_{k} \rightarrow \bigoplus_{2 / k, \mathfrak{D}} \mathfrak{b}_{k}\left(\Theta\left(\widehat{\mathfrak{E}}_{m, k}\right)\right) / \sim_{2 / k, \mathfrak{D}}$ satisfying $\mathfrak{I}_{k}\left(d_{1} \vee_{k} d_{2}\right) \ominus_{2 / k, \mathfrak{D}} \mathfrak{I}_{k}\left(d_{1}\right)=\mathfrak{I}_{k}\left(d_{2}\right) \ominus_{2 / k, \mathfrak{D}} \mathfrak{I}_{k}\left(d_{1} \wedge_{k} d_{2}\right)$ for $d_{1}, d_{2} \in \widehat{\mathcal{B}}_{k}$. This condition means in particular that the left hand side and the right hand side are both well-defined for all $d_{1}, d_{2} \in \widehat{\mathcal{B}}_{k}$. Here $\wedge_{k}$ and $\vee_{k}$ denote the lattice-operations on $\widehat{\mathcal{B}}_{k}$.

The decoherence functional $d_{\varrho, \mathfrak{s}_{m, k}^{*}}^{\mathfrak{b}_{k}}\left(w_{0}\right)$ and the map $\mathfrak{I}_{k}$ induce a decoherence functional on $\widehat{\mathcal{B}}_{k} \times \widehat{\mathcal{B}}_{k}$ by

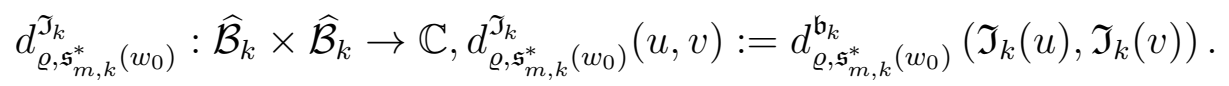

The decoherence functional $d_{\varrho, \mathfrak{s}_{m, k}^{*}}^{\Im_{k}}\left(w_{0}\right)$ is additive in both arguments with respect to the Boolean lattice structure on $\widehat{\mathcal{B}}_{k}$. Furthermore, we say that an allowed Boolean lattice $\widehat{\mathcal{B}}_{k}$ of effect histories of order $k$ is a CONSISTENT ALLOWED BOOLEAN LATTICE OF EFFECT HISTORIES OF ORDER $k$ if the decoherence functional $d_{\varrho, \mathfrak{s}_{m, k}^{*}}^{\mathfrak{I}_{k}}\left(w_{0}\right)$ defines a probability measure on the Boolean lattice $\widehat{\mathcal{B}}_{k}$ by

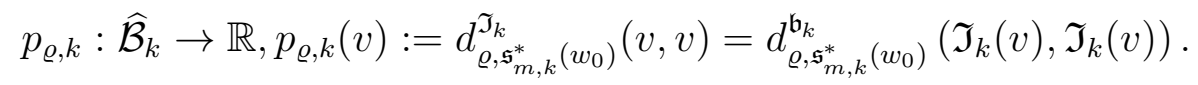

It is clear that this $p_{\varrho, k}$ defines a probability measure on $\widehat{\mathcal{B}}_{k}$ if and only if $\operatorname{Re} d_{\varrho, \mathfrak{s}_{m, k}^{*}\left(w_{0}\right)}^{\mathfrak{I}_{k}}(u, v)=$ $\operatorname{Re} d_{\varrho, \mathfrak{s}_{m, k}^{*}\left(w_{0}\right)}^{\mathfrak{b}_{k}}\left(\mathfrak{I}_{k}(u), \mathfrak{I}_{k}(v)\right)=0$, for all $u, v \in \widehat{\mathcal{B}}_{k}$ for which $\mathfrak{I}_{k}(u) \oplus_{2 / k, \mathfrak{D}} \mathfrak{I}_{k}(v)$ is well-defined.

Remark 20 It is possible to define the direct limit $\left(\mathfrak{E}(\mathbb{H})_{2 / k, \mathfrak{T}}, \oplus_{2 / k, \mathfrak{D}}\right)$ for arbitrary $k>0$ and fixed $w_{0}$ and to extend the decoherence functional $d_{\varrho, \mathfrak{s}_{m, k}^{*}}^{\mathfrak{\Im}_{k}}\left(w_{0}\right)$ to a decoherence functional on $\mathfrak{E}(\mathbb{H})_{2 / k, \mathfrak{T}}$. We omit the details.

Remark 21 The implication $\Longrightarrow \varrho$ has been defined above in Definition 24 only for pairs of effect histories belonging to an allowed Boolean lattice of effect histories (defined with respect to $\left.\left(\mathfrak{E}(\mathbb{H})_{\mathfrak{T}}, \widetilde{\oplus}_{\mathfrak{D}}\right)\right)$.

- Analogously we say for two effect histories $w_{1}$ and $w_{2}$ belonging to some common allowed Boolean lattice $\widehat{\mathcal{B}}_{k}$ of effect histories of order $k>0$ that $w_{1}$ IMPLIES $w_{2}$ IN THE STATE $\varrho$ if $d_{\varrho, \mathfrak{s}_{m, k}^{*}\left(w_{0}\right)}^{\Im_{k}}\left(w_{1} \wedge_{k} w_{2}, w_{1} \wedge_{k} w_{2}\right)=d_{\varrho, \mathfrak{s}_{m, k}^{*}\left(w_{0}\right)}^{\Im_{k}}\left(w_{1}, w_{1}\right) \neq 0$ and if $d_{\varrho, \mathfrak{s}_{m, k}^{*}\left(w_{0}\right)}^{\Im_{k}}\left(w_{1} \vee_{k} w_{2}, w_{1} \vee_{k} w_{2}\right)=$ $d_{\varrho, \mathfrak{s}_{m, k}^{*}\left(w_{0}\right)}^{\mathfrak{\Im}_{k}}\left(w_{2}, w_{2}\right) \neq 0$. We write $w_{1} \Longrightarrow{ }_{\varrho} w_{2}$.

- For two histories $u_{1}$ and $u_{2}$ belonging to a full set $\widetilde{\mathfrak{E}}$ of effect histories, we say that $u_{1}$ IMPLIES $u_{2}$ IN THE STATE $\varrho$ if $u_{1} \wedge u_{2}$ is well-defined in $\widetilde{\mathfrak{E}}$ and if $p_{\varrho}\left(u_{1} \wedge u_{2}\right)=p_{\varrho}\left(u_{1}\right) \neq 0$. Here $\wedge$ denotes the partially defined meet operation on $\widetilde{\mathfrak{E}}$ induced by the partially defined meet operation on $\mathbb{E}_{\text {fin }}(\mathbb{H})$. We write $u_{1} \Longrightarrow u_{2}$.

The universal rule of interpretation of quantum mechanics can now be generalized 
Rule 2 Propositions about quantum mechanical systems should solely be expressed in terms of inhomogeneous effect histories which represent the general physical properties of a quantum mechanical system.

- Every description of a quantum mechanical system (i.e., probabilistic or predictive statements) should be expressed either

- solely in terms of effect histories belonging to a common consistent allowed Boolean lattice $(\mathcal{B}, \mathrm{B})$ of effect histories. The probability measure on $\mathcal{B}$ is induced by the decoherence functional $d_{\varrho, \mathfrak{T}}$ on $\mathcal{B}$;

or

- solely in terms of a consistent allowed Boolean lattice $\left(\widehat{\mathcal{B}}_{k}, \mathfrak{I}_{k}\right)$ of effect histories of order $k>1$. The probability measure on $\widehat{\mathcal{B}}_{k}$ is defined by Equation 20;

or

- solely in terms of effect histories belonging to a full D-poset $\widetilde{\mathfrak{E}}$ of effect histories. The probability measure on $\widetilde{\mathfrak{E}}$ is defined by Equation 15.

- Every reasoning relates solely effect histories

- belonging to a common allowed Boolean lattice $(\mathcal{B}, \mathrm{B})$ of effect histories;

or

- belonging to a common allowed Boolean lattice $\left(\widehat{\mathcal{B}}_{k}, \mathfrak{I}_{k}\right)$ of effect histories of order $k>1$;

or

- belonging to a full D-poset of effect histories.

Every reasoning relating histories belonging to a common allowed Boolean lattice (of order 0 ) should solely be expressed in terms of the logical relations induced by the functional $d_{\varrho, \mathcal{B}}: \mathcal{B} \times \mathcal{B} \rightarrow \mathbb{C}$ defined in Remark 17 and Definition 24.

Every reasoning relating histories belonging to a common allowed Boolean lattice of order $k>1$ should solely be expressed in terms of the logical relations induced by the functional $d_{\varrho, \mathfrak{s}_{m, k}^{*}\left(w_{0}\right)}^{\Im_{k}}$ defined in Equation 19 (see also Remark 21).

Every reasoning relating histories belonging to a full D-poset of effect histories should solely be expressed in terms of the logical relations induced by the probability functional defined in Equation 15 (see also Remark 21).

Remark 22 It is easy to verify that if $p_{1} \Longrightarrow p_{2}$ is valid in one allowed Boolean lattice, then $p_{1} \Longrightarrow p_{2}$ is also valid in every other allowed Boolean lattice of $\widehat{\mathbb{E}}_{f i n}(\mathbb{H})$ containing $p_{1}$ and $p_{2}$. 


\subsection{The algebraic structure of generalized history theories}

We now summarize our discussion by stating the general axioms for a generalized quantum theory based on our generalized history concept. This subsection parallels the discussion in (Isham, 1994). However, it contains only a rough summary of the main concepts and structures. In every particular history theory one has to show that everything is well-defined and consistent and if necessary to modify the concepts and structures.

1. The space $\mathcal{U}$ of history filters or homogeneous histories.

- $\mathcal{U}$ is the space of the basic physical properties of a physical system. An element of $\mathcal{U}$ represents the equivalence classes of (operationally undistinguishable) basic entities (propositions) in the interpretation of the theory. There exists a map $F$ mapping the elements of $\mathcal{U}$ to a D-poset $\mathfrak{E}$. $\mathfrak{E}$ can be interpreted as the set of (equivalence classes of) one-time propositions. Moreover, there is a canonical map $\mathfrak{N}$ imbedding $\mathcal{U}$ in a D-poset $(\mathfrak{M}, \oplus)$. [in Section 4.3: $\mathcal{U}$ equals the space of homogeneous effect histories $\mathcal{U}=\mathbb{E}_{\text {fin }}(\mathbb{H})$, cf. Definition 11 ; $\mathfrak{E}$ is given by $\mathfrak{E}(\mathbb{H})$ and $F$ is given by $\left.F(u)=C_{t_{0}}(u)^{\dagger} C_{t_{0}}(u) \cdot \mathfrak{M}=\mathfrak{E}(\mathbb{H})_{\mathfrak{T}}\right]$.

- $\mathcal{U}$ is a partially ordered set with unit history 1 and null history 0 .

- $\mathcal{U}$ is a partial semigroup with composition law o, cf. (Isham, 1994). $a \circ b$ is welldefined if $t_{f}(a)<t_{i}(b)$. In this case we say that $a$ PROCEEDS $b$ or that $b$ FOLLOWs $a$. Further, $1 \circ a=a \circ 1=a$ and $a \circ 0=0 \circ a=0$. If $a \circ b$ is defined, then $a \circ b=a \wedge b$, in particular the right hand side is well-defined.

- The partial ordering on $\mathcal{U}$ induces a partial unary operation $\neg$ (complementation) and two partial binary operations $\wedge$ and $\vee$ (meet and join) on $\mathcal{U}$.

2. The space of decoherence functionals.

A decoherence functional is a map $d: \mathfrak{M} \times \mathfrak{M} \rightarrow \mathbb{C}$ which satisfies for all $\alpha, \alpha^{\prime}, \beta \in \mathfrak{M}$

- $d(\alpha, \alpha) \in \mathbb{R}$ and $d(\alpha, \alpha) \geq 0$.

- $d(\alpha, \beta)=d(\beta, \alpha)^{*}$.

- $d(1,1)=1$.

- $d(0, \alpha)=0$, for all $\alpha$.

- $d\left(\alpha_{1} \oplus \alpha_{2}, \beta\right)=d\left(\alpha_{1}, \beta\right)+d\left(\alpha_{2}, \beta\right)$ for all $\alpha_{1}, \alpha_{2}, \beta \in \mathfrak{M}$ for which $\alpha_{1} \oplus \alpha_{2}$ is welldefined.

3. The space $\mathfrak{U}$ of general history propositions.

- $\mathfrak{U}$ is the quotient space of the free lattice generated by $\mathcal{U}$ by the congruence relation induced by the partial ordering on $\mathcal{U}$ [see Remark 10].

- There exists an embedding $\tau: \mathcal{U} \rightarrow \mathfrak{U}$, i.e., $\tau(\mathcal{U}) \subset \mathfrak{U}$. 
4. The physical interpretation.

- $\mathfrak{U}$ cannot globally be mapped to $\mathcal{U}$ or to $\mathfrak{M}$ respectively. The physically interesting subsets of $\mathfrak{U}$ are the 'allowed' Boolean sublattices $\mathfrak{U}_{0}$ of $\mathfrak{U}$ (see Definition 23) on which the canonical map $\mathfrak{N}$ can be uniquely extended to a valuation $\mathrm{B}$ on $\mathfrak{U}_{0}$ with values in $\mathfrak{M}$ such that for every $u \in \mathfrak{U}_{0}^{\prime}$ the value $\mathrm{B}(u)$ of this extension does not depend upon the particular 'allowed' Boolean lattice $\mathfrak{U}_{0}^{\prime}$ chosen.

- The decoherence functional induces a probability measure on the consistent (w.r.t. the decoherence functional) 'allowed' Boolean sublattices of $\mathfrak{U}$.

- On the 'allowed' Boolean sublattices of $\mathfrak{U}$ the decoherence functional defines a partial logical implication which allows to make logical inferences.

- The D-poset $(\mathfrak{M}, \oplus)$ may be not unique. There may be other D-posets $\left(\mathfrak{M}^{\prime}, \oplus^{\prime}\right)$ containing $\mathfrak{N}(\mathcal{U})$ such that the decoherence functionals can be extended to $\oplus^{\prime}$-additive functionals on $\mathfrak{M}^{\prime} \times \mathfrak{M}^{\prime}$. It is possible to define 'allowed' Boolean sublattices $\mathfrak{U}_{0}^{\prime}$ of $\mathfrak{U}$ with respect to $\mathfrak{M}^{\prime}$. The decoherence functional induces a probability measure on the consistent 'allowed' Boolean sublattices $\mathfrak{U}_{0}^{\prime}$ of $\mathfrak{U}$. Moreover, the decoherence functional defines a partial logical implication on the Boolean sublattices $\mathfrak{U}_{0}^{\prime}$ of $\mathfrak{U}$.

- there exist D-posets $\mathcal{E U}$ of effect histories isomorphic to $\mathfrak{E}$ [e.g., the full D-poset of effect histories] on which every decoherence functional induces a probability measure, i.e., for which there exists a D-poset isomorphism $\mathfrak{I}: \mathcal{E U} \rightarrow \mathfrak{E} \subset \mathfrak{N}(\mathcal{U})$ mapping the space $\mathcal{E U}$ bijectively to the D-poset $\mathfrak{E}$ such that the decoherence functional can be extended to $\mathfrak{I}(\mathcal{E U})$ and such that

$$
d(\mathfrak{I}(a) \oplus \mathfrak{I}(b), \mathfrak{I}(a) \oplus \mathfrak{I}(b))=d(\mathfrak{I}(a), \mathfrak{I}(a))+d(\mathfrak{I}(b), \mathfrak{I}(b)), \text { for all } a, b \in \mathcal{E U},
$$

whenever the left hand side is well-defined. In particular, no consistency condition is required. Under certain additional conditions it is also possible to define an unambiguous partial implication on $\mathcal{E U}$.

- All probability measures defined on some consistent allowed Boolean sublattices of $\mathfrak{U}$ defined with respect to some of the various D-posets $\mathfrak{M}, \mathfrak{M}^{\prime}$ or on the D-posets of histories $\mathcal{E U}$ have equal physical status in the theory and have to be treated egalitarianly. There seems to be no reason to prefer one class over the others.

\section{Discussion and Conclusion}

Above, we have defined the decoherence functional $\widehat{d}_{\varrho, S}$ on pairs of homogeneous elements in $\left(\mathfrak{E}(\mathbb{H})_{t_{1}} \otimes_{\mathbb{N}} \cdots \otimes_{\mathbb{N}} \mathfrak{E}(\mathbb{H})_{t_{n}}\right) \times\left(\mathfrak{E}(\mathbb{H})_{t_{1}} \otimes_{\mathbb{N}} \cdots \otimes_{\mathbb{N}} \mathfrak{E}(\mathbb{H})_{t_{n}}\right)$ by

$$
\widehat{d}_{\varrho, S}(a, b):=\operatorname{tr}\left(C_{t_{0}}^{\prime}(\sqrt{a}) \varrho C_{t_{0}}^{\prime}(\sqrt{b})^{\dagger}\right),
$$

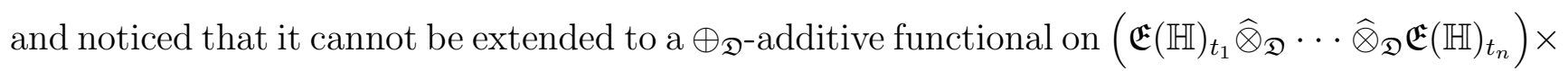


$\times\left(\mathfrak{E}(\mathbb{H})_{t_{1}} \widehat{\otimes}_{\mathfrak{D}} \cdots \widehat{\otimes}_{\mathfrak{D}} \mathfrak{E}(\mathbb{H})_{t_{n}}\right)$. However, if we define a decoherence functional $\tilde{d}_{\varrho, S}$ on pairs of square roots of homogeneous elements in $\left(\mathfrak{E}(\mathbb{H})_{t_{1}} \otimes_{\mathbb{N}} \cdots \otimes_{\mathbb{N}} \mathfrak{E}(\mathbb{H})_{t_{n}}\right) \times\left(\mathfrak{E}(\mathbb{H})_{t_{1}} \otimes_{\mathbb{N}} \cdots \otimes_{\mathbb{N}} \mathfrak{E}(\mathbb{H})_{t_{n}}\right)$ by

$$
\widetilde{d}_{\varrho, S}(\sqrt{a}, \sqrt{b}):=\operatorname{tr}\left(C_{t_{0}}^{\prime}(\sqrt{a}) \varrho C_{t_{0}}^{\prime}(\sqrt{b})^{\dagger}\right)
$$

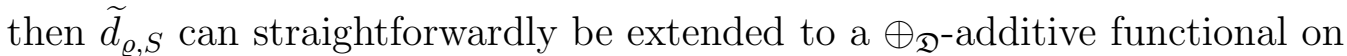
$\left(\mathfrak{E}(\mathbb{H})_{t_{1}} \widehat{\otimes}_{\mathfrak{D}} \cdots \widehat{\otimes}_{\mathfrak{D}} \mathfrak{E}(\mathbb{H})_{t_{n}}\right) \times\left(\mathfrak{E}(\mathbb{H})_{t_{1}} \widehat{\otimes}_{\mathfrak{D}} \cdots \widehat{\otimes}_{\mathfrak{D}} \mathfrak{E}(\mathbb{H})_{t_{n}}\right)$. However, this approach and the one discussed in Section 4.3 are mathematically equivalent since $\sqrt{a} \oplus_{\mathfrak{D}} \sqrt{b}$ is well-defined if and only if $\sqrt{a \widetilde{\oplus}_{\mathfrak{D}} b}$ is well-defined for arbitrary $a, b \in \mathfrak{E}(\mathbb{H})_{t_{1}} \widetilde{\otimes}_{\mathfrak{D}} \cdots \widetilde{\otimes}_{\mathfrak{D}} \mathfrak{E}(\mathbb{H})_{t_{n}}$.

We add a few remarks about the physical significance of the sets of effect histories of order $k>0$. In such effect histories the physical interesting physical qualities are always repeated at $k$ successive times. If we restrict ourselves to ordinary physical qualities represented by projection operators, then repetition of some physical quality adds nothing new. This fact is mathematically expressed through the equation $P_{1} \oplus_{\alpha} P_{2}=P_{1} \oplus P_{2}$ for all $\alpha \in \mathbb{Q}, \alpha>0$ and all projection operators $P_{1}$ and $P_{2}$ for which $P_{1} \oplus P_{2}$ is well-defined. Hence, for histories of ordinary physical qualities all different D-poset structures coincide. The situation that for effect histories there are different inequivalent algebraic structures (D-poset structures) which have to be dealt with on the same footing is a new aspect of our generalized history approach.

We have already mentioned above that the set of effects does not fulfil some requirements which are habitually associated with the notion of property. In particular effects can in general neither be measured ideally nor repeatedly. Moreover, in general the effect $1-F$ cannot be interpreted as the property complementary to the property represented by the effect $F$. The same is true for, e.g., $\left(1-F^{1 / 2}\right)^{2}$. Of course, in mathematical terms $1-F$ and $\left(1-F^{1 / 2}\right)^{2}$ simply are the complements of $F$ in the D-posets $(\mathfrak{E}(\mathbb{H}), \oplus)$ or $(\mathfrak{E}(\mathbb{H}), \widetilde{\oplus})$ respectively. However, if in a measurement of the effect $F$ the measuring apparatus intended to measure $F$ is not triggered, then we can say that the effect $F$ has not occurred (trivial) but in general we cannot say that there is another complementary effect which has occurred instead. This is in particular true for effects $F$ satisfying $F \leq \frac{1}{2}$ or $\frac{1}{2} \leq F$. Therefore some authors claim that only regular effects whose spectrum extends both below as well as above the value $\frac{1}{2}$ represent the (unsharp) properties of a quantum mechanical system (Busch et al., 1995). However, in the present work all effects are treated on the same footing and no ad hoc assumptions are added to single out effects representing the 'well-behaved' properties of a quantum mechanical system.

In the literature of the standard consistent histories approach some authors find it necessary to consider various approximate consistency conditions in order to describe the classical or the quasiclassical properties of quantum mechanical systems. For instance, Omnès associates 'quasi-projectors' with macroscopic regular cells in classical phase space. However, strictly speaking histories involving quasi-projectors lie outside the mathematical framework of the standard consistent histories approach and it is a little bit disconcerting that the discussion of the (semi)classical limit of quantum mechanics in the consistent histories approach involves 
approximations of the fundamental concepts of the theory, even when it is of "no practical importance.' This point may seem a bit pedantic at first, but this author nevertheless believes that it is also a quite unsatisfactory state of affairs in a physical theory. The solution of this problem should be clear now. Of course, quasi-projectors are only a special sort of effects. Thus histories of quasi-projectors fit perfectly well in our generalized history theory and no ad hoc modifications of the basic concepts and principles are necessary in the discussion of the (semi)classical limit of quantum mechanics.

Gell-Mann and Hartle encounter in their discussions of quasiclassical domains and measurement processes the situation that the consistency conditions and the probability sum rules for physically interesting histories are only approximately satisfied (see in particular section II.11 in (Hartle, 1991)). The standard 'for all practical purposes' argument to justify the use of approximate probabilities and approximate consistency conditions is that if the violation is small enough, then no experiment could detect the discrepancy. Dowker and Kent (1996) stress that 'this seems a rather casual disruption of the mathematical structure of a fundamental theory.' Indeed, this question is not a question of practicability but rather a question of principle. While admittedly approximations of any (reasonable) kind may be used for the computation of the (numerical) predictions of a theory, the basic principles and concepts of a theory should be formulated without invoking any kind of approximation. Otherwise the result would be a theory with semantically slippery rules of interpretation or without firm mathematical foundations. In any case our generalized history approach allows to incorporate any kind of 'unsharp' physical quality into the theory at a fundamental level, regardless whether it describes the quasiclassical domain of the universe or a measurement situation with limited resolution or other. Histories of generalized physical qualities and effect histories have a clear-cut status in the theory and thus concepts of approximate consistency are superfluous. Since these questions are not the main topic of the present work, we postpone a thorough discussion to the future.

The problem of interpreting a physical theory is of foremost importance in the physical science but especially in the case of quantum theory difficult. The axioms of every interpretation of a physical theory are always introduced by fiat. They are independent of and cannot be derived from the formalism. Thus it comes as no surprise that no agreement about the interpretation of quantum mechanics has been achieved yet. Nevertheless even nowadays most physicists do not care too much about interpretational issues and simply rely on one of the diverse versions of the so-called Copenhagen interpretation of quantum mechanics. This interpretation is (as is well-known) plagued with paradoxes and puzzles such as the subject-object muddle and the actualization of facts as a consequence of our measurements. One of its basic claims is that main aspects of the microscopic world are in principle unanalyzable. Furthermore, it has been stressed by Omnès (1992) that the Copenhagen interpretation is incomplete. The paradoxes of the Copenhagen interpretation of quantum mechanics cannot be resolved but only be removed by another interpretation of quantum mechanics.

In the last decades there have been several attempts to construct a realistic and individual interpretation of quantum mechanics. In this work we studied two such attempts, namely the generalized operational quantum theory and the logical interpretation based on the concept of 
histories. We investigated their interrelationship and constructed a generalized logical interpretation. Both the generalized operational interpretation and the old logical interpretation can be viewed as special cases of our generalized logical interpretation. Our generalized Rule 2 is, however, only a first tentative step towards a consistent quantum reasoning involving generalized properties. Unfortunately, there is no general theory of coexistent sets of effects or generalized observables in quantum mechanics. With such a theory it would presumably be possible to considerably improve and generalize our approach and find a more natural definition of the notion of allowed Boolean lattice of effect histories than the one given in Definition 23.

The interpretation of the consistent histories formalism underlying the present work differs significantly from the wide-spread interpretation succinctly summarized under the name $U n$ known Set Interpretation by Kent (1995). Firstly - and perhaps most importantly - we do not claim that nonrelativistic Hamiltonian quantum mechanics can be applied to the "whole universe,' see Note 5. Hence, we also do not adopt the view that exactly one history is realized and describes all of physics. Whether or not the classical and quasiclassical features of the observable world can be fully understood in the consistent histories formalism is an open problem. The most promising and most concrete description of the semiclassical limit of quantum mechanics in the framework of consistent histories has been given by Omnès (1994, Chapter 6). Secondly, we assert that different consistent sets of histories are complementary in the sense described above and should be treated on the same footing (this is completely analogous to the assertion that position and momentum are complementary variables in the description of an elementary particle). Quantum theory ascribes probabilities to different possible events. The propensity that some particular event occurs depends upon the quantum system itself and upon the integral physical situation. Particularly, in measurement situations the result of the measurement depends upon the object under study and upon the mode of observation. At present no rule determining a preferred consistent set of histories for the universe (the Unknown Set) is known; in our interpretation such a rule will hardly be needed: instead for every quantum system and every 'physical situation' one needs a rule fixing the 'correct' consistent set. In measurement situations the choice of the consistent set is determined by the measuring apparatuses.

There are three methodical arguments supporting the logical interpretation. Since according to the logical rule any allowed reasoning relates only properties belonging to some 'allowed' Boolean algebra and since there can as a matter of principle never arise a paradoxical or inconsistent situation in a Boolean logic, all paradoxes and inconsistencies have been expelled from the language of quantum mechanics by one single simple rule. Thus the three arguments supporting the logical interpretation are in brief

- Nonrelativistic quantum mechanics with the generalized logical interpretation (and together with decoherence) gives unambiguous predictions for every conceivable experimental situation in nonrelativistic quantum mechanics;

- Simplicity and economy of principles;

- The logical interpretation is free of logical paradoxes. 
Examples illustrating the third point have been discussed by Omnès (1994). However, at least one mystery has remained. The microscopic world is unanalyzable. Quantum physics as viewed by the logical interpretation provides no model for what 'actually happens.'

\section{Acknowledgments}

The author wants to thank Frank Steiner for encouraging and supporting this work and Christian Grosche for drawing his attention upon the work of Karl Popper. Financial support given by Deutsche Forschungsgemeinschaft (Graduiertenkolleg für theoretische Elementarteilchenphysik) is gratefully acknowledged. 


\section{References}

J.S. Bell (1987). Speakable and Unspeakable in Quantum Mechanics, Cambridge University Press.

E.G. Beltrametti and G. Cassinelli (1981). The Logic of Quantum Mechanics, Addison-Wesley, Reading.

G. Birkhoff (1967). Lattice Theory, American Mathematical Society Colloquium Publications, 25, 3rd Edition.

G. Birkhoff and J. von Neumann (1936). Annals of Mathematics, 37, 823.

P. Busch, M. Grabowski and P.J. Lahti (1989). Foundations of Physics Letters, 2, 331.

P. Busch, P.J. Lahti and P. Mittelstaedt (1991). The Quantum Theory of Measurement, Lecture Notes in Physics m2, Springer Verlag, Berlin.

P. Busch, M. Grabowski and P.J. Lahti (1995). Operational Quantum Physics, Lecture Notes in Physics m31, Springer Verlag, Berlin.

G. Cattaneo and F. Laudisa (1994). Foundations of Physics, 24, 631.

E.B. Davies (1976). Quantum Theory of Open Systems, Academic Press, London.

F. Dowker and A. Kent (1996). Journal of Statistical Physics, 82, 1575.

A. Dvurečenskij (1995). Transactions of the American Mathematical Society, 347, 1043.

A. Dvurečenskij and S. Pulmannová (1994a). International Journal of Theoretical Physics, 33, 819.

A. Dvurečenskij and S. Pulmannová (1994b). Reports on Mathematical Physics, 34, 151.

A. Dvurečenskij and S. Pulmannová (1994c). Reports on Mathematical Physics, 34, 251.

J. Finkelstein (1993). Physical Review D, 47, 5430.

D.J. Foulis and M.K. Bennett (1994). Foundations of Physics, 24, 1331.

M. Gell-Mann and J.B. Hartle (1990a). in: Proceedings of the 25th International Conference on High Energy Physics, Singapore, August 2-8, 1990, 1303, K.K. Phua and Y. Yamaguchi, (eds.), World Scientific, Singapore.

M. Gell-Mann and J.B. Hartle (1990b). in: Proceedings of the Third International Symposium on the Foundations of Quantum Mechanics in the Light of New Technology, 321, S. Kobayashi, H. Ezawa, Y. Murayama and S. Nomura (eds.), Physical Society of Japan, Tokyo.

M. Gell-Mann and J.B. Hartle (1990c). in: Complexity, Entropy and the Physics of Information, Santa Fe Institute Studies in the Science of Complexity, Vol. VIII, 425, W. Zurek (ed.), Addison-Wesley, Reading. 
M. Gell-Mann and J.B. Hartle (1993). Physical Review D, 47, 3345.

M. Gell-Mann and J.B. Hartle (1994). Equivalent Sets of Histories and Multiple Quasiclassical Domains, preprint, gr-qc/9404013.

M. Gell-Mann and J.B. Hartle (1995). Strong Decoherence, preprint, gr-qc/9509054.

R. Giuntini and H. Greuling (1989). Foundations of Physics, 19, 931.

A.M. Gleason (1957). Journal of Mathematics and Mechanics, 6, 885.

S. Goldstein and D. Page (1995). Physical Review Letters, 74, 3715.

R.B. Griffiths (1984). Journal of Statistical Physics, 36, 219.

R.B. Griffiths (1995). Consistent Quantum Reasoning, preprint, quant-ph/9505009.

J.B. Hartle (1991). in: Quantum Cosmology and Baby Universes: Proceedings of the 1989 Jerusalem Winter School for Theoretical Physics, 65, S. Coleman, J.B. Hartle, T. Piran and S. Weinberg (eds.), World Scientific, Singapore.

J.B. Hartle (1994). in: Proceedings of the 1992 Les Houches Summer School, B. Julia and J. Zinn-Justin (eds.), Les Houches Summer School Proceedings Vol. LVII, North Holland, Amsterdam.

C.J. Isham (1994). Journal of Mathematical Physics, 35, 2157.

C.J. Isham and N. Linden (1994). Journal of Mathematical Physics, 35, 5452.

M. Jammer (1974). The Philosophy of Quantum Mechanics, J. Wiley, New York.

J.M. Jauch (1968). Foundations of Quantum Mechanics, Addison-Wesley.

A. Kent (1995). Remarks on Consistent Histories and Bohmian Mechanics, to appear in: Bohmian Mechanics and Quantum Theory: An Appraisal, J. Cushing, A. Fine and S. Goldstein (eds.), Kluwer Academic Press; quant-ph/9511032.

F. Kôpka (1992). Tatra Mountains Mathematical Publications, 1, 83.

F. Kôpka and F. Chovanec (1994). Mathematica Slovaca, 44, 21.

K. Kraus (1983). States, Effects, and Operations, Lecture Notes in Physics vol. 190, Springer Verlag, Berlin.

A. Landé (1965). New Foundations of Quantum Mechanics, Cambridge University Press.

H. Langer (1962). Acta Mathematica Academiae Scientiarum Hungaricae, 13, 415.

G. Ludwig (1970). Deutung des Begriffs "physikalische Theorie" und axiomatische Grundlegung der Hilbertraumstruktur der Quantenmechanik durch Hauptsätze des Messens, Lecture Notes in Physics, 4, Springer Verlag, Berlin.

G. Ludwig (1972-79). Einführung in die Grundlagen der Theoretischen Physik, Volumes 1-4, Vieweg, Braunschweig.

G. Ludwig (1983). Foundations of Quantum Mechanics I, Springer Verlag, Berlin.

S. Maeda (1989). Reviews in Mathematical Physics, 1, 235. 
J. von Neumann (1932). Mathematische Grundlagen der Quantenmechanik, Springer Verlag, Berlin.

R. Omnès (1988a). Journal of Statistical Physics, 53, 893.

R. Omnès (1988b). Journal of Statistical Physics, 53, 933.

R. Omnès (1988c). Journal of Statistical Physics, 53, 957.

R. Omnès (1989). Journal of Statistical Physics, 57, 357.

R. Omnès (1990). Annals of Physics (N.Y.), 201, 354.

R. Omnès (1991). Journal of Statistical Physics, 62, 841.

R. Omnès (1992). Reviews of Modern Physics, 64, 339.

R. Omnès (1994). The Interpretation of Quantum Mechanics, Princeton University Press.

R. Omnès (1995). Foundations of Physics, 25, 605.

M. Ozawa (1984). Journal of Mathematical Physics, 25, 79.

K.R. Popper (1959/60). The British Journal for the Philosophy of Science, 10, 25.

K.R. Popper (1982). Quantum Theory and the Schism in Physics, Rowman and Littlefield, Totowa.

K.R. Popper (1994). Logik der Forschung, 10. Auflage, J.C.B. Mohr, Tübingen.

H. Primas (1983). Chemistry, Quantum Mechanics and Reductionism, 2nd Edition, Springer Verlag, Berlin.

E. Prugověcki (1992). Quantum Geometry, a Framework for Quantum General Relativity, Kluwer Academic, Dordrecht.

S. Pulmannová (1995). International Journal of Theoretical Physics, 34, 189.

E. Scheibe (1973). The Logical Analysis of Quantum Mechanics, Pergamon Press, Oxford.

L.A. Skornjakov (1977). Elements of Lattice Theory, Adam Hilger, Bristol.

H.D. Zeh (n.d.). The Program of Decoherence: Ideas and Concepts, intended as Chapter II in: D. Guilini, E. Joos, C. Kiefer, J. Kupsch, I.-O. Stamatescu and H.D. Zeh, Decoherence and the Emergence of a Classical World, Springer Verlag, Berlin, in preparation; also quant-ph/9506020. 